\title{
Application of a High Q, Low Cost Hemispherical Cavity Resonator to Microwave Oscillators
}

by

\section{Elizabeth Ruscito, B.Sc.}

\author{
A thesis submitted to the \\ Faculty of Graduate and Postdoctoral Affairs \\ in partial fulfilment of \\ the requirements for the degree of

\section{Master of Applied Science}

in

Electrical and Computer Engineering

Ottawa-Carleton Institute for Electrical and Computer Engineering

Department of Electronics

Faculty of Engineering

Carleton University

Ottawa, Ontario, Canada

September 2011

Copyright $\mathbb{C}$ Elizabeth Ruscito 2011 


$\begin{array}{ll}\begin{array}{l}\text { Library and Archives } \\ \text { Canada }\end{array} & \begin{array}{l}\text { Bibliotheque et } \\ \text { Archives Canada }\end{array} \\ \begin{array}{l}\text { Published Heritage } \\ \text { Branch }\end{array} & \begin{array}{l}\text { Direction du } \\ \text { Patrimoine de l'édition }\end{array} \\ \begin{array}{l}\text { 395 Wellington Street } \\ \text { Ottawa ON K1A ON4 } \\ \text { Canada }\end{array} & \begin{array}{l}\text { 395, rue Wellington } \\ \text { Ottawa ON K1A ON4 } \\ \text { Canada }\end{array}\end{array}$

Your file Votre référence

ISBN: 978-0-494-83050-5

Our file Notre référence

ISBN: 978-0-494-83050-5

NOTICE:

The author has granted a nonexclusive license allowing Library and Archives Canada to reproduce, publish, archive, preserve, conserve, communicate to the public by telecommunication or on the Internet, loan, distribute and sell theses worldwide, for commercial or noncommercial purposes, in microform, paper, electronic and/or any other formats.

The author retains copyright ownership and moral rights in this thesis. Neither the thesis nor substantial extracts from it may be printed or otherwise reproduced without the author's permission.
AVIS:

L'auteur a accordé une licence non exclusive permettant à la Bibliothèque et Archives Canada de reproduire, publier, archiver, sauvegarder, conserver, transmettre au public par télécommunication ou par l'Internet, prêter, distribuer et vendre des thèses partout dans le monde, à des fins commerciales ou autres, sur support microforme, papier, électronique et/ou autres formats.

L'auteur conserve la propriété du droit d'auteur et des droits moraux qui protège cette thèse. $\mathrm{Ni}$ la thèse ni des extraits substantiels de celle-ci ne doivent être imprimés ou autrement reproduits sans son autorisation.
In compliance with the Canadian Privacy Act some supporting forms may have been removed from this thesis.

While these forms may be included in the document page count, their removal does not represent any loss of content from the thesis.
Conformément à la loi canadienne sur la protection de la vie privée, quelques formulaires secondaires ont été enlevés de cette thèse.

Bien que ces formulaires aient inclus dans la pagination, il n'y aura aucun contenu manquant.

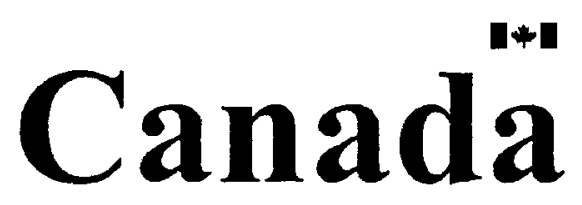




\section{Abstract}

This research project presents the design, simulation, fabrication and assessment of a low-cost high quality factor hemispherical cavity resonator for sub harmonic E-band applications. The hemispherical cavity was embedded in a brass package by commercial machining techniques and feeding structures were implemented on a low loss millimeter wave substrate, RT/Duroid 5880 .

Aperture coupling theory was used to design the cavity and simple electromagnetic equations were used to calculate the resonant frequency, loaded and unloaded quality factors. 3D finite element simulations from HFSS were performed on the cavity design and a sensitivity analysis was completed on the parameters affecting the loaded and unloaded quality factors.

The hemispherical cavity was fabricated and measured for comparisons with simulated results. A resonant frequency of $19.96 \mathrm{GHz}$ was measured and the highest achieved is unloaded quality factor of 2565 along with a loaded quality factor of 2532 . 


\section{Acknowledgements}

This research project would not have been possible without the continuous support from my supervisors Prof. Barry Syrett and Prof. Langis Roy. Through their guidance I have learned to challenge myself and I am grateful for the opportunity they have given me. Also, many thanks to Prof. Rony E. Amaya, without whose precious input this project would not have been possible. Thanks to Adrian Momciu for taking the time to help with the prototype circuits and with measurements and finally thanks to DragonWave inc. and the Communications Research Centre (CRC) for giving me the possibility to pursue this project.

Special thanks to Hedy Chuang, my partner in crime through these years. 能夠一起生存在這精神病院裡, 真的很好玩。自行簽入, 簽出受控。.

Un ringraziamento particolare al mio ragazzo Alessandro, che mi ha saputo sostenere anche da cosi lontano. Grazie per le lunghe chattate...lo sò che questi due anni sono stati duri ma ce l'abbiamo fatta...

Finalmente, un GRAZIE a Papà e Mamma. Sembra banale ma senza la vostra spinta e il vostro continuo sostegno non avrei ottenuto questa seconda laurea, sono "dottoressa" soltanto grazie a voi. Thanks to Fran, without our shopping sprees I would not have survived, Anna thanks to your Sunday gnocchi I was able to recharge for the week, and Gio, I will miss the hours of math tutoring...make me proud! Vi voglio bene!! 


\section{Table of Contents}

$\begin{array}{ll}\text { Abstract } & \text { i }\end{array}$

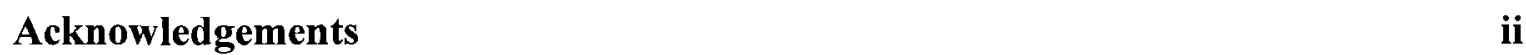

Table of Contents iii

$\begin{array}{lll}\text { List of Tables } & \text { vi }\end{array}$

$\begin{array}{ll}\text { List of Figures } & \text { vii }\end{array}$

$\begin{array}{ll}\text { List of Abbreviations and Symbols } & \mathbf{x}\end{array}$

$\begin{array}{ll}\text { Chapter } 1 & 1\end{array}$

$\begin{array}{ll}\text { Introduction } & 1\end{array}$

1.1 Motivation and Overview ................................................................. 1

1.2 Thesis Objectives, Contributions and System Concept .............................. 4

1.3 Thesis Organization ........................................................................ 7

$\begin{array}{lr}\text { Chapter } 2 & 8\end{array}$

$\begin{array}{lr}\text { Microwave Resonators } & 8\end{array}$

2.1 Basic Background Theory ..................................................................... 8

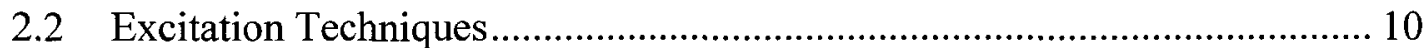

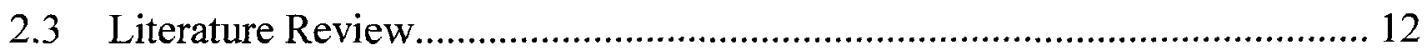

$\begin{array}{ll}\text { Chapter } 3 & 15\end{array}$

$\begin{array}{ll}\text { Cavity Resonator Design } & 15\end{array}$

3.1 The Unloaded Hemispherical Cavity Resonator...................................... 15 
3.2 Resonant Frequency and Cavity Dimensions ………................................... 18

3.3 The Unloaded Quality Factor......................................................................... 19

3.4 The Loaded Quality Factor ........................................................................ 24

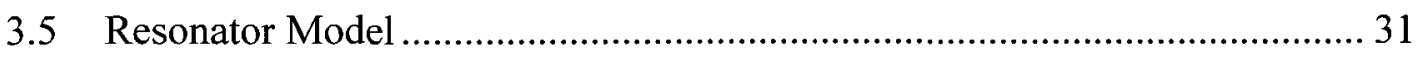

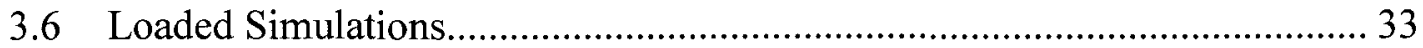

$\begin{array}{lr}\text { Chapter } 4 & 38\end{array}$

$\begin{array}{lr}\text { Resonator Study } & 38\end{array}$

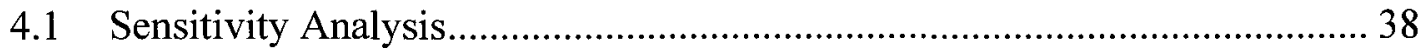

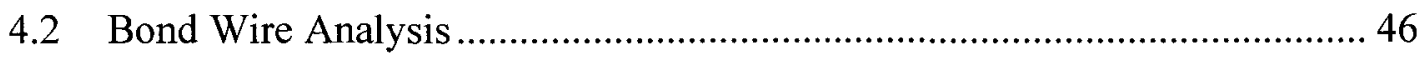

4.3 RT/duroid® 5880 Microstrip Resonator ....................................................... 52

4.4 Manufacturing Issues of the Resonator Package ........................................... 53

4.5 A Tunable Hemispherical Cavity Resonator ……………………….............. 58

$\begin{array}{lr}\text { Chapter } 5 & 62\end{array}$

Measurements and Performance of the Hemispherical Cavity Resonator 62

5.1 Resonator Fabrication and Dimensional Tolerances ................................... 62

5.1.1 RT/Duroid 5880 PCB layout issues............................................... 63

5.1.2 Hemispherical Cavity machining..................................................... 66

5.1.3 Complete Assembly ........................................................................ 67

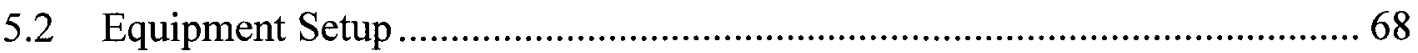

5.3 Performance and measurements of the hemispherical cavity resonator ........ 69

5.4 Discussion of Results and Sources of Error..................................................... 74

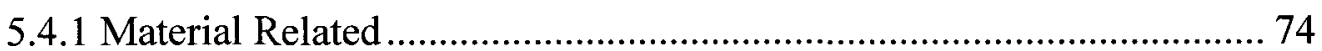

5.4.2 Assembly and Design........................................................................... 74

5.4.3 Process Tolerances ........................................................................ 75

5.4.4 Simulation Assumptions ..................................................................... 75

5.5 E-band Oscillator Phase noise.................................................................... 76

5.6 Manufacturing issues with the tuning capability of the resonator .................. 79

$\begin{array}{lr}\text { Chapter } 6 & 81\end{array}$

$\begin{array}{lr}\text { Conclusions and Future Work } & \mathbf{8 1}\end{array}$ 


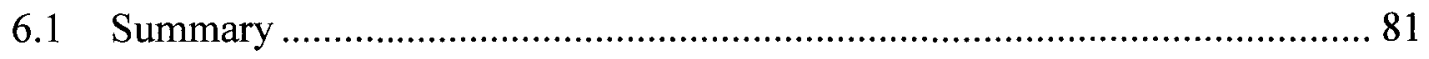

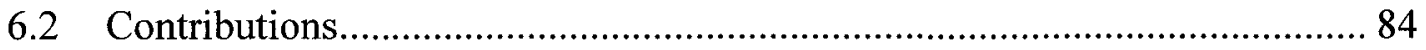

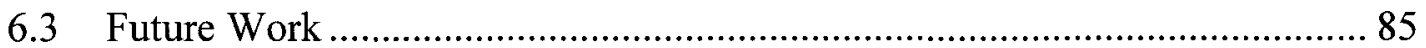

$\begin{array}{ll}\text { Appendix A } & \mathbf{8 7}\end{array}$

$\begin{array}{lr}\text { References } & \mathbf{8 8}\end{array}$ 


\section{List of Tables}

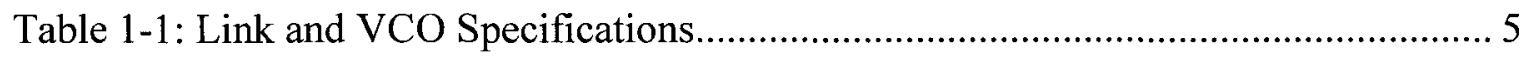

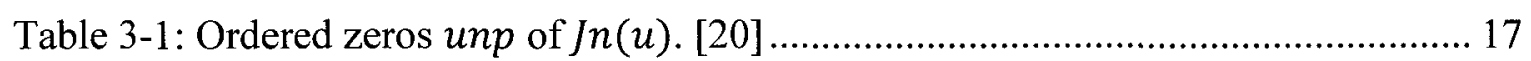

Table 3-2: Ordered zeros $u n p^{\prime}$ of $J n^{\prime}\left(u^{\prime}\right)$. [20] ......................................................... 17

Table 3-3: Eigenmode simulations featuring various modes........................................... 24

Table 3-4: Comparison of millimeter wave materials. ................................................... 32

Table 3-5: Eigenmode unloaded Q factors for different JIG plating materials. ............... 33

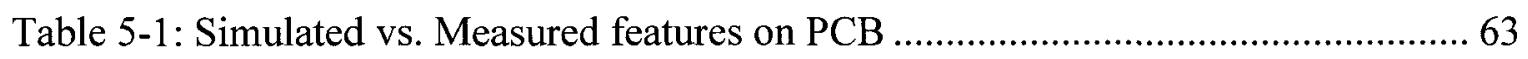

Table 5-2: Simulated and measured quality factors for various aperture radii................. 72

Table 5-3: Review of System Specifications ............................................................... 77

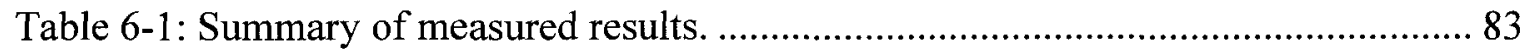

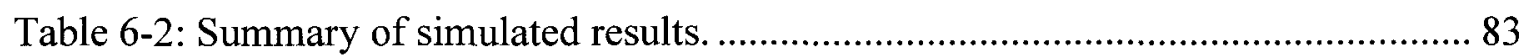




\section{List of Figures}

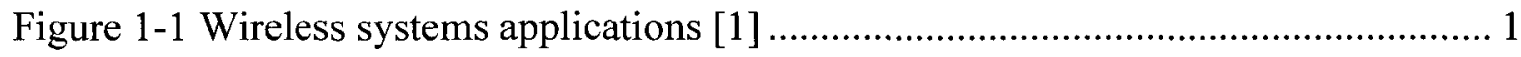

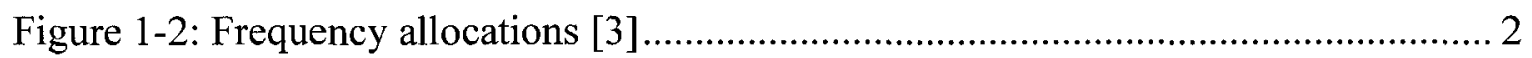

Figure 1-3 Rain attenuation at microwave and millimeter-wave frequencies [3] ............ 3

Figure 1-4: Typical Wireless Transceiver Block Diagram [4] .................................... 3

Figure 1-5: Cross section of proposed oscillator package .........................................6

Figure 2-1: Resonant circuits. (a) Series RLC circuit. (b) Parallel RLC circuit ................ 9

Figure 2-2: Coupling techniques. (a) Microstrip transmission line resonator gap coupled to a microstrip feed line. (b) Rectangular cavity resonator fed by a coaxial probe. (c) Circular cavity resonator aperture coupled to a rectangular waveguide. (d) Dielectric resonator coupled to a microstrip feedline[9] ................................................... 10

Figure 2-3: Classic oscillator phase noise behavior [10] ...................................... 12

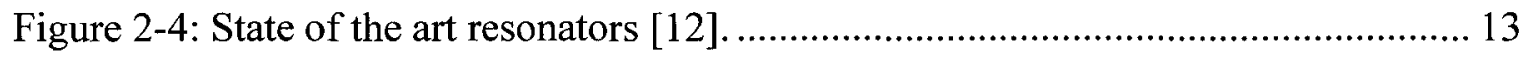

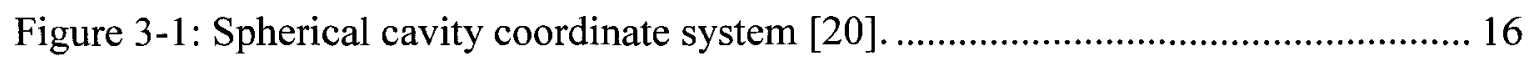

Figure 3-2: Cavity dimensions ........................................................................... 18

Figure 3-3: Electric and Magnetic fields of the perfect hemispherical cavity at the fundamental $\mathrm{TM}_{011}$ mode.................................................................................. 19

Figure 3-4: Electric field mode pattern............................................................ 21

Figure 3-5: Magnetic field mode pattern. ..................................................... 21

Figure 3-6: Skin depth versus Frequency for different materials [21]........................ 23

Figure 3-7: Various shapes of coupling apertures [25] ............................................ 26

Figure 3-8: Parallel Plate waveguide cross section of a microstrip transmission line [28]

Figure 3-9: Aperture coupling between two identical microstrips [28] ....................... 29 
Figure 3-10: Calculated loaded quality factor as a function of aperture radius................ 30

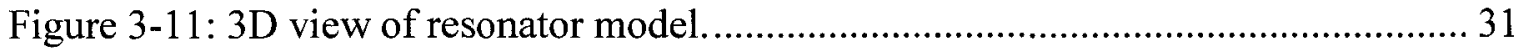

Figure 3-12: Simulated $S_{11}$ of hemispherical cavity resonator for various aperture radii. 34

Figure 3-13: Simulated $S_{11}$ showing undercoupling for hemispherical cavity resonator . 35

Figure 3-14: Calculated $Q_{L}$ vs. Simulated $Q_{L}$ as a function of aperture radius............... 36

Figure 3-15: Wideband spectrum for an aperture radius of $0.6 \mathrm{~mm}$.

Figure 4-1: Return loss vs. frequency for aperture movement in $\pm x$ direction by $\pm 1 \mathrm{mil}$.

Figure 4-2: Movement of aperture in $\pm x$ direction vs. loaded quality factor

Figure 4-3: Return loss vs. frequency for aperture position in $\pm y$ direction by $\pm 0.5 \mathrm{~mm} 42$

Figure 4-4: Position of the aperture in $\pm y$ direction vs. loaded quality factor 42

Figure 4-5: Return loss vs. frequency for variable microstrip line width..... 43

Figure 4-6: Width of microstrip feed line vs. loaded quality factor 44

Figure 4-7: Top view of cavity resonator 44

Figure 4-8: Return loss vs. frequency for variable offset .45

Figure 4-9: Offset vs. loaded quality factor .45

Figure 4-10: Wire bond setup a) 3D view, b) side zoom of wire bond 47

Figure 4-11: Ribbon bond setup a) 3D view, b) side zoom of ribbon bond 48

Figure 4-12: S-parameter data for wire bond. 49

Figure 4-13: Length of wire bond vs. $\left|S_{21}\right|$ 50

Figure 4-14: Wire bond and resonator setup. 51

Figure 4-15: S-parameter data for wire bond and resonator setup, $\mathrm{m} 1$ = with bond wire, $\mathrm{m} 2$ = without bond wire 51

Figure 4-16: Circuit of parallel lumped $\lambda / 4$ short microstrip resonator............................ 52

Figure 4-17: Resonant frequency of $\lambda 4$ short microstrip resonator. .............................. 53

Figure 4-18: Cross section of oscillator package.

Figure 4-19: Gold plated clamp and resonator package …………………….................. 55

Figure 4-20: Modified package for probing of the resonator. .......................................... 55

Figure 4-21: Floor plan of printed circuit board (from simulations) ................................ 57

Figure 4-22: Floor plan of printed circuit board (fabricated), ..........................................5 57

Figure 4-23: Tunable Oscillator Package ………….................................................. 58 
Figure 4-24: The concept behind the tunable cavity resonator.

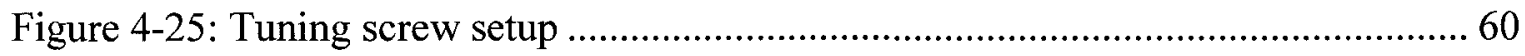

Figure 4-26: Depth of screw vs. Frequency vs. Loaded quality factor...........................60 60

Figure 5-1: Resonator package and PCB complete assembly for measurements............ 64

Figure 5-2: Actual layout of the probe-to-pad transition for de-embedding. .................. 64

Figure 5-3: Zoom of the landing of the probes on the microstrip feed........................65

Figure 5-4: De-embedding the probe-to-pad transition ............................................. 66

Figure 5-5: Top view of complete and assembled package ................................. 67

Figure 5-6: Populated PCB card for final assembly, detail in [7] ...............................6 67

Figure 5-7: 3D view of complete and assembled package. ........................................ 68

Figure 5-8: Measurement equipment and setup....................................................69 69

Figure 5-9: Measured $S_{11}$ of cavity with coupling aperture $0.5 \mathrm{~mm}$ with corresponding

simulated $\mathrm{S}_{11}$

Figure 5-10: Measured $S_{11}$ of cavity resonator with coupling aperture $0.6 \mathrm{~mm}$ with

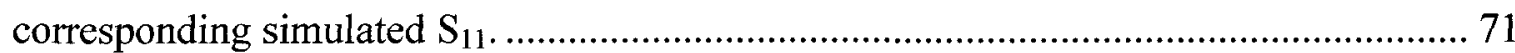

Figure 5-11: Measured versus simulated S-parameter data for various aperture radii.... 72

Figure 5-12: Measured wideband spectrum for an aperture radius of $0.6 \mathrm{~mm}$............... 73

Figure 5-13: Phase noise of the E-band oscillator ............................................. 78

Figure 5-14: Extrapolated phase noise at $100 \mathrm{kHz}$ offset....................................... 79

Figure 5-15: Manufacturing issue of the tunable resonator..................................... 80 


\section{List of Abbreviations and Symbols}

\begin{tabular}{|c|c|}
\hline RF & Radio Frequency \\
\hline $\mathrm{GHz}$ & Giga Hertz \\
\hline $\mathrm{MHz}$ & Mega Hertz \\
\hline Gbps & Giga bit per second \\
\hline LNA & Low Noise Amplifier \\
\hline PA & Power Amplifier \\
\hline IF & Intermediate Frequency \\
\hline COTS & Commercial Off The Shelf \\
\hline AMP & Amplifier \\
\hline $\mathrm{ADC}$ & Analog to Digital Converter \\
\hline DAC & Digital to Analog Converter \\
\hline $\mathrm{TX} / \mathrm{RX}$ & Transmitter/ Receiver \\
\hline $\mathrm{VCO}$ & Voltage Controlled Oscillator \\
\hline$\omega$ & Angular frequency \\
\hline $\mathrm{L}\left(\omega_{\mathrm{m}}\right)$ & Phase Noise \\
\hline $\mathrm{F}$ & Noise Factors \\
\hline $\mathrm{K}$ & Boltzman's constant \\
\hline $\mathrm{T}$ & Absolute Temperature \\
\hline $\mathrm{P}_{\mathrm{S}}$ & Power applied to resonator \\
\hline$\omega_{0}$ & Resonant angular frequency \\
\hline PCB & Printed Circuit Board \\
\hline $\mathrm{G}$ & Active device gain \\
\hline RLC & Resistor/Inductor/Capacitor \\
\hline
\end{tabular}


DRO Dielectric Resonator Oscillator

TE Transverse Electric

TM Transverse Magnetic

VCO Voltage Controlled Oscillator

$\varepsilon \quad$ Permittivity

$\mu \quad$ Permeability

$\varepsilon_{0} \quad$ Vacuum permittivity

$\mu_{\mathrm{o}} \quad$ Vacuum permeability

$\varepsilon_{\mathrm{r}} \quad$ Dielectric constant

$\mathrm{V}_{\mathrm{cav}} \quad$ Cavity effective volume

$V_{\text {ap }} \quad$ Aperture effective volume

$\varepsilon_{\text {eff }} \quad$ Effective permittivity

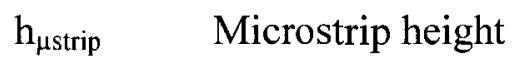

$\mathrm{w}_{\mu s t r i p} \quad$ Microstrip width

K Coupling Coefficient

$\mathrm{Z}_{0} \quad$ Characteristic impedance

$\mathrm{T}$ Transmission Coefficient

$\mathrm{H}_{\mathrm{o}} \quad$ Amplitude of magnetic field

$\lambda_{0} \quad$ Wavelength in free space

LTCC Low-Temperature Co-Fired Ceramic

IC Interconnected Circuits

GaAs Gallium Arsenide

ADS Advanced Design System

DUT Device Under Test

CRC Communication Research Centre 


\section{Chapter 1}

\section{Introduction}

\subsection{Motivation and Overview}

The increasing demand over the years for wireless applications has forced the RF industry to produce high speed, high performance and low cost devices. Wireless systems and communication networks are widely used in all types of industries, Figure 1-1, and are constantly studied and researched.

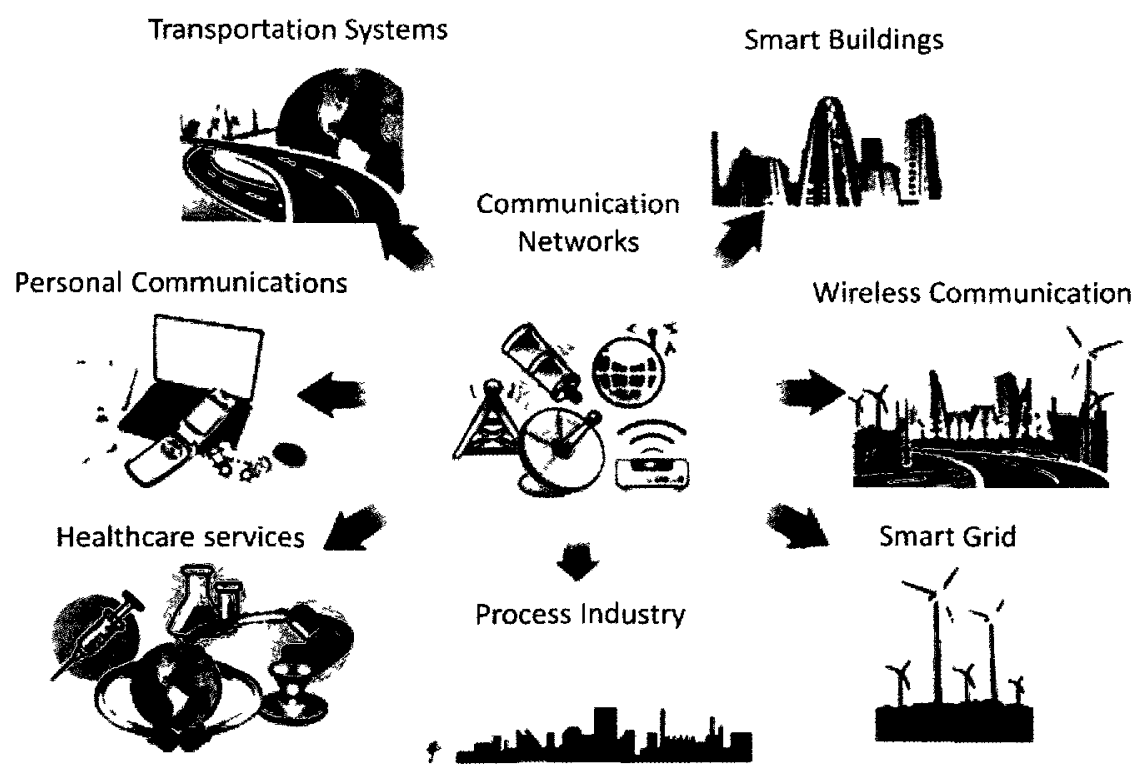

Figure 1-1 Wireless systems applications [1] 
It is the demand for higher bandwidths that has been one of the most challenging aspects in driving the need to improve the performance of microwave components, especially for wireless technologies at E-band frequencies. E-band frequencies cover the $71 \mathrm{GHz}$ to $76 \mathrm{GHz}, 81 \mathrm{GHz}$ to $86 \mathrm{GHz}$ and $92 \mathrm{GHz}$ to $95 \mathrm{GHz}$ bands. These bands are widely used worldwide for ultra high capacity point-to-point communications. The 10 $\mathrm{GHz}$ of bandwidth available in this band, as shown in Figure 1-2, is the most ever allocated by the FCC at any one time [2]. Such bandwidth permits greater data rates which can be accommodated with relatively simple and cost efficient radio transceiver architectures.

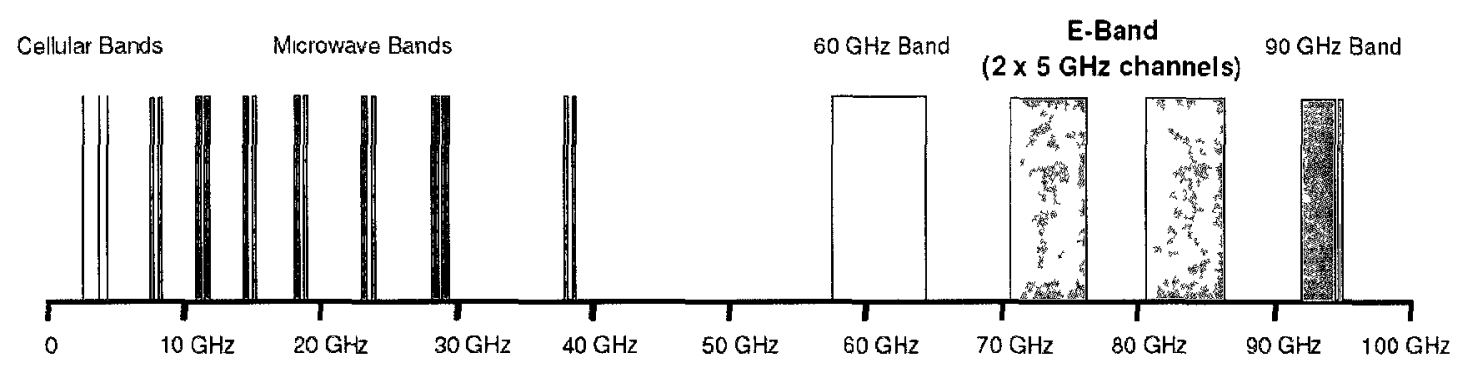

Figure 1-2: Frequency allocations [3]

A major advantage of the E-band allocations is that they are not partitioned into small channels, unlike the lower frequency microwave bandwidths which are sliced into channels of approximately $50 \mathrm{MHz}$ each. This allows the two $5 \mathrm{GHz}$ E-band channels to transmit 100 times more than the largest microwave band. Since there is no need to compress the data into the smaller frequency channels, radio architectures become relatively simple. The E-band wireless systems available offer full-duplex Gigabit Ethernet connectivity at data rates of $1 \mathrm{Gbps}$ and higher in cost effective radio architectures [3]. In addition, apart from covering the longest transmission distances, Eband presents very robust weather resilience as it is not subject to fog, dust or other small particles. It does however present attenuation in the presence of rain as shown in Figure $1-3$. 


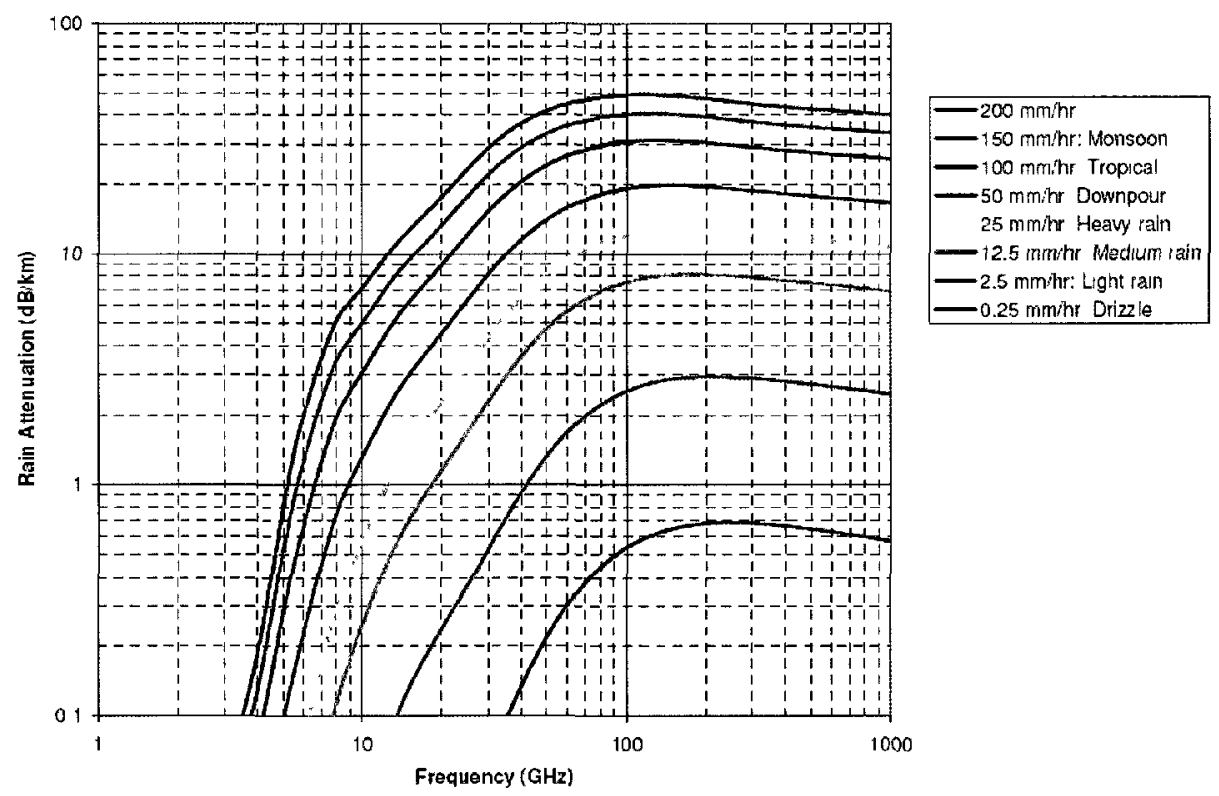

Figure 1-3 Rain attenuation at microwave and millimeter-wave frequencies [3]

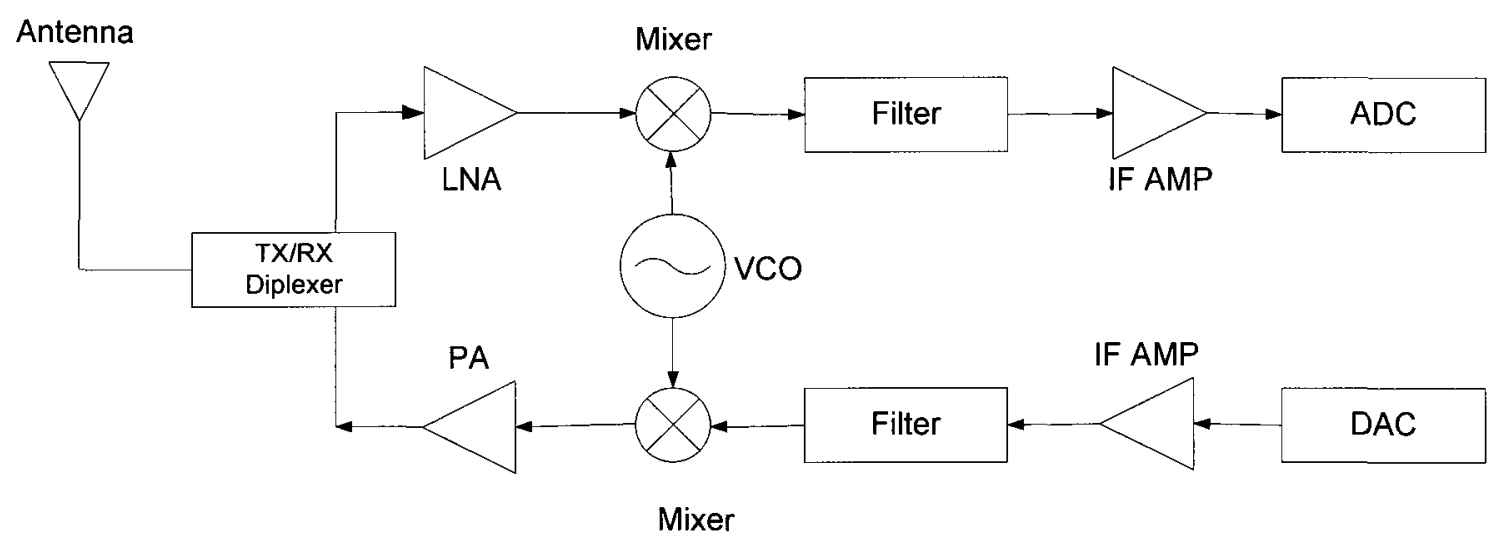

Figure 1-4: Typical Wireless Transceiver Block Diagram [4]

The system level overview of a typical wireless transceiver is shown in Figure 1-4. Voltage Controlled Oscillators (VCOs) are one of the most important components in these radio transceiver architectures. They are used in almost any commercially available device and are used in all radio frequency and wireless systems as shown above. The oscillator is usually the most difficult component to design and in many cases its performance determines the overall characteristics of the system. 
In fact, the innate instability of oscillators manifests itself in a phenomenon called phase noise and it is one of the major issues for RF designers. It degrades the overall performance and distorts or corrupts incoming and outgoing information in a transceiver. In addition, it increases bit error rate in phase modulated applications [5]. Phase noise can be expressed as the frequency range in which the oscillator presents random and short term fluctuations. Therefore, we want to minimize this frequency fluctuation to minimize the phase noise of the oscillator. The oscillator's phase noise is highly dependent on the quality factor of the resonator it uses. By improving the resonator performance, the phase noise will be reduced.

Resonators are not only used in oscillators but also in filters and tuned amplifiers. They naturally oscillate at a given frequency called the resonant frequency and in an ideal resonator, this resonance occurs when the time averaged stored electric and magnetic fields are equal. If the resonator is not ideal, the energy losses will affect the oscillations causing them to attenuate. A measure of this effect and of the sharpness of the resonance is the quality factor or Q factor. High $Q$ values indicate a lower rate of energy loss in the resonator and it can therefore be defined as the ratio of the average energy stored to the energy lost per unit cycle multiplied by $\omega$. In fact, as previously mentioned, one of the most effective ways of minimizing phase noise is by designing a high quality factor resonator [6]. The unloaded quality factor, $Q_{u}$, is the quality factor of the resonator when it is measured by itself whereas the loaded quality factor, $\mathrm{Q}_{\mathrm{L}}$, is the quality factor of the resonator when it is fed by or coupled to an external load. A high loaded quality factor indicates good frequency selectivity and therefore lower phase noise.

\subsection{Thesis Objectives, Contributions and System Concept}

The main objective of this thesis is to determine the feasibility of embedding a precision machined high quality hemispheroidal cavity resonator into a gold plated brass package that houses an E-band oscillator. 
The realization of the overall oscillator system was a collaborative effort with fellow Master of Applied Science student Han-ti Chuang. This study is focused on the design and challenges involved in the high quality factor hemispherical cavity resonator which will be discussed in the following paragraphs. Mention of all oscillator components is given in subsequent Chapters but the design details and challenges particular to this work only are presented in this thesis. The resonator is optimized for use at the $20 \mathrm{GHz}$ sub harmonic and will be used to lower the phase noise of an E-band oscillator also mounted in this package [7]. The $20 \mathrm{GHz}$ frequency of the resonator was chosen as a result of available COTS components for the oscillator's active circuitry.

The resonator, at $20 \mathrm{GHz}$, will be wire bonded to the active circuitry with an internal multiplier bringing the oscillators output frequency up to $40 \mathrm{GHz}$. The amplifier following the signal source allows the power to come up to the level which is sufficient to drive the sub-harmonic mixer which will ultimately be connected to the output in order to raise the frequency up to E-band. Table 1.1 summarizes the required specifications for the VCO and point-to-point E-band link.

Table 1-1: Link and VCO Specifications

\begin{tabular}{|l|l|}
\hline \multicolumn{1}{|c|}{ Parameter } & \multicolumn{1}{c|}{ Required Specification } \\
\hline Frequency & $80 \mathrm{GHz}$ \\
\hline Modulation Type & $256 \mathrm{QAM}$ \\
\hline Data Rate & $1.5 \mathrm{Gbps}$ \\
\hline Oscillator Phase noise & $-106 \mathrm{dBc} / \mathrm{Hz} @ 100 \mathrm{KHz}$ \\
\hline Resonator loaded Q & $>2000$ \\
\hline
\end{tabular}

A schematic of the proposed system concept can be seen in Figure 1-5. A brass package was chosen to minimize the cost of production although the casing and the cavity resonator are both gold plated to improve resonator performance. The millimeter wave substrate chosen was Rogers Duroid 5880 as it presents the lowest loss tangent available; it is soft, easy to work with and is also relatively inexpensive. The active circuitry of the oscillator is located on the top side of the millimeter wave substrate and is 
connected to the resonator, via bond wires, to a microstrip transmission line which couples to an aperture in the ground plane of the millimeter wave substrate.

An analytical and electromagnetic analysis will be performed on the parameters affecting the quality factor of the resonator, such as aperture position and radius in order to optimize the performance and achieve the highest loaded quality factor possible. From a separate analysis [7], the E-band oscillator designed to be mounted on the brass package requires a loaded quality factor of at least 2000 in order to achieve the required phase noise of $-106 \mathrm{dBc} / \mathrm{Hz}$ at $100 \mathrm{KHz}$ offset at $80 \mathrm{GHz}$.

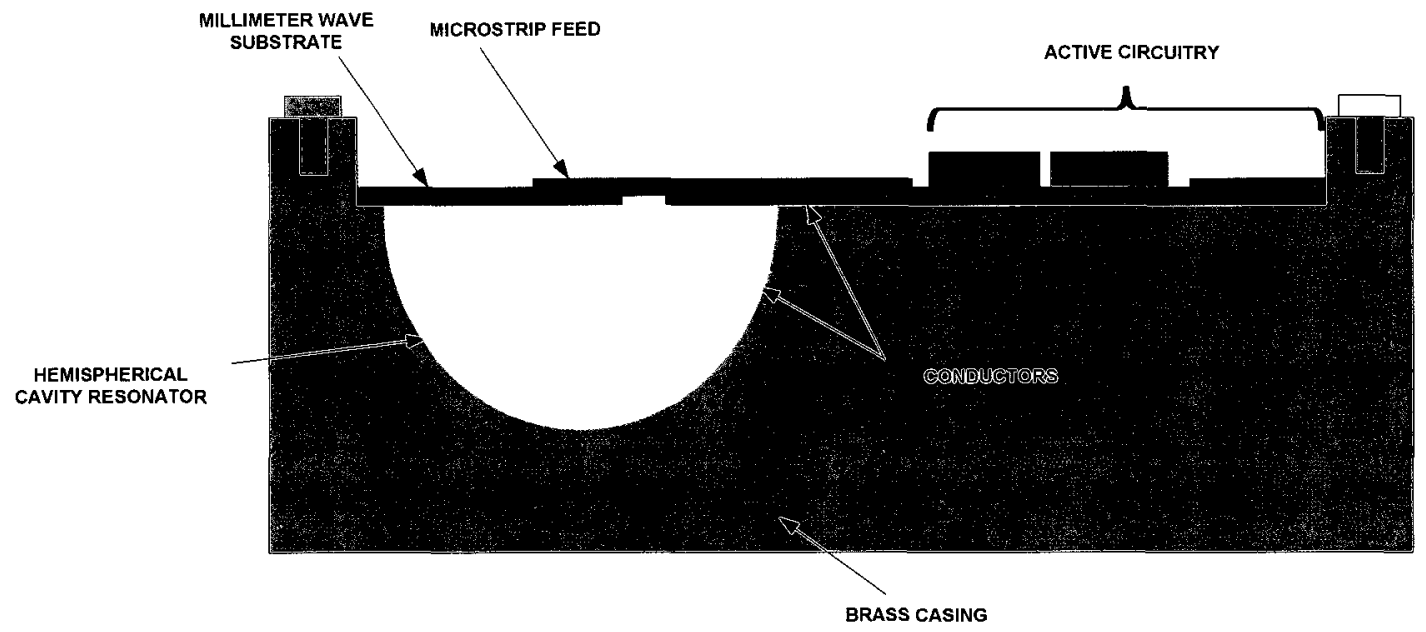

Figure 1-5: Cross section of proposed oscillator package

Also, the resonant frequency and loaded and unloaded quality factors of the resonator will be calculated using equations from hemispherical waveguide cavity theory which were previously studied by Scott R. McLelland's (and upon who's structure this work is based) [8].

Furthermore, it is possible to make the cavity resonator tunable via a simple screw to perturb the electromagnetic fields slightly, thus allowing the frequency to shift. The amount of frequency tuning and associated change in quality factor will be explored. 


\subsection{Thesis Organization}

Further detail and background information on the concepts introduced in Section 1.1 will be described in Chapter 2. This includes an in-depth look at quality factor in relation to phase noise. An overview of basic resonator theory will also be included along with a literature review to compare available types of resonators with relative resonant frequencies and quality factors.

Chapter 3 will present the design of the hemispherical cavity resonator using expressions for resonant frequency, unloaded and loaded quality factors and followed by electromagnetic simulations.

An in-depth sensitivity analysis of the parameters affecting the quality factor of the resonator is presented in Chapter 4, including an analysis of the tuning capability of the resonator. Complete analysis of the oscillator package will also be presented, with a look at the PCB layout issues which might affect the resonator.

Chapter 5 will contain the measured performance of the prototype hemispherical resonator. This includes the comparison with the simulated and calculated performances as stated in Chapter 3.

Finally, Chapter 6 includes a discussion of the results and contributions and discusses the possibilities for future studies in this field. 


\section{Chapter 2}

\section{Microwave Resonators}

\subsection{Basic Background Theory}

The implementation of resonators is fundamental in the design of filters and oscillators. They can however become complicated at microwave frequencies $(300 \mathrm{MHz}$ to $300 \mathrm{GHz}$ ) since transmission lines, waveguides of various shapes or dielectric cavities are used. In addition, the feed and coupling structures to the resonator are very important for resonator performance.

Near the resonant frequency, microwave resonators can be modeled by applying RLC lumped-element equivalent circuits in series or in parallel. The simplest and most ideal resonator consists of two elements, a capacitor and an inductor. In reality though, there are losses, $\mathrm{R}$ and $\mathrm{G}$ elements as shown in Figure 2-1, which are associated with the resonator and are always inevitable in real circuits. 


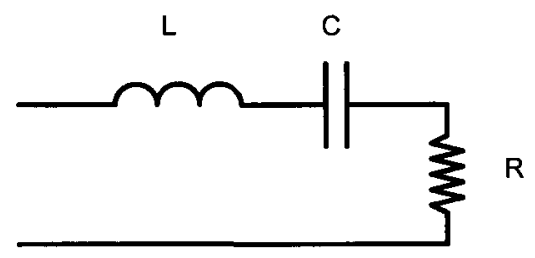

(a)

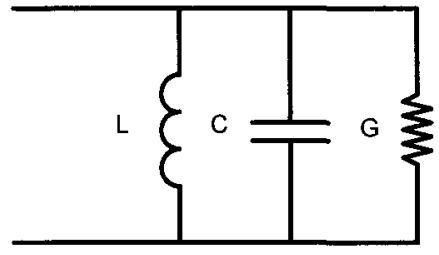

(b)

Figure 2-1: Resonant circuits. (a) Series RLC circuit. (b) Parallel RLC circuit

Some important parameters associated with these circuits are the resonant frequency, $f_{0}$, in Hertz, given by

$$
f_{0}=\frac{1}{2 \pi \sqrt{L C}}
$$

and the quality factor, Q. As mentioned in the previous Chapter, the quality factor of the resonator is used to specify the energy loss and the frequency selectivity of the circuit. It is the average energy stored divided by the energy lost in the system per unit cycle multiplied by $\omega$. The unloaded quality factor, $Q_{u}$, can be calculated from Eqns. (2.1-1) and (2.1-2) for the series and the parallel resonant circuits, respectively.

$$
\begin{aligned}
& Q_{u}=\frac{1}{\omega_{0} R C}=\frac{\omega_{0} L}{R} \\
& Q_{u}=\omega_{0} R C=\frac{R}{\omega_{0} L}
\end{aligned}
$$

Up to this point, the resonator was considered to be on its own, but in order for it to be practical it needs to be coupled to an external load. This is where the external quality factor, $Q_{\mathrm{ext}}$, comes into play as it is the ratio of the energy stored in the resonator to the energy lost in the system outside the connection port. It is however the loaded quality factor, $\mathrm{Q}_{\mathrm{L}}$, which is most important in the design of a resonator as it accounts for both the unloaded and external quality factors. It can be defined as

$$
\frac{1}{Q_{L}}=\frac{1}{Q u}+\frac{1}{Q_{e x t}}
$$


The loaded quality factor will be lower than the unloaded quality factor since it accounts for the effects of the external coupling.

\subsection{Excitation Techniques}

The parameters described in the previous section, such as $f_{0}$ and $\mathrm{Q}_{\mathrm{u}}$, are defined by assuming that the resonator is not connected to an external circuit, in other words, there is no exchange of energy with an external system. In order for the resonator to be practical, it needs to be coupled to the external circuitry. There are various ways of doing so, depending on the type of resonator considered. Some typical coupling techniques are shown in Figure 2-2.

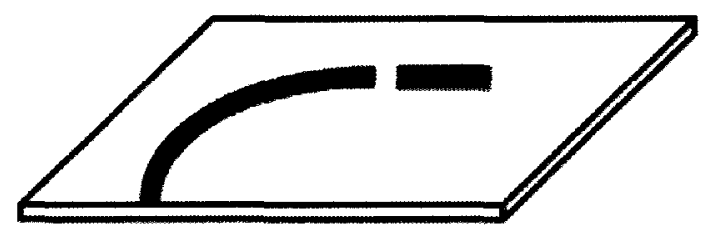

a)

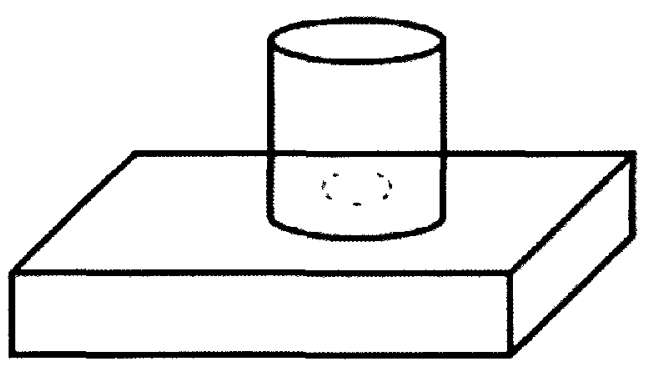

c)

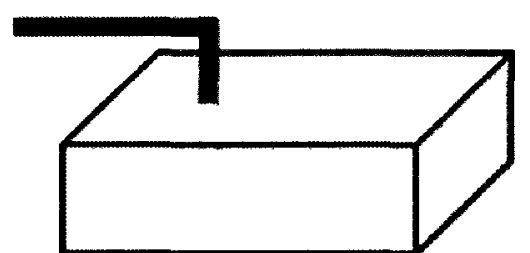

b)

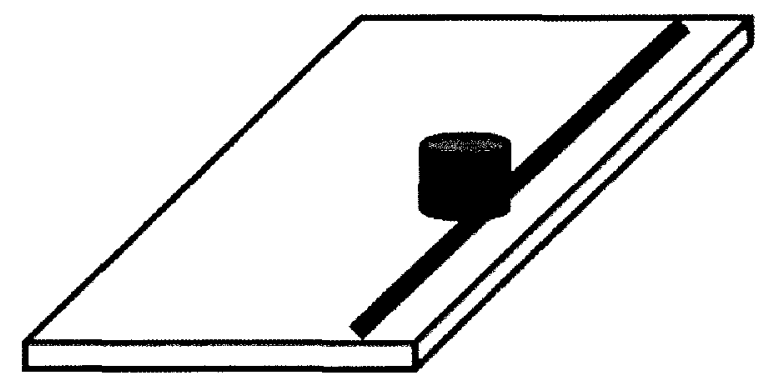

d)

Figure 2-2: Coupling techniques. (a) Microstrip transmission line resonator gap coupled to a microstrip feed line. (b) Rectangular cavity resonator fed by a coaxial probe. (c) Circular cavity resonator aperture coupled to a rectangular waveguide. (d) Dielectric resonator coupled to a microstrip feedline[9]. 
The electromagnetic coupling between a waveguide and a cavity resonator, as shown in (c) of Figure 2-2, will be the technique used in this research. The coupling is usually established via an aperture in a common wall between the cavity and the waveguide. The aperture coupling is arranged in such a way that the excitation of the magnetic field is at its maximum.

The amount of energy coupling to and from the resonator is called the coupling coefficient and is defined as the ratio of the unloaded quality factor to the external quality factor as shown in Eqn. (2.2-0).

$$
k=\frac{Q_{u}}{Q_{e x t}}
$$

If the coupling does not significantly degrade the performance of the resonator and is therefore less than one, the resonator is said to be undercoupled. If the coupling coefficient is equal to one, the resonator is said to be critically coupled as the resonator is losing almost half of the energy it can store to the outside system. With a coupling coefficient greater than one, the resonator is said to be overcoupled and the coupling is severely degrading the performance of the resonator. For oscillator design it is ideal to maximize the energy storage and design an undercoupled resonator. To do so, it must be designed for high loaded quality factor.

The aim of this research is in fact, to design a high loaded quality factor resonator to be applied in a low phase noise oscillator. Phase noise is considered the most critical specification for the design of oscillators. It can be calculated using Leeson's equation [10],

$$
L=10 \log \left\{\frac{G F K T}{2 P}\left[\left(\frac{f_{0}}{2 Q}\right)^{2}\left(\frac{f_{\alpha}}{f^{3}}\right)+\left(\frac{f_{0}}{2 Q}\right)^{2}\left(\frac{1}{f^{2}}\right)+\frac{f_{\alpha}}{f}+1\right]\right\}
$$

where, $f_{\alpha}$ is the active device flicker-corner frequency, $f_{0}$ is the oscillation frequency, and $f$ is the offset frequency. $G$ is the active device gain, $F$ is the noise factor, $K$ is the Boltzman's constant, $T$ is the absolute temperature and $P$ is the power applied to the resonator. The $1 / f$ term is usually ignored due to the dominating factor $1 / f^{2}$. This can be seen in Figure 2-3 in the classical behaviour of oscillator phase noise. For offset 
frequencies higher than half the resonator bandwidth $f_{0} / 2 Q$, the phase noise is mostly determined by the thermal noise of the active device, the noise factor and the power. The region is flat and is called the "noise floor". For offset frequencies between the half bandwidth and the flicker corner frequency, the phase noise follows Leeson's equation and is a combination of loaded quality factor, noise factor and temperature [5]. It increases at a rate of $20 \mathrm{~dB}$ per decade. Finally, in the last region where the flicker noise dominates, the phase noise increases to $30 \mathrm{~dB}$ per decade. The half resonator bandwidth and the flicker frequency are, therefore two of the most important parameters regarding phase noise [10].

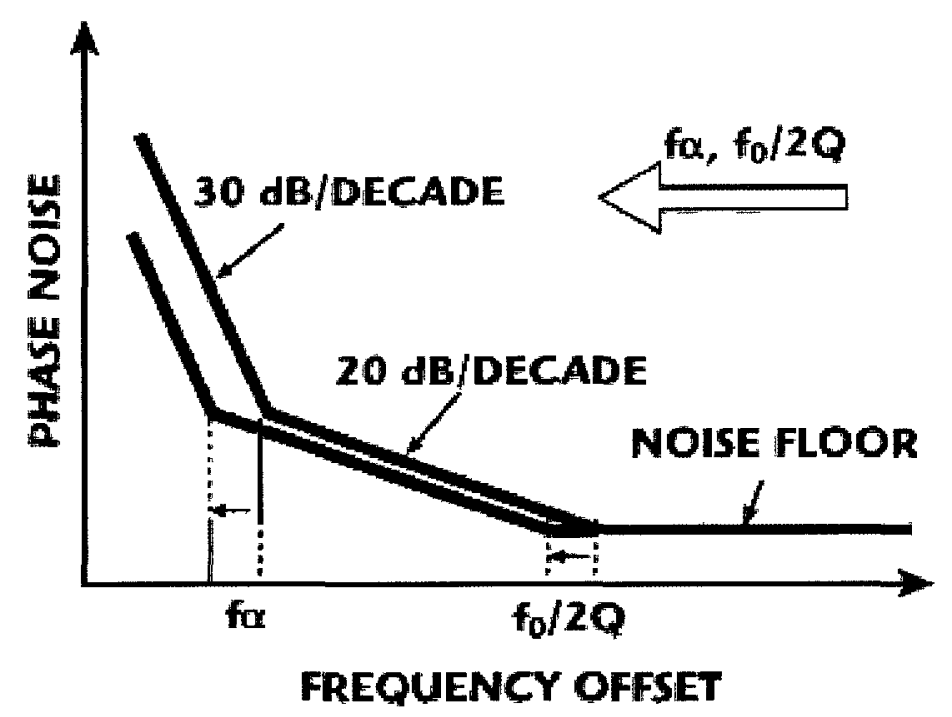

Figure 2-3: Classic oscillator phase noise behavior [10].

\subsection{Literature Review}

Various types of resonators exist on the market, from simple RLC circuits as described in Section 2.1, which yield low quality factors, to more complicated resonators such as dielectric resonators, cavity and quartz resonators that produce some of the highest quality factors ever achieved. Some state of the art micro- and millimetre-wave resonators are shown in Figure 2-4. 
Microwave high-Q resonators are usually made of rectangular or cylindrical waveguides that are expensive, heavy and can be difficult to integrate with monolithic circuits. Their performances however can be quite impressive if no other aspect is an issue.

Quartz crystal resonators are used in many frequency applications due to their stability, small size and low cost. The material properties of single-crystal quartz are stable with time, temperature and other environmental changes. The most attractive feature they possess, is the extremely high quality factor which ranges from 10,000 to $1,000,000$ [11]. The drawback of quartz-crystals is that they are only manufactured for frequencies from a few tens of kilohertz to tens of megahertz and cannot be used for high frequency applications.

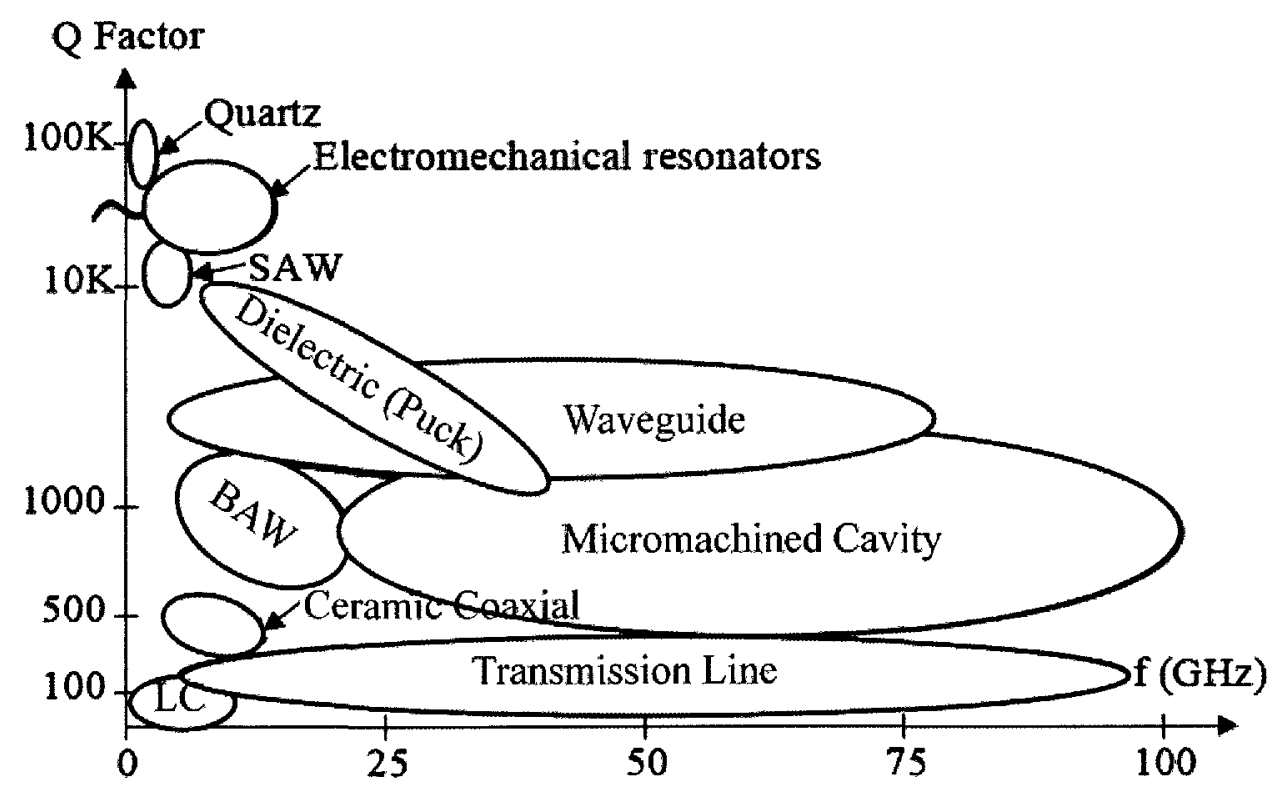

Figure 2-4: State of the art resonators [12].

Surface Acoustic Wave resonators (SAW) and Bulk Acoustic Wave resonators (BAW) are widely used for low-loss filters but are limited to usage up to the Ku-band $(10.95 \mathrm{GHz}-14.5 \mathrm{GHz})$.

Dielectric resonators overcome the limitations of the resonators previously discussed. They are made of low loss, temperature stable, high permittivity and high Q ceramic 
materials, and have a practical frequency range that lies between $2 \mathrm{GHz}$ and $40 \mathrm{GHz}$. In fact, quality factors as high as 10,000 at $4 \mathrm{GHz}$ [13] have been reported using common materials. The major disadvantage of dielectric resonators is their complexity when it comes to the integration on a planar PCB, the difficulty in realizing mass production and the variation of dielectric constant with temperature [14].

Coaxial resonators have various attractive features such as low-loss characteristics and small size but this becomes a limitation for applications above $10 \mathrm{GHz}$ because their miniscule physical dimensions create manufacturing inaccuracies.

Micro-machined cavities seem to be very suitable for millimetre-wave applications in the frequency ranges from $20 \mathrm{GHz}$ to $100 \mathrm{GHz}$ and they also provide fairly high quality factors [15-18].

Waveguide resonators are similar the micro-machined cavities but are difficult to integrate, similar to the dielectric resonators mentioned above. Their size is significantly larger than other resonators available in the microwave region.

Transmission line resonators offer a wide range of frequencies, although they are not feasible at high microwave frequencies and unfortunately do not provide much flexibility in terms of quality factor.

A micro-machined hemispheroidal cavity resonator at W-band, precisely $76.39 \mathrm{GHz}$, reports a measured unloaded quality factor of 1426 and a measured loaded quality factor of 909 has been reported [19]. The cavity is micro-machined using self-limited isotropic etching of a silicon wafer, metalized with gold and soldered to an alumina wafer using a thin layer of indium. The alumina is patterned with a microstrip feed line having an aperture in the ground plane for coupling to the cavity [19]. The shape of the cavity is not perfectly hemispherical, but is considered an oblate hemispheroid. Such a structure, machined in brass, is the main focus of this thesis. 


\section{Chapter 3}

\section{Cavity Resonator Design}

\subsection{The Unloaded Hemispherical Cavity Resonator}

The inspiration behind this research work comes from the encouraging results that the hemispheroidal cavity resonator presents at high frequency. Although micro-machining will be discarded, a different approach will be taken in order to facilitate manufacturing processes.

As mentioned in Section 1.2, the resonator consists of a hemispherical cavity machined in a brass package with a conducting top cover. The brass is coated to a few skin depths with gold to reduce cavity loss. Figure 3-1 shows the spherical coordinate system. Assuming no losses, the boundary conditions of the normal electric field and tangential magnetic field are used to solve Maxwell's equations for the hemispherical resonator structure. 


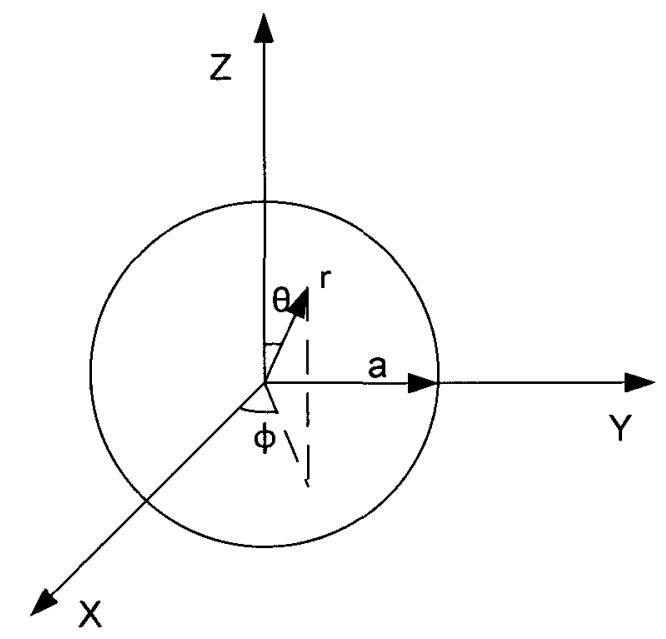

Figure 3-1: Spherical cavity coordinate system [20].

The analysis of the hemispherical cavity resonator begins with the solution of the spherical wave equation (Helmholtz equation) for the components of the electromagnetic fields for the resonant modes. Depending on the mode, the resonant frequencies can be calculated from Eqns. (3.1-0) and (3.1-1) for the TE and TM modes respectively [20].

$$
\begin{aligned}
& \left(f_{r}\right)_{m n p}^{T E}=\frac{u_{n p}}{2 \pi a \sqrt{\epsilon \mu}} \\
& \left(f_{r}\right)_{m n p}^{T M}=\frac{u_{n p}^{\prime}}{2 \pi a \sqrt{\epsilon \mu}}
\end{aligned}
$$

The ordered zeros, $u_{n p}$, of the spherical Bessel function, $\widehat{J_{n}}(u)$, and the ordered zeros, $u_{n p}^{\prime}$, of the derivative of the spherical Bessel function, $\hat{J}_{n}^{\prime}\left(u^{\prime}\right)$, can be found in Table 21 and Table 2-2 respectively. As seen in Eqns. (3.1-0) and (3.1-1), the resonant frequency is independent of $m$ and is directly proportional to $u_{n p}$ and $u_{n p}^{\prime}$. From Tables 2-1 and 2-2 the modes in order of ascending resonant frequencies are $T M_{m, 1,1}, T M_{m, 2,1}, T E_{m, 1,1}$, $T M_{m, 3,1}, T E_{m, 2,1}$, and so on. There are however, some modes that possess the same resonant frequency. These modes are called degenerate modes, for example the three lowest order TM modes, $T M_{0,1,1}, T M_{1,1,1}^{\text {even }}$ and $T M_{1,1,1}^{o d d}$, where the superscripts "even" and 
"odd" indicate the choice of $\cos m \varphi$ or $\sin m \varphi$, respectively, in Eqns. (3.1-2), (3.1-3) and (3.1-4) for the TM mode. The same reasoning applies to the TE modes.

$$
\begin{aligned}
& \left(A_{r}\right)_{0,1,1}=\widehat{J_{1}}\left(2.744 \frac{r}{a}\right) \cos \theta \\
& \left(A_{r}\right)_{0,1,1}^{e v e n}=\widehat{J_{1}}\left(2.744 \frac{r}{a}\right) \sin \theta \cos \theta \\
& \left(A_{r}\right)_{0,1,1}^{\text {odd }}=\widehat{J_{1}}\left(2.744 \frac{r}{a}\right) \sin \theta \sin \theta
\end{aligned}
$$

\begin{tabular}{|c|c|c|c|c|c|}
\hline $\mathrm{p}$ & 1 & 2 & 3 & 4 & 5 \\
\hline 1 & 4.493 & 5.763 & 6.988 & 8.183 & 9.356 \\
\hline 2 & 7.725 & 9.095 & 10.417 & 11.705 & 12.967 \\
\hline 3 & 10.904 & 12.323 & 13.698 & 15.040 & 16.355 \\
\hline 4 & 14.066 & 15.515 & 16.924 & 18.301 & 19.653 \\
\hline 5 & 17.221 & 18.689 & 20.122 & 21.525 & 22.905 \\
\hline
\end{tabular}

Table 3-1: Ordered zeros $u_{n p}$ of $\widehat{J_{n}}(u)$. [20]

Table 3-2: Ordered zeros $u_{n p}^{\prime}$ of ${\widehat{J_{n}}}^{\prime}\left(u^{\prime}\right)$. [20]

\begin{tabular}{|l|l|l|l|l|l|}
\hline $\mathrm{p}$ & & & & & \\
\hline 1 & 1 & 2 & 3 & 4 & 5 \\
\hline 2 & 2.744 & 3.870 & 4.973 & 6.062 & 7.140 \\
\hline 3 & 6.117 & 7.443 & 8.722 & 9.968 & 11.189 \\
\hline 4 & 9.317 & 10.713 & 12.064 & 13.380 & 14.670 \\
\hline 5 & 12.486 & 13.921 & 15.314 & 16.674 & 18.009 \\
\hline
\end{tabular}




\subsection{Resonant Frequency and Cavity Dimensions}

In order to begin the design of the hemispherical resonator, the cavity must be properly sized depending on the operating frequency. The fundamental resonant frequency has been defined by Eqns. (3.1-0) and (3.1-1) for the TE and TM mode, respectively. From the analysis of the unloaded hemispherical cavity resonator in Section 3.1, the fundamental $T M_{011}$ mode results in the smallest cavity size at the desired frequency, therefore rearranging equation 3.1-0 to find the cavity radius results in

$$
a=\frac{u_{11}^{\prime}}{2 \pi f_{o} \sqrt{\epsilon_{o} \mu_{o}}}
$$

where $u_{11}^{\prime}=2.744$, the operating frequency is $f_{o}=20 \mathrm{GHz}$ and $\sqrt{\epsilon \mu}=\sqrt{\epsilon_{o} \mu_{o}}$ since the cavity contains only air. The resulting radius at this frequency is $a=6.54 \mathrm{~mm}$. The cavity is a perfect hemisphere as shown in Figure 3-2.

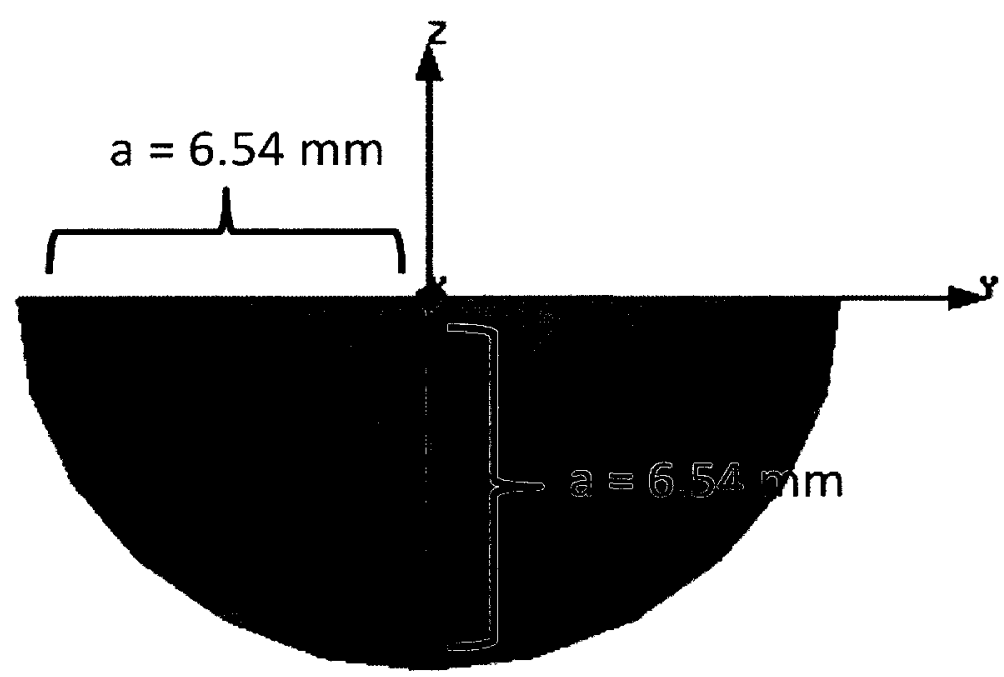

Figure 3-2: Cavity dimensions 


\subsection{The Unloaded Quality Factor}

The mode pattern for one of the dominant modes of the hemispherical cavity can be simulated in Ansoft HFSS using the eigenmode analysis and is shown in Figure 3-3.

High field magnitude

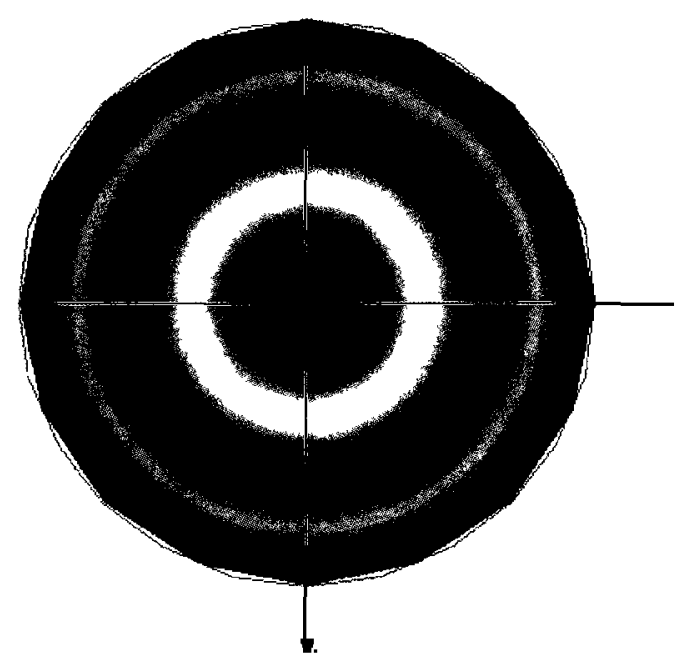

Electric field in xy-plane

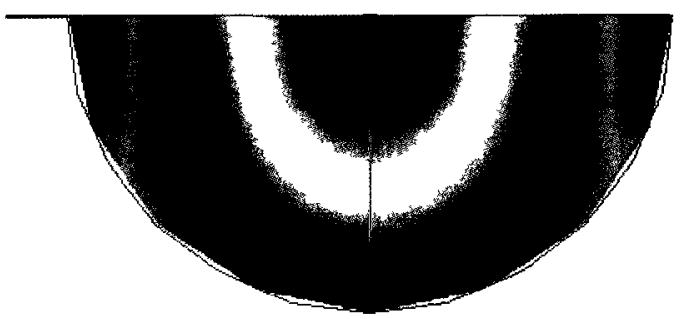

Electric field in zy- \& $x z$-planes

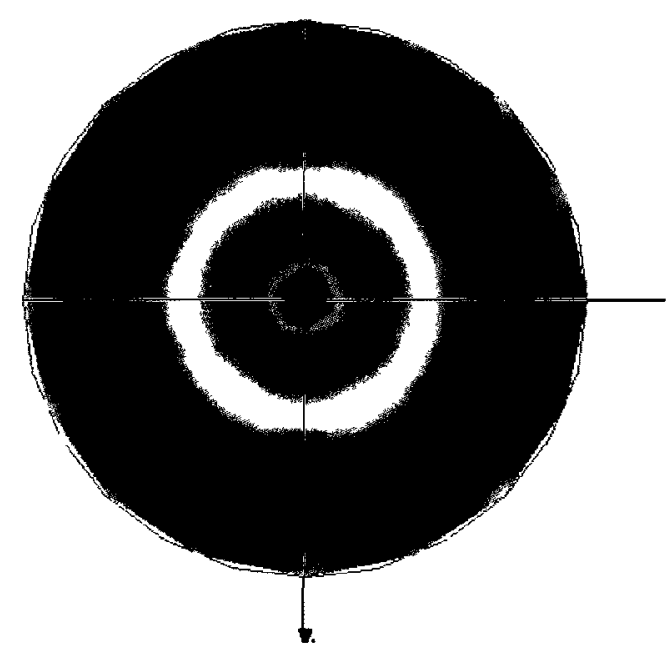

Magnetic field in xy-plane

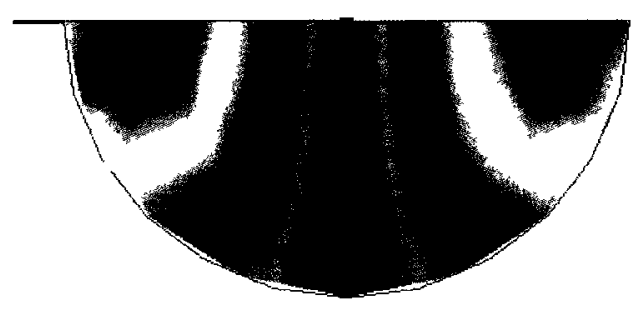

Magnetic field in zy- \& xz-planes

Figure 3-3: Electric and Magnetic fields of the perfect hemispherical cavity at the fundamental $\mathrm{TM}_{011}$ mode.

For TE modes the quality factor due to conductor losses, can be expressed as follows [20]

$$
\left(Q_{c}\right)_{m n p}^{T E}=\frac{\eta u_{n p}}{2 R}
$$


where $R$ is the surface resistance of the conductor. Similarly, for the TM modes the quality can be expressed as [20]

$$
\left(Q_{c}\right)_{m n p}^{T M}=\frac{\eta}{2 R}\left[u_{n p}^{\prime}-\frac{n(n+1)}{u_{n p}^{\prime}}\right]
$$

From Eqn. (3.1-0) it is clear that for the TE modes, as the order, $u_{n p}$, of the mode increases, assuming constant $R$, the quality factor also increases. This indicates that the TE mode has a higher unloaded quality factor than the corresponding TM mode. However, from Eqn. (3.2-0) it is clear that for a given radius $a$, if the order of the mode increases, the resonant frequency will also increase as shown from eigenmode simulations in Table 3-3. Consequently, at the desired resonant frequency, high order modes will provide higher quality factors but will increase the cavity size.

Therefore, in order to maintain optimum quality factor without increasing the size of the resonator, the lowest order TM mode, $\mathrm{TM}_{011}$, is used.

The electric and magnetic field vectors for the fundamental $\mathrm{TM}_{011}$ mode were simulated in HFSS and are shown in Figures 3-4 and 3-5, respectively. As can be seen, the electric field vector is perpendicular to the conductor in the hemisphere, while the magnetic field vector is tangential to the conductor in the hemisphere. 

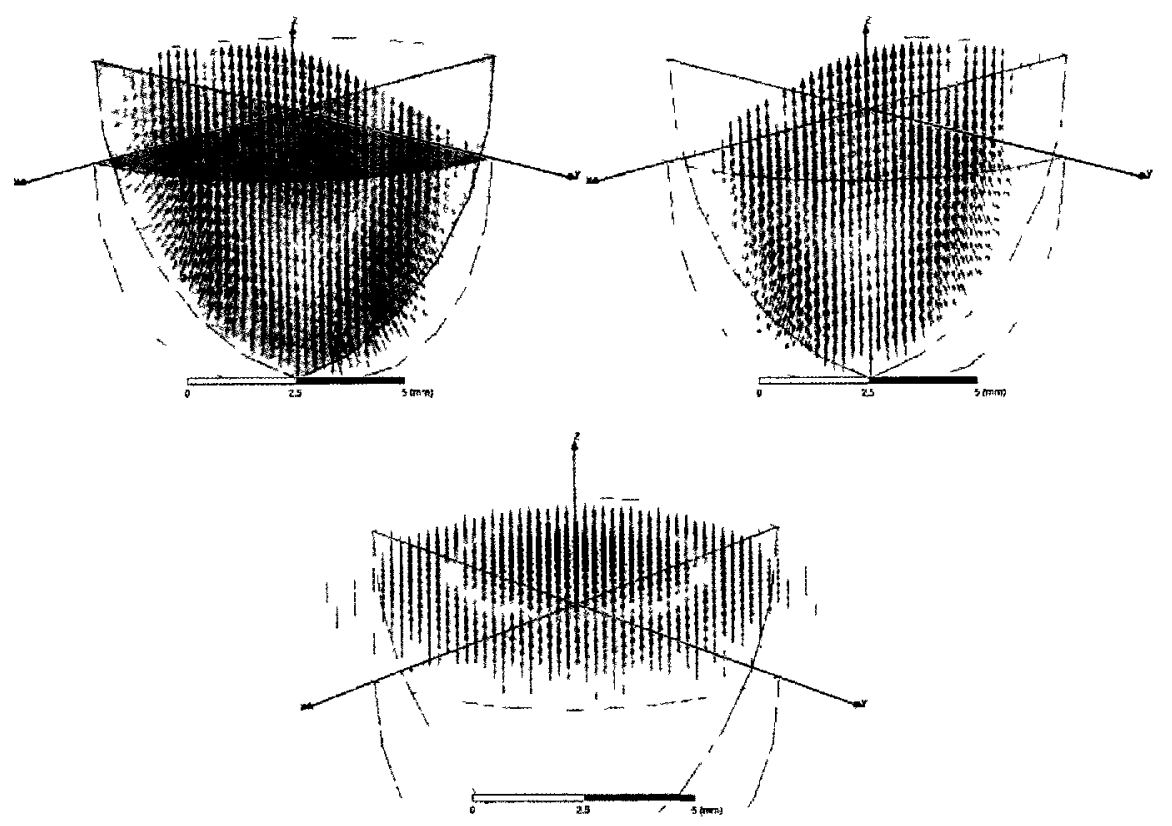

High field magnitude 1 Low field magnitude

Figure 3-4: Electric field mode pattern.
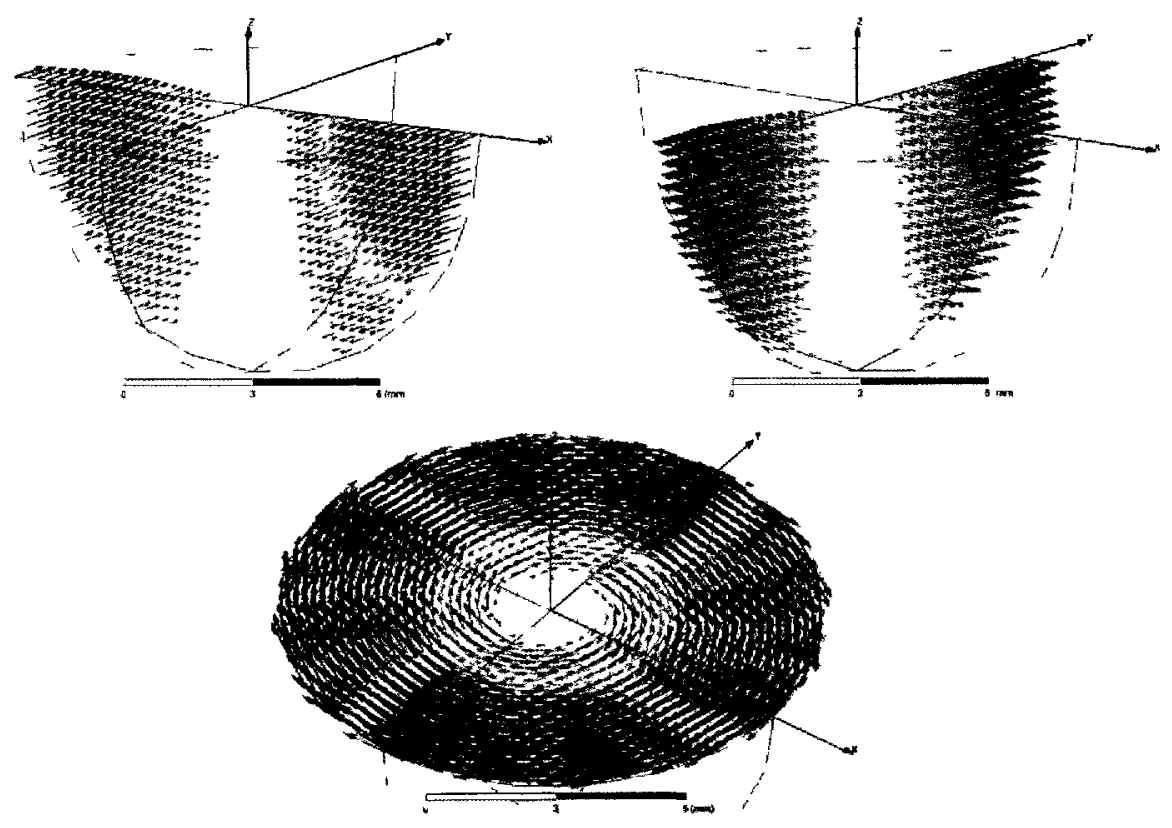

High field magnitude 1 Low field magnitude

Figure 3-5: Magnetic field mode pattern. 
The field components of the $\mathrm{TM}_{011}$ mode of the perfect hemispherical cavity are [8]:

$$
\begin{aligned}
& E_{r}=\frac{2 \cos \theta}{u_{11}^{\prime} r^{3}}\left[a \sin \left(u_{11}^{\prime} \frac{r}{a}\right)-u_{11}^{\prime} r \cos \left(u_{11}^{\prime} \frac{r}{a}\right)\right] \\
& E_{\theta}=\frac{\sin \theta}{u_{11}^{\prime} a r^{3}}\left[a^{2} \sin \left(u_{11}^{\prime} \frac{r}{a}\right)-u_{11}^{\prime} \operatorname{arcos}\left(u_{11}^{\prime} \frac{r}{a}\right)-u_{11}^{\prime 2} r^{2} \sin \left(u_{11}^{\prime} \frac{r}{a}\right)\right] \\
& E_{\phi}=0 \\
& H_{r}=0 \\
& H_{\theta}=0 \\
& H_{\phi}=\frac{\sin \theta}{u_{11}^{\prime} r^{2}}\left[a \sin \left(u_{11}^{\prime} \frac{r}{a}\right)-u_{11}^{\prime} r \cos \left(u_{11}^{\prime} \frac{r}{a}\right)\right]
\end{aligned}
$$

The unloaded quality factor for the fundamental $\mathrm{TM}_{011}$ mode of the hemispherical cavity resonator is [20]

$$
Q=0.573 \frac{\eta}{R}
$$

where $\eta$ is the intrinsic impedance of free space and is equal to $377 \Omega$. $\mathrm{R}$ is the surface resistance and can be calculated by [20]

$$
R=\sqrt{\frac{2 \pi f \mu_{0}}{2 \sigma}}
$$

where $\sigma$ is the conductivity of the metal. The brass will be gold-plated to a thickness of several skin depths. In good conductors, the skin depth varies approximately as the inverse square root of the conductivity. The effect is called the "skin effect" and it causes the effective resistance of the conductor to increase at high frequencies. It is an important factor to keep into consideration as it depends highly on the frequency, in fact the higher the frequency the smaller the skin depth. A well-known formula for skin depth or in other words, the characteristic depth of penetration, is presented in Eqn. (3.3-10).

$$
\delta=\sqrt{\frac{2 \rho}{2 \pi f \mu_{o} \mu_{R}}}
$$


Where $\delta_{s}$ is the skin depth, $\rho$ is the bulk resistivity in ohm $/ \mathrm{m}, f$ is the frequency in $\mathrm{Hz}, \mu_{0}$ is the permeability of free space and $\mu_{R}$ is the relative permeability. A graph of frequency versus skin depth for various materials can be found in Figure 3-6. The skin depth for most conductors is roughly $0.5-0.6 \mu m$ at $20 \mathrm{GHz}$.

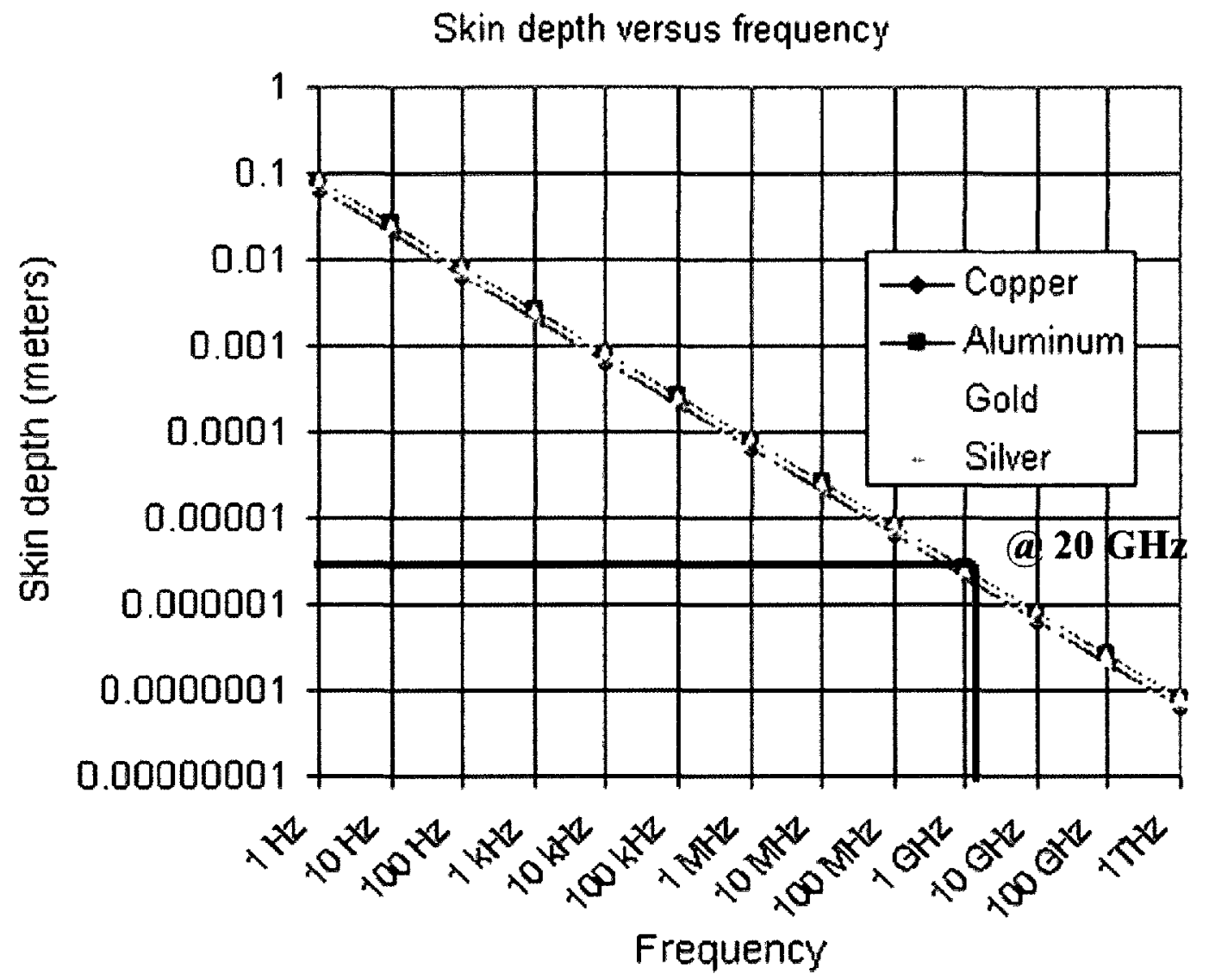

Figure 3-6: Skin depth versus Frequency for different materials [21]

From Table 3-3 of the eigenmode simulations, for a resonant frequency of $20 \mathrm{GHz}$, and a conductivity of gold $\sigma=4.52 \times 10^{7} \mathrm{~S} \cdot \mathrm{m}^{-1}$, the unloaded quality factor, $\mathrm{Q}_{\mathrm{u}}$, is 5180 . This calculated value is only valid for the fundamental $\mathrm{TM}_{011}$ mode and higher order modes would require different equations and are not presented. Simulated unloaded quality factors are however presented. 
Table 3-3: Eigenmode simulations featuring various modes.

\begin{tabular}{|c|c|c|c|c|}
\hline Mode & $\begin{array}{c}\text { Calculated } \\
\text { Frequency } \\
\mathbf{( G H z )}\end{array}$ & $\begin{array}{c}\text { Simulated } \\
\text { Frequency } \\
\mathbf{( G H z )}\end{array}$ & $\begin{array}{c}\text { Calculated } \\
\text { Unloaded Q }\end{array}$ & $\begin{array}{c}\text { Simulated } \\
\text { Unloaded Q }\end{array}$ \\
\hline $\mathrm{TM}_{011}$ & 20.03 & 20.27 & 5180 & 4860 \\
\hline $\mathrm{TM}_{\mathrm{m} 21}$ & 28.25 & 28.55 & - & 5559 \\
\hline $\mathrm{TE}_{\mathrm{m} 11}$ & 32.80 & 28.55 & - & 5543 \\
\hline $\mathrm{TM}_{\mathrm{m} 31}$ & 36.30 & 33.12 & - & 6550 \\
\hline
\end{tabular}

\subsection{The Loaded Quality Factor}

In order for the resonator to be useful, it must be coupled to an external circuit by some kind of excitation technique as mentioned in Section 2.2. The addition of any of these excitation techniques will however, modify the overall performance and quality factor of the resonator as it presents losses and characteristics of its own. The new quality factor to take into account is called the loaded quality factor, $Q_{L}$, and will be lower than the unloaded quality factor due to the losses from the coupling. As the coupling of the resonator becomes weaker, the loaded quality factor increases and consequently, the phase noise will decrease, improving the overall performance of the oscillator.

Although there are a wide range of coupling techniques, aperture coupling theory will be used. Most of the aperture coupling theory is attributed to H. A. Bethe [22] as he was one of the first authors to develop the theory, although it was later adopted and modified by Marcuvitz [23] and Collin [24]. Another important author who extended Bethe's theory was $\mathrm{H}$. Wheeler [25]. Wheeler analyzed aperture coupling between a cavity and transmission line by considering two symmetrical coupling problems: 
- The coupling between two identical transmission lines through a small aperture in a common wall, characterized by a normalized reactance $x$.

- The coupling between two identical cavities through the same aperture, characterized by a coupling factor $k$.

Assuming that the unloaded quality factor of the cavity is known, the loaded quality factor can be expressed as

$$
\begin{aligned}
& \frac{1}{Q_{L}}=\frac{1}{Q_{u}}+\frac{1}{Q_{e x t}} \\
& \frac{1}{Q_{L}}=\frac{1}{Q_{u}}+k x
\end{aligned}
$$

where $x$ is the normalized reactance of the coupling aperture. This theory is valid only if the aperture size is small compared to the wavelength of the electromagnetic field, and it is far away from any perturbations in the wall and if the wall is a perfect conductor.

The cavity to cavity coupling coefficient can be expressed in terms of "cavity effective volume", $V_{c a v}$, and "aperture effective volume", $V_{a p}$. The cavity effective volume is defined as the volume that, when uniformly filled with the field existing at the aperture, stores and amount of energy equivalent to that stored by the actual cavity. The aperture effective volume is related to the polarizability of the aperture. Therefore, the coupling coefficient in terms of effective volume is $[8,25]$

$$
k=\frac{1}{4} \frac{V_{a p}}{V_{c a v}}
$$

The aperture effective volume depends on aperture size, shape and orientation. Although there are many aperture shapes which could also provide excitation to the cavity, as shown in Figure 3-7, a circular aperture was chosen. Using a non-circular aperture does not provide a significant advantage in this case since the goal is not to optimize the coupling coefficient. The aperture shape is chosen to optimize the loaded 
quality factor. Any aperture shape that gives an external quality factor of at least two orders of magnitude higher than the unloaded quality factor is sufficient to achieve a good enough loaded quality factor. It is also well known that the orientation of noncircular apertures will affect the coupling [26, 27], for example non-centered apertures on a wall, but this problem can be ignored since we are using a circular aperture which is independent of orientation. Once modes are excited in the sphere, the location of the aperture also becomes relevant as the desired mode $\left(T M_{011}\right)$ must be excited. To guarantee mostly magnetic coupling, the aperture must be positioned at maximum magnetic field.
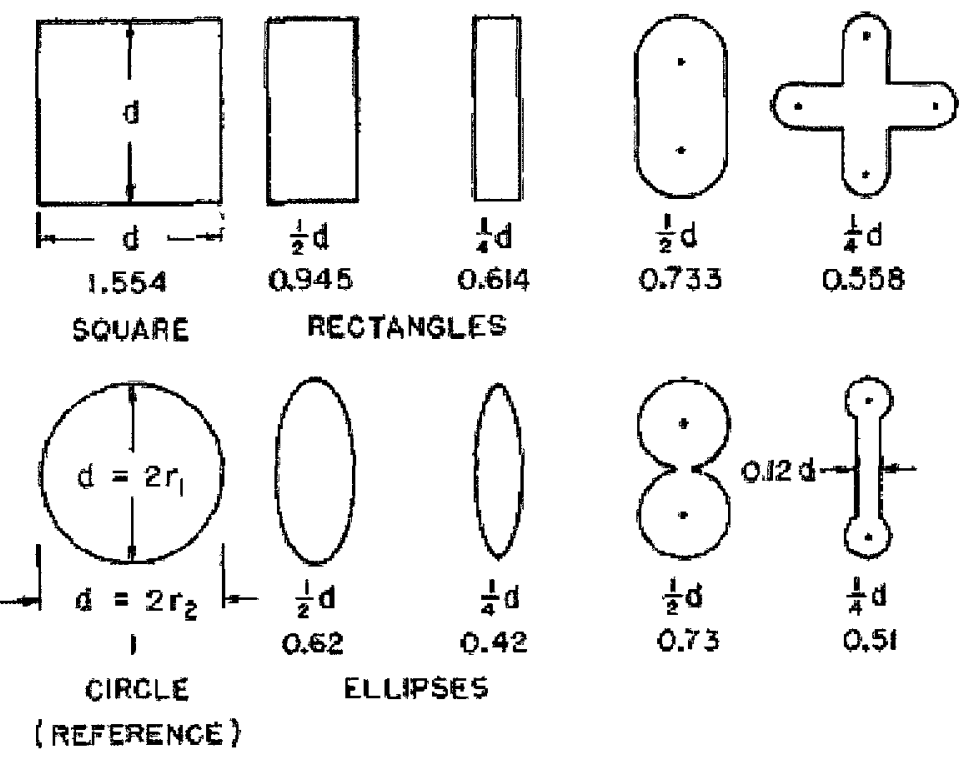

Figure 3-7: Various shapes of coupling apertures [25]

Using the theoretical and measured polarizabilities, Wheeler calculated the "effective volume" of a circular aperture with radius $r_{a}$. For magnetic coupling this is [25]

$$
V_{a p}=\frac{16}{3} r_{a}^{3}
$$

The cavity effective volume then satisfies the following relation $[28,29]$ 


$$
\frac{1}{2} \mu\left|\overrightarrow{H_{a p}}\right|^{2} V_{c a v}=\frac{1}{2} \mu \int_{c a v}\left|\overrightarrow{H_{\emptyset}}\right|^{2} d V
$$

where $\overrightarrow{H_{a p}}$ is the tangential magnetic field at the location of the aperture but without the aperture present. By simplifying Eqn. (3.4-4) and substituting Eqn.(3.3-7), Eqn. (3.4-4) becomes [8]

$$
\begin{gathered}
\int_{c a v}\left|H_{\emptyset}\right|^{2} d V=\int_{r=0}^{a} \int_{\theta=0}^{\pi} \int_{\emptyset=0}^{2 \pi}\left(\frac{\sin \theta}{r^{2} u_{11}^{\prime}}\left[a \sin \left(u_{11}^{\prime} \frac{r}{a}\right)-u_{11}^{\prime} r \cos \left(u_{11}^{\prime} \frac{r}{a}\right)\right]\right)^{2} r^{2} \sin \theta d \emptyset d \theta d r \\
=\frac{2 \pi a}{3 u_{11}^{\prime 2}}\left(u_{11}^{\prime 2}+u_{11}^{\prime} \cos u_{11}^{\prime} \sin u_{11}^{\prime}+2 \cos ^{2} u_{11}^{\prime}-2\right)
\end{gathered}
$$

As mentioned previously, the location of the aperture is an important parameter and also depends on the magnetic field. In fact, the aperture is positioned where the magnetic field is highest. This choice allows the strongest coupling for the smallest possible aperture. The location of the aperture is only a function of the vector $r$ as shown in Figure 3-1 and $\theta=\frac{\pi}{2}$ since the aperture is located on the $x y$ plane. The radial location for maximum magnetic field, $r_{\max }$, is determined by solving $\frac{d}{d r} \overrightarrow{H_{\varnothing}}\left(r_{\max }, \frac{\pi}{2}\right)=0$ for $r_{\max }$. Differentiating Eqn. (3.3-7) with $\theta=\frac{\pi}{2}$ results in [8]

$$
\frac{d}{d r} \overrightarrow{H_{\varnothing}}\left(r, \theta=\frac{\pi}{2}\right)=\left(\frac{r^{2} u_{11}^{\prime 2}-2 a^{2}}{a r^{3} u_{11}^{\prime}}\right) \sin \left(u_{11}^{\prime} \frac{r}{a}\right)+\frac{2}{r^{2}} \cos \left(u_{11}^{\prime} \frac{r}{a}\right)
$$

Setting Eqn. (3.4-6) equal to zero and solving for $r$ gives the location of maximum magnetic field and therefore the location of the aperture at $r_{\max }=5 \mathrm{~mm}$.

Without the aperture present, the tangential magnetic field at the centre of the aperture location is $\overrightarrow{H_{\emptyset}}\left(r=r_{\text {max }}, \theta=\frac{\pi}{2}\right)$ and the field can be assumed uniform over the aperture with the magnitude equal to the one at the centre. That is [8] 


$$
\left|\overrightarrow{H_{a p}}\right|=\frac{a \sin \left(u_{11}^{\prime} \frac{r_{\max }}{a}\right)-u_{11}^{\prime} r_{\max } \cos \left(u_{11}^{\prime} \frac{r_{\max }}{a}\right)}{u_{11}^{\prime} r_{\max }^{2}}
$$

At this point, the "cavity effective volume" can be calculated by substituting Eqns. (3.45) and (3.4-7) into Eqn. (3.4-4):

$$
V_{c a v}=\frac{2 \pi a r_{\max }^{4}\left(u_{11}^{\prime 2}+u_{11}^{\prime} \cos u_{11}^{\prime} \sin u_{11}^{\prime}+2 \cos ^{2} u_{11}^{\prime}-2\right)}{3\left[a \sin \left(u_{11}^{\prime} \frac{r_{\max }}{a}\right)-u_{11}^{\prime} r_{\max } \cos \left(u_{11}^{\prime} \frac{r_{\max }}{a}\right)\right]^{2}}
$$

Finally, by substituting Eqns. (3.4-3) and (3.4-8) into Eqn. (3.4-2), the cavity to cavity coupling coefficient, $k$, can be found.

As mentioned before and as shown in Eqn. (3.4-1), the normalized reactance must also be calculated. The coupling between two identical microstrips of height $h$, line width $W$ and substrate $\varepsilon_{r}$, as shown in Figure 3-8 can be modelled by considering Figure 3-9.
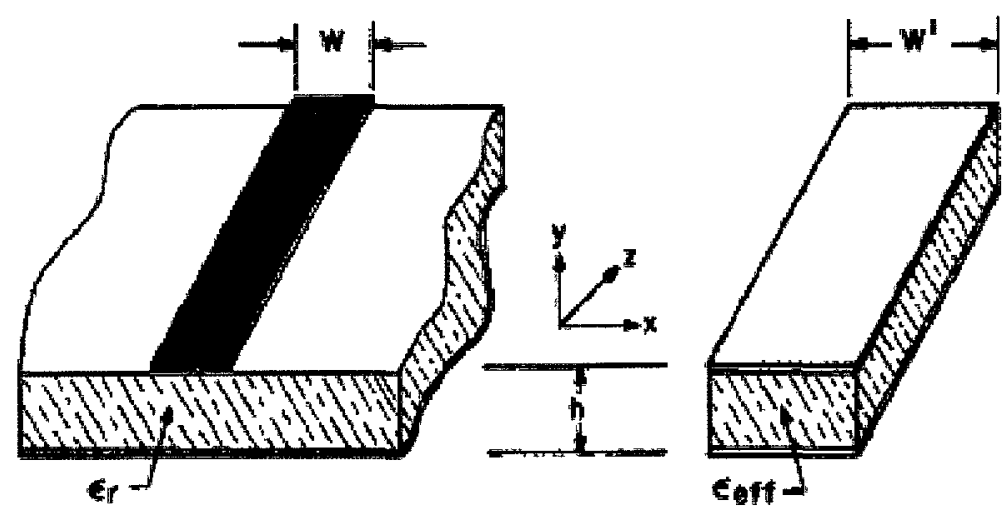

Figure 3-8: Parallel Plate waveguide cross section of a microstrip transmission line [28] 


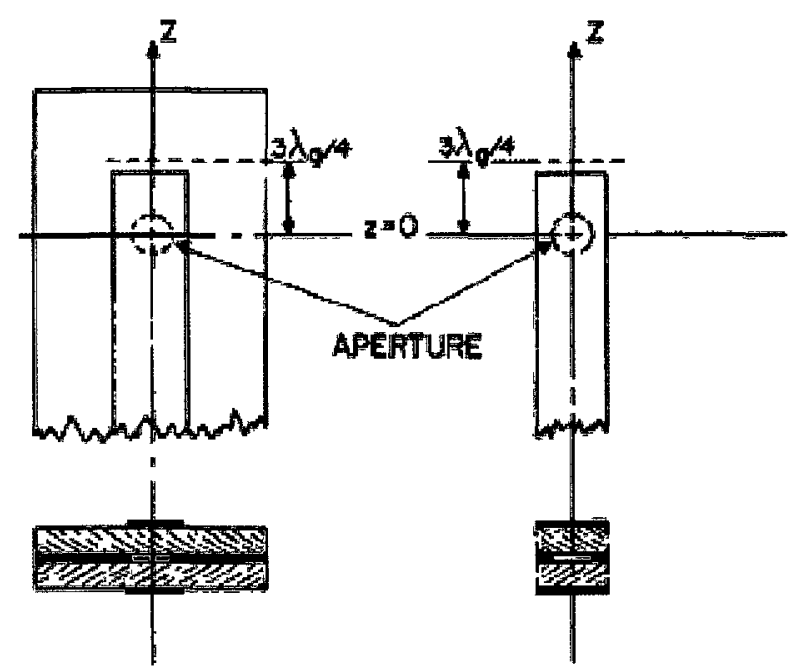

Figure 3-9: Aperture coupling between two identical microstrips [28]

The waveguides consist of two microstrip lines with an aperture of diameter $d$, located in the common ground plane. The microstrips are terminated at one end by an open circuited stub an odd number of quarter wavelengths past the aperture serving to transform the open circuit into a short circuit (maximum magnetic field) at the location of the aperture, allowing for the coupling to be mostly magnetic [29].

Using the microstrip parameters $\left(Z_{o}, \omega_{\mu s t r i p}, h_{\mu s t r p}\right.$ and $\left.\varepsilon_{r}\right)$ that will be described in Section 3.5 , the effective width of the microstrip line is given by

$$
W_{e f f}=\omega_{\mu s t r i p}+\left(\frac{h_{\mu s t r i p}}{z_{o}} \frac{377}{\sqrt{\epsilon_{e f f, d c}}}-\omega_{\mu s t r i p}\right)\left(1+\frac{2 \omega_{\mu s t r i p} f \sqrt{\varepsilon_{r}}}{3 \times 10^{8}}\right)^{-1}
$$

where $\epsilon_{e f f, d c}$ for quasi-static conditions is taken to be 6.674 . The normalized reactance is [8]

$$
x=\left|\frac{T}{2 j(1-T)}\right|
$$

where the transmission coefficient, $T$, is

$$
T=\frac{2 \pi j}{s \lambda_{g}} \cdot M \cdot 2 H_{o}^{2}
$$


where $\mathrm{M}$ is the magnetic polarizability, $M=\frac{4}{3} r_{a}^{3}$, and $S$ is a normalizing factor. Equation 3.4-11 assumes that due to the open circuit stub, no electric field is present at the aperture and there is a uniform magnetic field component over the aperture perpendicular to the z-axis with magnitude $2 H_{o}^{2}$. Substituting Eqn. (3.4-11) into Eqn. (3.4-10), the normalized reactance can be calculated as a function of aperture radius, $r_{a}$

$$
x=\left|\frac{4 \pi r_{a}^{3} \sqrt{\epsilon_{e f f}}}{3 \lambda_{o} W_{e f f} h-j 8 \pi r_{a}^{3} \sqrt{\epsilon_{e f f}}}\right|
$$

Finally, the loaded quality factor $\mathrm{Q}_{\mathrm{L}}$ from Eqn. (3.4-1) can be calculated since all the parameters are known except for the aperture radius. Figure 3-10 shows the calculated quality factor as a function of aperture radius.

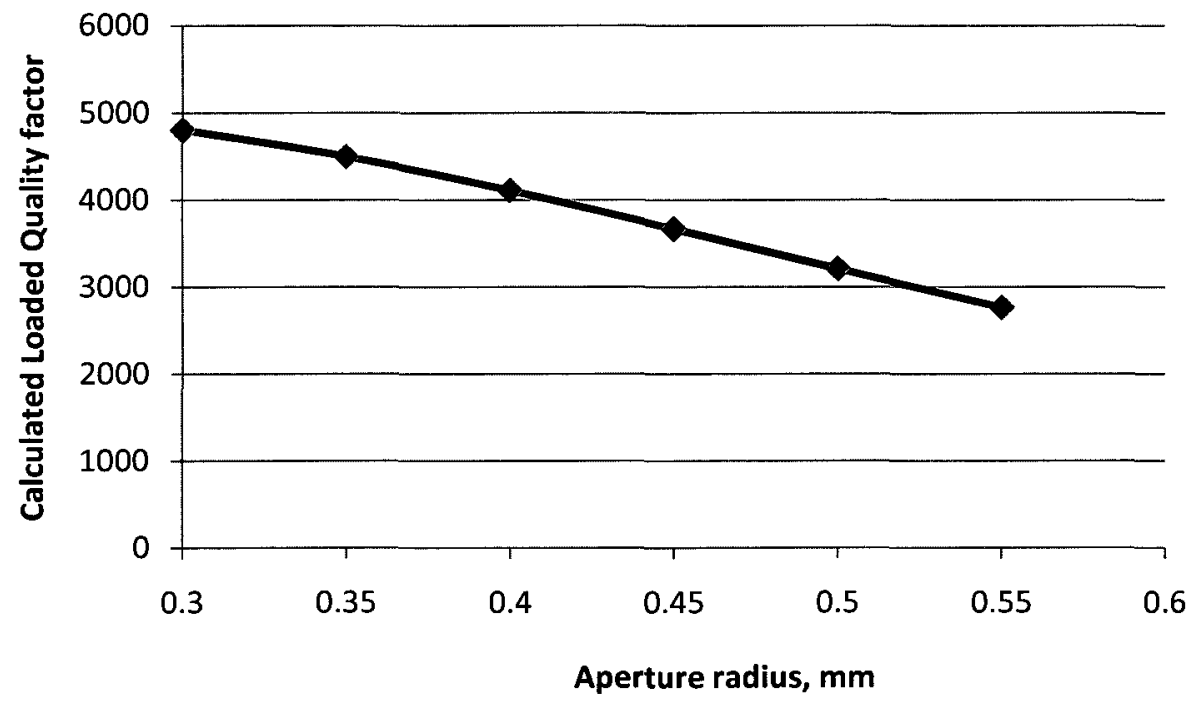

Figure 3-10: Calculated loaded quality factor as a function of aperture radius 


\subsection{Resonator Model}

The hemispherical cavity resonator described in the previous sections has been modelled in Ansoft HFSS and is shown in Figure 3-11.

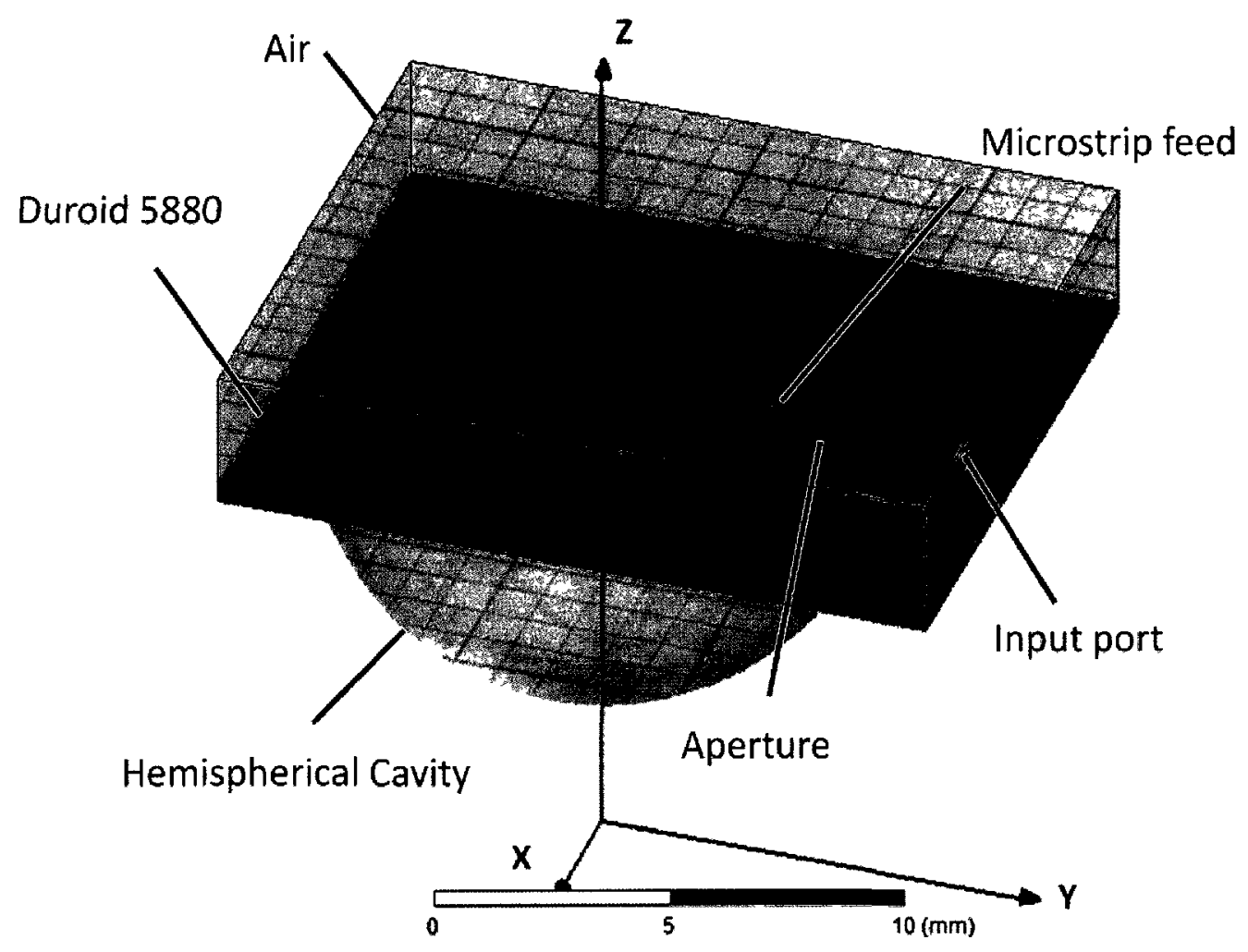

Figure 3-11: 3D view of resonator model.

The cavity resonator is coupled to a microstrip transmission line through a clearance in the ground plane.

Rogers Duroid 5880 [30] was chosen as the millimeter wave material due to its extremely low loss tangent and availability. Specifically, 10 mil thick $\left(h_{\mu s t r p}=254 \mu \mathrm{m}\right)$ Duroid 5880, with a loss tangent, $\tan \delta=0.0009$, a dielectric constant, $\varepsilon_{\mathrm{r}}=2.2$ and $1 / 2$ ounce of copper per $f^{2}\left(\sigma=5.8 \times 10^{7} \mathrm{~S} / \mathrm{m}\right)$. Table 3-4 summarized the properties and presents some comparisons with other common millimeter wave materials. Although 
alumina has a lower loss tangent than the Duroid, it can become very expensive and along with LTCC, has a slower turn-around time. A great advantage of Rogers Duroid 5880 is that is can be easily cut, sheared and machined into various shapes. It is resistant to all solvents and reagents, whether hot or cold, which are usually used in etching printed circuits or in plating edges and holes.

Table 3-4: Comparison of millimeter wave materials.

\begin{tabular}{|c|c|c|c|c|}
\hline Material & $\begin{array}{c}\text { Relative permittivity, } \\
\varepsilon_{\mathbf{r}}\end{array}$ & $\begin{array}{c}\text { Loss Tangent, } \\
\tan \delta\end{array}$ & Cost, $\mathbf{S}$ & $\begin{array}{c}\text { Fabrication } \\
\text { time }\end{array}$ \\
\hline Duroid 5880 & 2.2 & 0.0009 & $\sim 160(5 \mathrm{~cm} \times 5 \mathrm{~cm}$ tile $)$ & $3-5$ days \\
\hline Alumina & 9.9 & 0.0001 & $\sim 5,000(5 \mathrm{~cm} \times 5 \mathrm{~cm}$ tile $)$ & 5 weeks \\
\hline LTCC & 7.1 & 0.001 & $\sim 18,000(30 \mathrm{~cm} \times 30 \mathrm{~cm})$ & 3 weeks \\
\hline
\end{tabular}

The parameters for the feed substrate were calculated using Agilent LineCalc software from ADS. A $Z_{o}=50 \Omega, 17 \mu m$ thick gold microstrip line has a width $w_{\mu s t r i p}=763.62 \mu \mathrm{m}$ at $20 \mathrm{GHz}$. The microstrip line also includes an open circuit $\lambda / 4$ stub of length $2.77 \mathrm{~mm}$ past the aperture, which serves to transform the open circuit at the end, to a short circuit at the centre of the aperture, as described in Section 3.4. In addition, the hemispherical cavity resonator modeled in HFSS has nominal parameters of radius $a=6.54 \mathrm{~mm}$, gold walls (with conductivity, $\sigma=4.1 \times 10^{7} \mathrm{~S} / \mathrm{m}$ ) and is filled with air.

An eigenmode simulation with various materials has been performed on the hemispherical cavity in order to determine which material presents the highest quality factor while still keeping in mind issues such as cost and plating time. Table 3-5 shows eigenmode unloaded quality factor values for some of the most common materials which can be used for plating on the hemispherical cavity resonator. 
Table 3-5: Eigenmode unloaded Q factors for different JIG plating materials.

\begin{tabular}{|c|c|c|}
\hline Material & Conductivity, S/m & Unloaded Quality factor \\
\hline Silver & $6.1 \times 10^{7}$ & 5923 \\
\hline Copper & $5.8 \times 10^{7}$ & 5775 \\
\hline Gold & $4.1 \times 10^{7}$ & 4856 \\
\hline Aluminum & $3.8 \times 10^{7}$ & 4675 \\
\hline Tungsten & $1.8 \times 10^{7}$ & 3235 \\
\hline Brass & $1.5 \times 10^{7}$ & 2937 \\
\hline
\end{tabular}

Gold was the material chosen in this project, and although silver presented the highest unloaded quality factor, one of its major disadvantages is that it oxidizes very quickly and is also very expensive. Copper on the other hand, is much more inexpensive than some of the other materials such as silver and gold but is highly unavailable for plating. There is a contamination issue associated with copper as it does not adhere properly to the brass. Laboratories use it solely if copper is the only material being used in the entire jig fabrication facility. Brass presented the lowest unloaded quality factor, confirming that the jig should be plated in order to minimize the losses due to its conductivity.

\subsection{Loaded Simulations}

The hemispherical cavity resonator geometry shown in Figure 3-11 was simulated using DrivenModal analysis in Ansoft HFSS by a 2D port solution at the input of the microstrip line at a frequency of $20 \mathrm{GHz}$.

Figure 3-12 shows the simulated $S_{l 1}$ as a function of frequency, for aperture radii, (r_cylinder), varying from $0.3 \mathrm{~mm}$ to $0.6 \mathrm{~mm}$. It can be concluded that for increasing aperture radii, the frequency increases slightly with a smaller degree of coupling. 


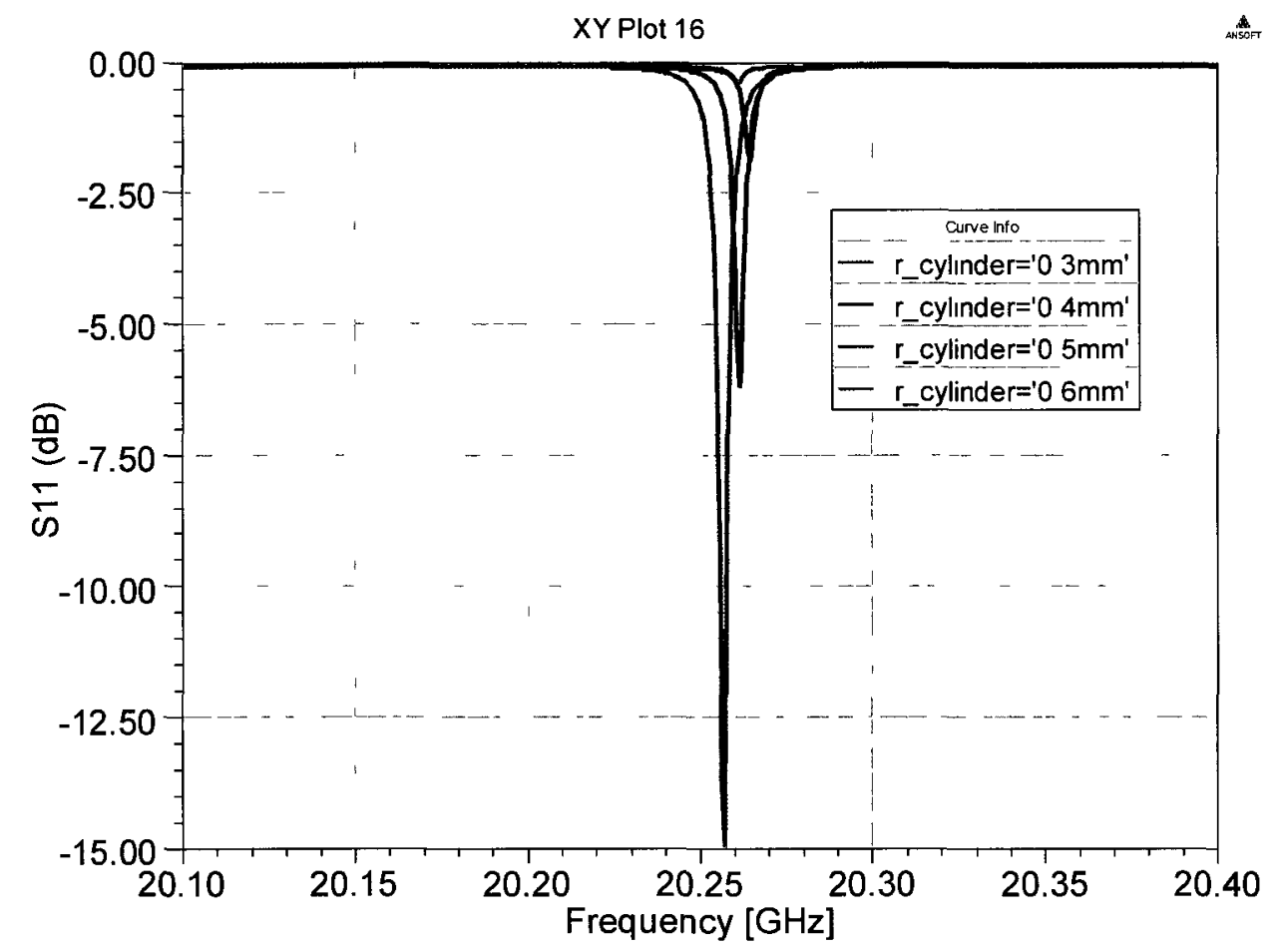

Figure 3-12: Simulated $S_{11}$ of hemispherical cavity resonator for various aperture radii.

Figure 3-13 proves that the cavity resonator is effectively undercoupled as none of the resonant circles on the Smith Chart enclose the $50 \Omega$ point. As the aperture increases, the circle on the Smith chart becomes larger, confirming that the smaller the aperture, the weaker the coupling. 


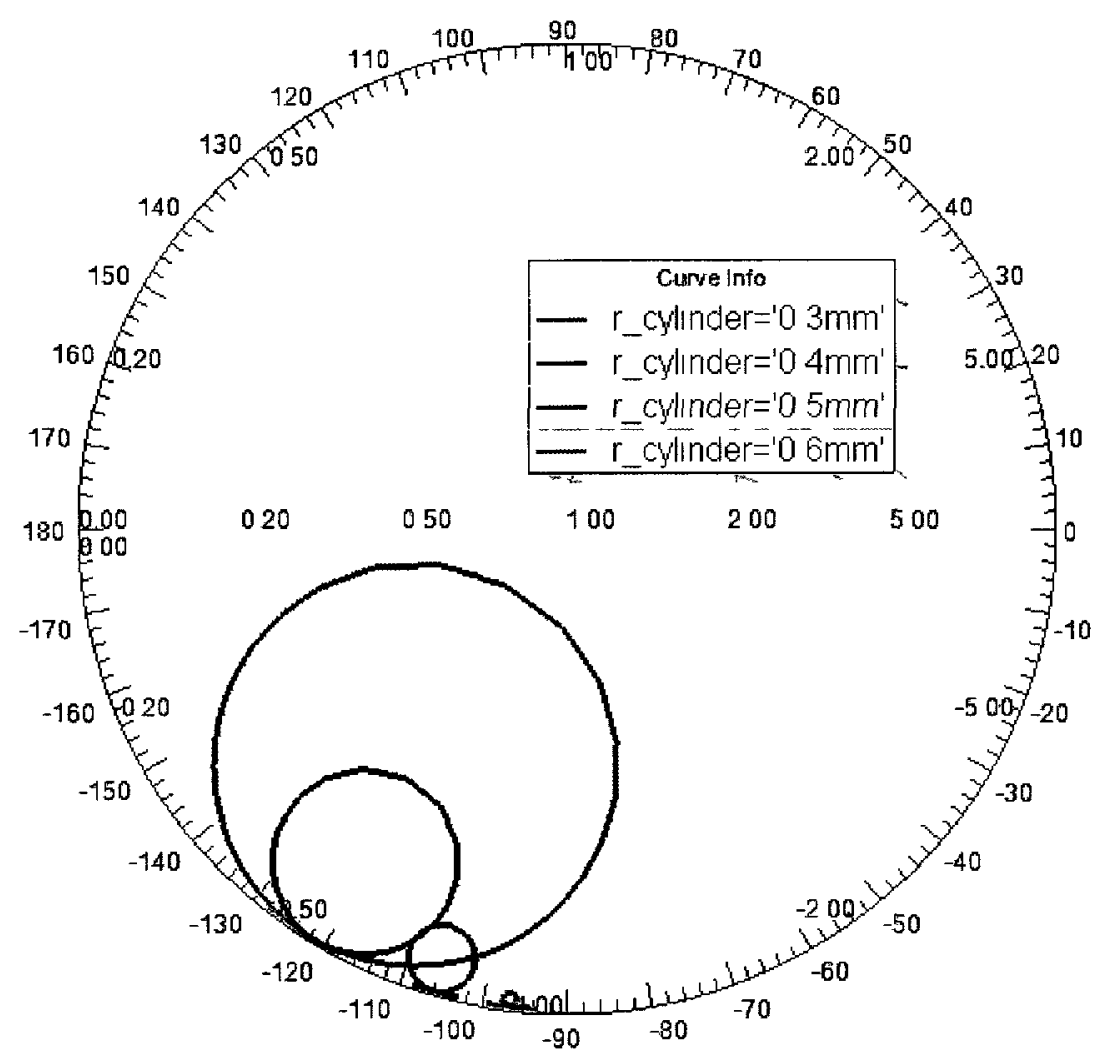

Figure 3-13: Simulated $S_{11}$ showing undercoupling for hemispherical cavity resonator

Figure 3-14 illustrates the trend of the simulated and calculated loaded quality factor versus aperture radius. Both calculated and simulated values agree with each other and have the same trend, although the calculated values are seemingly lower than the simulated values. This can be due to a number of factors. First of all the frequency in both cases is not identical. The calculations assumed a precise frequency of $20 \mathrm{GHz}$, while the simulations of the loaded hemispherical resonator present a resonant frequency at $20.26 \mathrm{GHz}$. This frequency shift is due to some assumptions that were made when designing the cavity, for example, the $3 \lambda / 4$ stub past the coupling aperture was designed at a frequency of $20 \mathrm{GHz}$ and is therefore assumed to have perfect dimensions. Calculations will take into account the perfect stub, whereas the simulations do not due to the shifted frequency. This causes the calculations to be misleading since they overestimate the coupling. Simulated coupling is expected to be weaker, resulting in a higher loaded quality factor with respect to the calculated quality factor. 


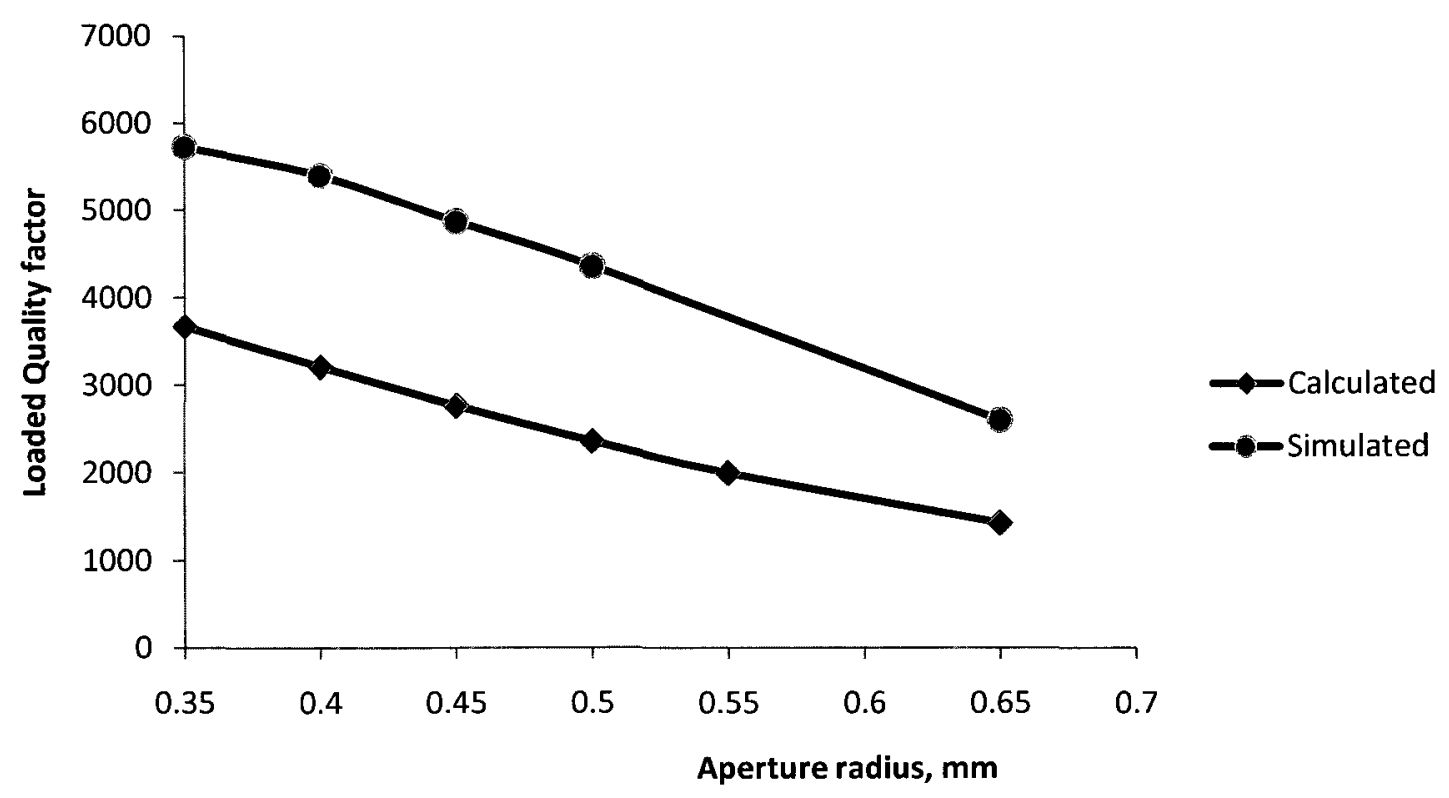

Figure 3-14: Calculated $\mathrm{Q}_{\mathrm{L}}$ vs. Simulated $\mathrm{Q}_{\mathrm{L}}$ as a function of aperture radius.

In addition, a wideband analysis with an aperture radius of $0.6 \mathrm{~mm}$ was performed to visualize the complete spectrum as shown in Figure 3-15. The first resonant frequency is at $20.26 \mathrm{GHz}$ with a return loss of $-13.24 \mathrm{~dB}$ and represents the $\mathrm{TM}_{011}$ mode. The resonant frequencies that follow are $28.53 \mathrm{GHz}$ with a return loss of $-12.7 \mathrm{~dB}$, representing the $\mathrm{TM}_{021}$ mode, $33.16 \mathrm{GHz}$ with a return loss of $14.7 \mathrm{~dB}$, representing the $\mathrm{TE}_{011}$ mode and $36.68 \mathrm{GHz}$ with a return loss of $-10 \mathrm{~dB}$, representing the $\mathrm{TM}_{031}$ mode, for $f_{l}, f_{2}$ and $f_{3}$ respectively. As discussed in Section 3.3, the higher resonant frequencies observed are due to the higher order modes present in the cavity and seem to follow the calculated values quite accurately. 


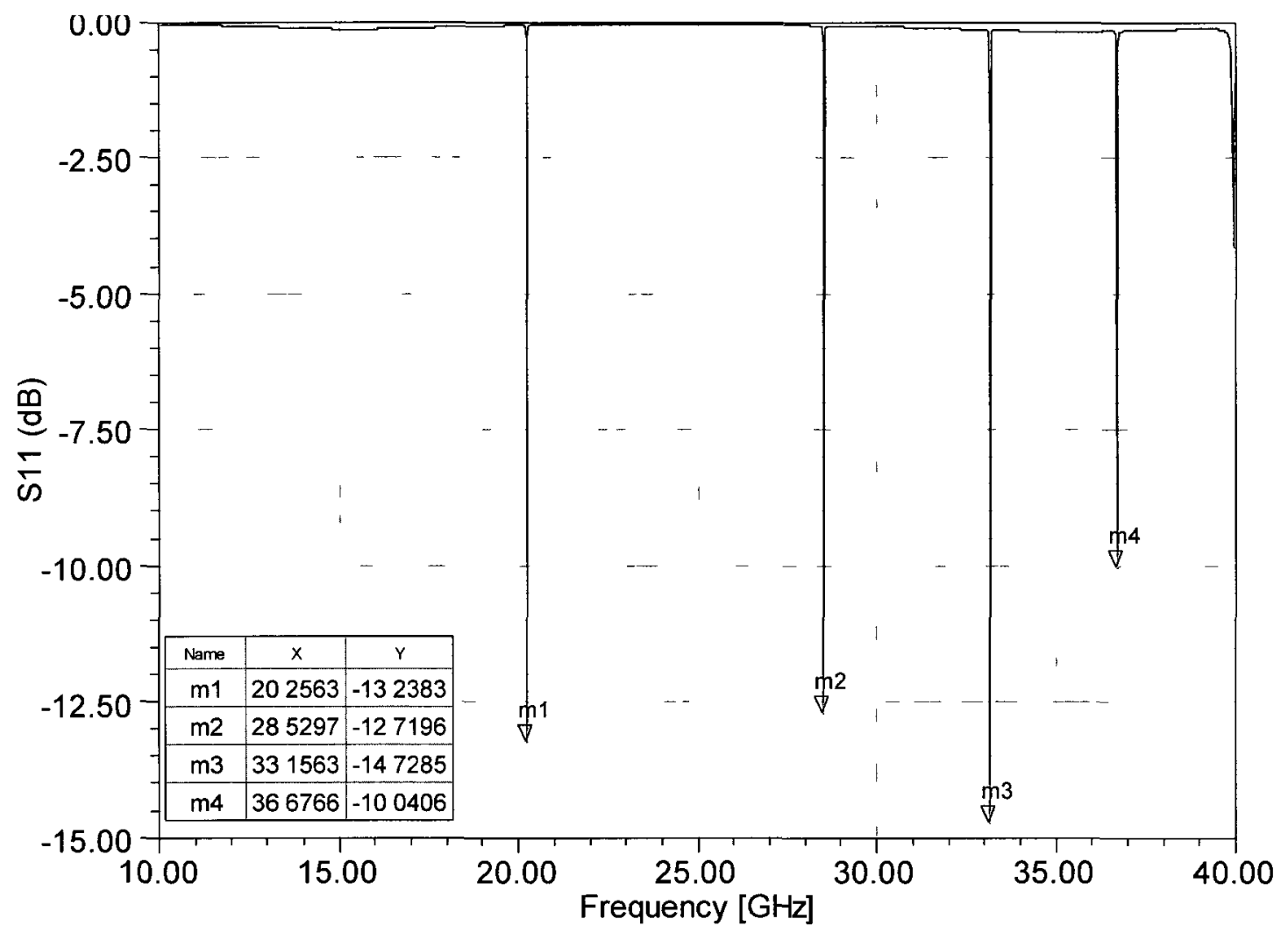

Figure 3-15: Wideband spectrum for an aperture radius of $0.6 \mathrm{~mm}$. 


\section{Chapter 4}

\section{Resonator Study}

The hemispherical cavity resonator design discussed in chapter 3 will be further analyzed in this chapter. The parameters which affect the resonance and especially the quality factor of the resonator will be studied.

\subsection{Sensitivity Analysis}

The performance and quality factor of the hemispherical cavity resonator highly depend on many of the parameters previously discussed in chapter 3 , such as aperture radius, aperture location and dimension of the feeding mechanism. An in depth analysis will be performed on how these parameters affect the loaded quality factor of the resonator. This analysis is valuable especially when one of the key goals is to optimize the quality factor of the resonator.

The measurement of the quality factor is not immediate and its value is not read off of the simulation tool as for the S-parameters. Unfortunately, a simple method for the measurement of $\mathrm{Q}_{\mathrm{u}}$ and $\mathrm{Q}_{\mathrm{L}}$ is not available, although literature provides various ways of determining the quality factor through the S-parameters of one-port systems. Some include the $3 \mathrm{~dB}$ bandwidth method [31], the Critical-Points Method [32] and the standard three points method [33,34] along with many others [35-37]. Unfortunately, the first 
method i.e. the $3 \mathrm{~dB}$ bandwidth method has the disadvantage of using only three frequency points to measure the loaded, unloaded and external quality factors, even though the frequency sweep can contain hundreds of data points [38]. The Critical-Points method has proved to be quite effective but it is a graphical method and is more open for errors. The standard three points method results in the most effective and accurate for our applications [39] and is chosen here. This method is derived from the "Q-circle" approach [40] and is embodied in the computer program QZERO MATLAB for windows $[41,42]$. This program makes use of a one-port S-parameter file at many frequencies. The program interpolates the data to obtain the center and diameter of the $\mathrm{Q}$ circle. It then interpolates the three frequency points required to extract the values of loaded and unloaded quality factor, resonant frequency and coupling coefficient. The user can choose the high frequency point and the low frequency points used in the interpolation. The program has proven to be very sensitive to the high and low frequency points that are chosen.

The following parameters have been studied and some typical values of the tolerances correspond to those of the Duroid manufacturing facility:

- Aperture Radius;

- Movement of aperture in $\pm x$ direction ( $\pm 1 \mathrm{mil}$ );

- Position of aperture in $\pm y$ direction $( \pm 0.5 \mathrm{~mm})$;

- Width of the microstrip feed line ( $\pm 1 \mathrm{mil})$;

- Offset of the microstrip feed line from the centre of the cavity $( \pm 1 \mathrm{mil})$;

The sensitivity analysis for the aperture radius can be found in Section 3.6. In fact, Figure 3-12 clearly shows that changing the aperture radius can seriously degrade the performance of the cavity resonator. Specifications clearly state that the quality factor of the resonator should be greater than 2000 (Table 1-1). This specification would not be met if an aperture radius of $0.65 \mathrm{~mm}$ or greater was used, therefore making this one of the most critical parameters in order to meet the required specifications.

The position of the centre of the aperture in the $\pm x$ direction by \pm 1 mil was also studied. When analyzing the manufactured PCBs, alignment marks were made in order to secure proper positioning of the aperture. This of course, comes along with its own 
tolerances due to the fact that the alignment marks are hand-made. Although this was done, there have been some issues, further examined in the following sections, which have suggested that this is one of the most important parameters to study. S-parameter data concludes that $\mathrm{a} \pm 1 \mathrm{mil}$ difference in the position of the aperture does not present a significant variation in frequency or in magnitude, as shown in Figure 4-1 and that it also does not significantly affect the quality factor of the resonator, as shown in Figure 4-2.

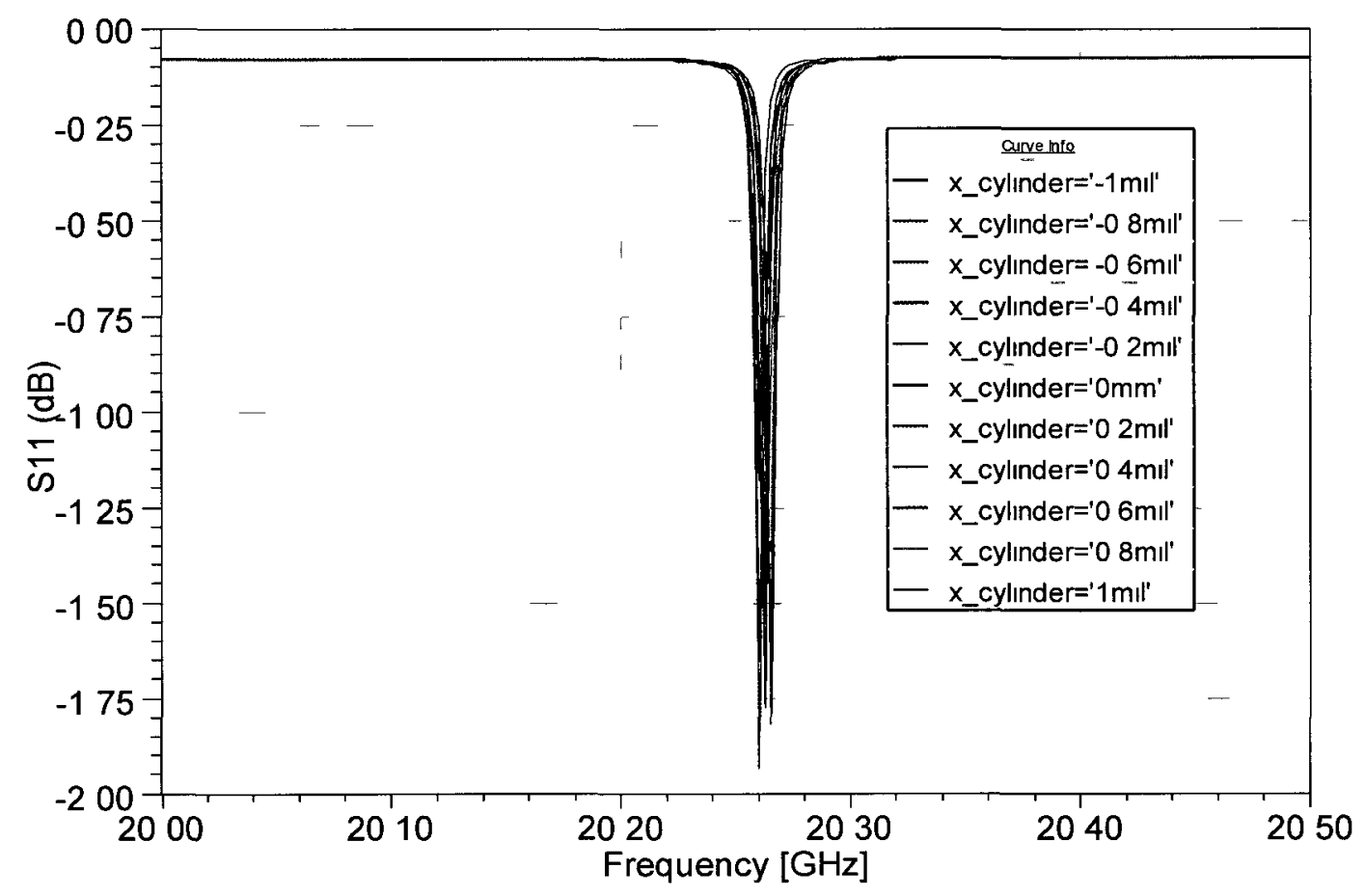

Figure 4-1: Return loss vs. frequency for aperture movement in $\pm x$ direction by $\pm 1 \mathrm{mil}$. 


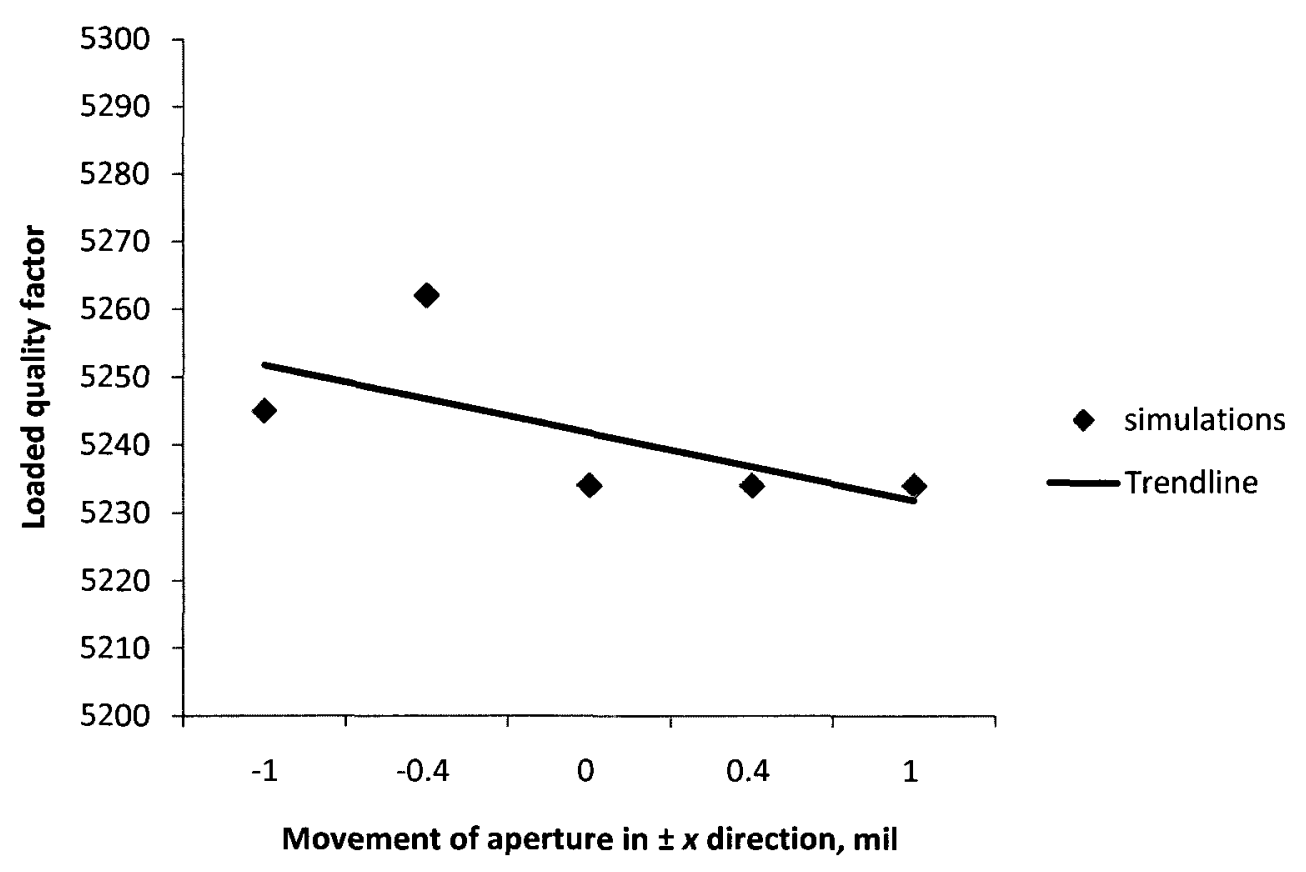

Figure 4-2: Movement of aperture in $\pm x$ direction vs. loaded quality factor

Similarly to the movement of the aperture in the $\pm x$ direction, the position of the aperture in the $\pm y$ direction was also studied. The position of the aperture was originally calculated to be $5 \mathrm{~mm}$ from the centre of the hemispherical cavity (centre to centre), from Equation 3.4-6, but sensitivity analyses are critical as the position can shift easily when the PCB card is positioned in the jig. Instead of analyzing the movement of the aperture by \pm 1 mil, as in the previous case, the position is adjusted by $\pm 0.5 \mathrm{~mm}$ in order to see if a greater gap would affect the quality factor. S-parameter data, as shown in Figure 4-3, suggests that there is no major change in the magnitude of $S_{11}$ or in the frequency for aperture positions varying from $4.5 \mathrm{~mm}$ to $5.5 \mathrm{~mm}$. With the QZERO program, this Sparameter data provided values for loaded quality factor as shown in Figure 4-4. Although there are some slight deviations in the loaded quality factor, it seems to be fairly constant in the region between $4.5 \mathrm{~mm}$ and $5.5 \mathrm{~mm}$. 


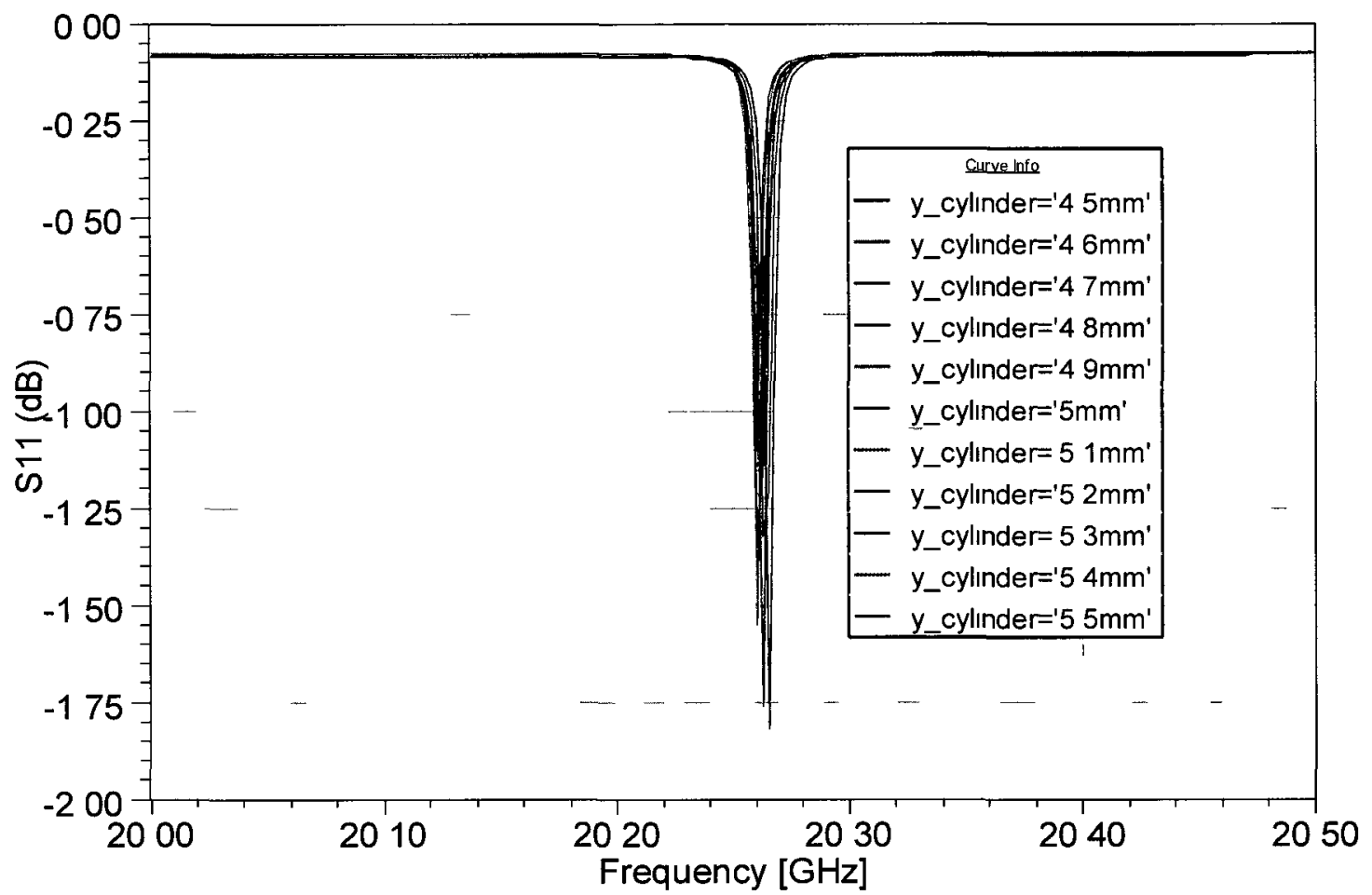

Figure 4-3: Return loss vs. frequency for aperture position in $\pm y$ direction by $\pm 05 \mathrm{~mm}$

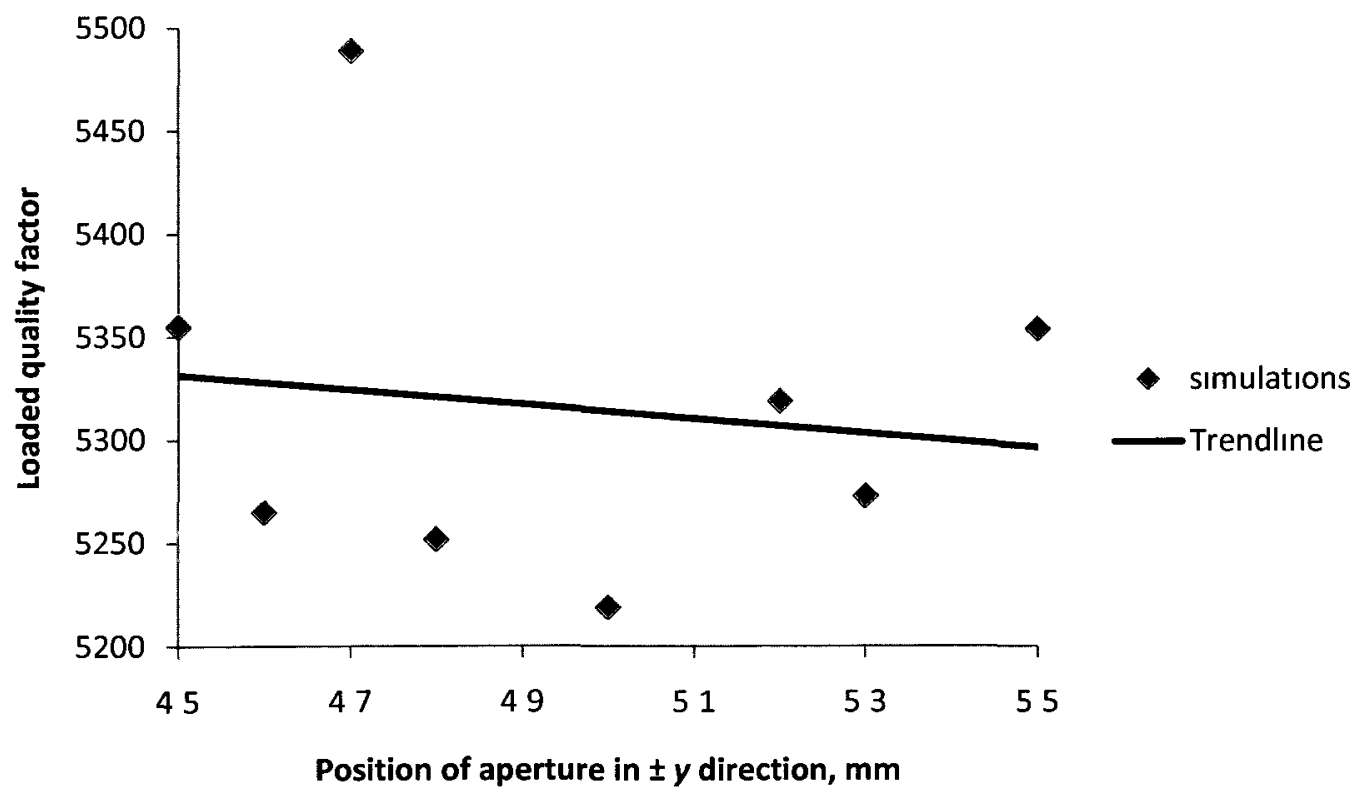

Figure 4-4: Position of the aperture in $\pm y$ direction vs. loaded quality factor 
The width of the microstrip feed line was determined to be $763.62 \mu \mathrm{m}$, as shown and described in section 3.5. It is important to analyze the sensitivity of the width of the microstrip by at least \pm 1 mil to ensure that over-etching or under-etching is taken into account and therefore that no disastrous results are observed when measurements are taken. S-parameter data shows no major change in the magnitude of $\mathrm{S}_{11}$ or in the frequency, as shown in Figure 4-5. Loaded quality factors were also simulated from QZERO, shown in Figure 4-6, and proved to be fairly constant for variations of \pm 1 mil.

A question that arose during the sensitivity analysis of the resonator was what would happen if the microstrip feed was not perfectly aligned with the centre of the hemispherical cavity as it is in Figure 4-7. The difference from the centre of the cavity to the centre of the microstrip feed is called the offset and it was investigated to see if the loaded quality factor would change drastically if the feed was not positioned correctly. Figure 4-8 shows the simulations of the magnitude of $S_{11}$ for an offset of \pm 1 mil in the $x$ direction, done in HFSS. As can be seen, there is no drastic or significant change in the frequency or in the magnitude of $S_{11}$.

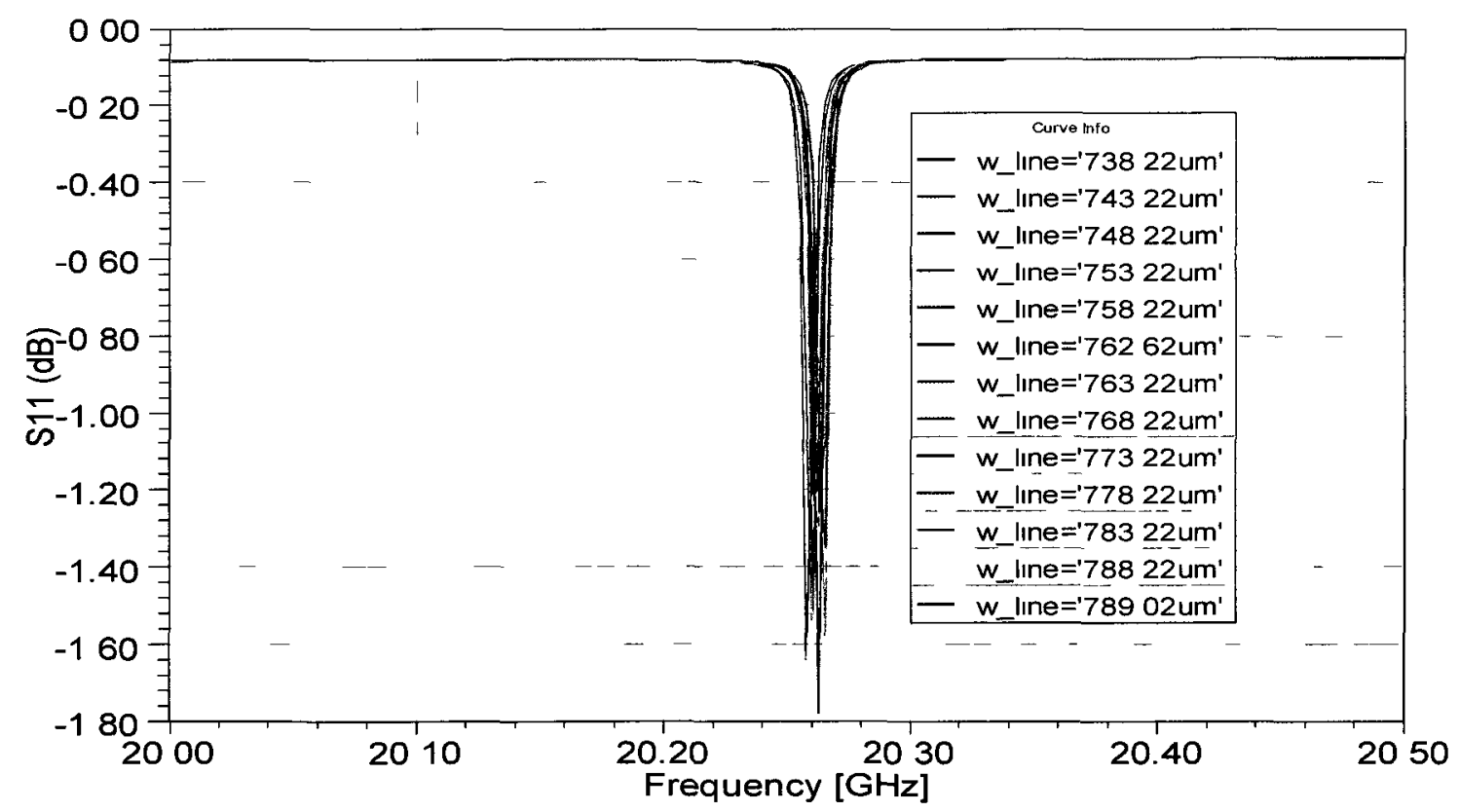

Figure 4-5: Return loss vs. frequency for variable microstrip line width 


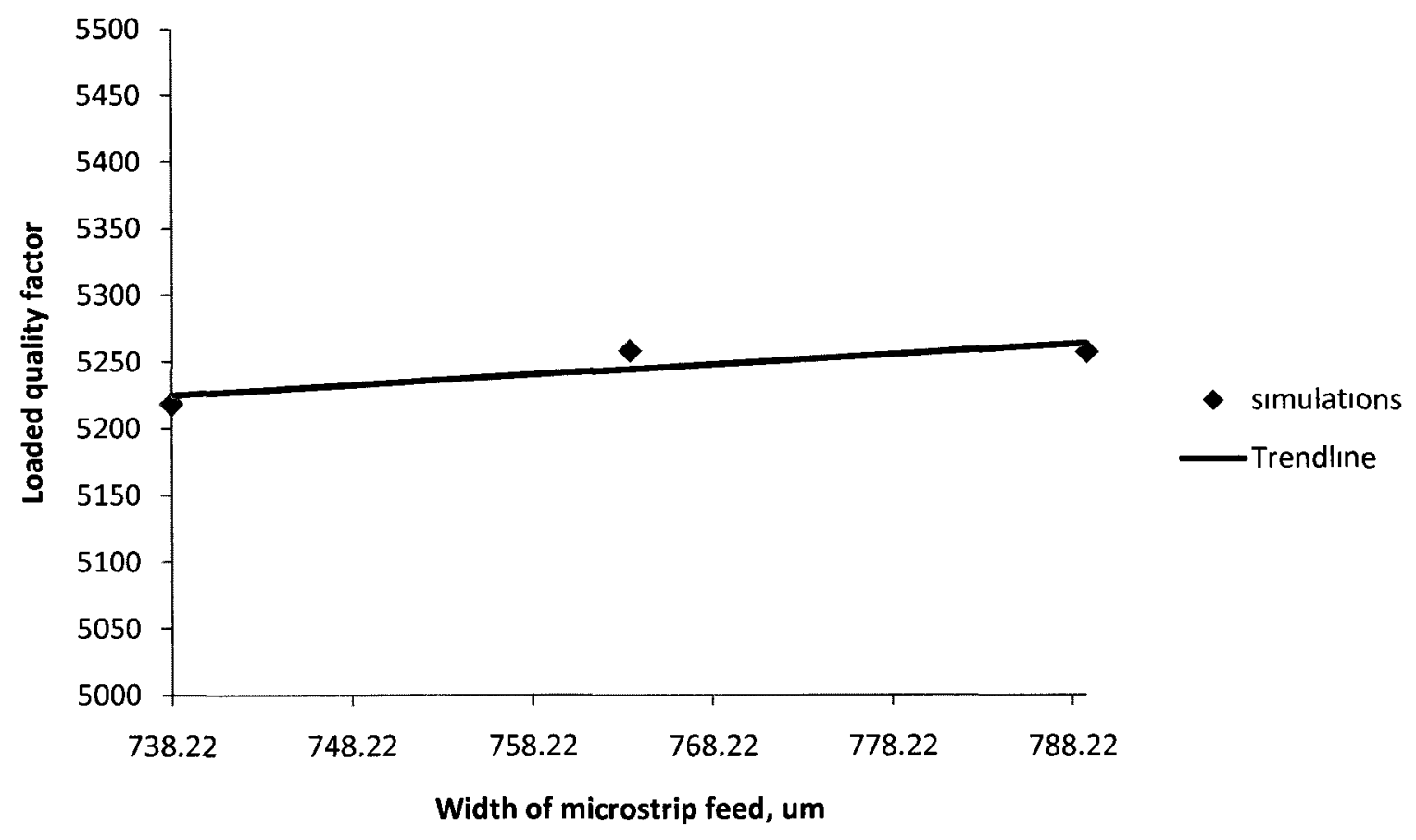

Figure 4-6: Width of microstrip feed line vs. loaded quality factor

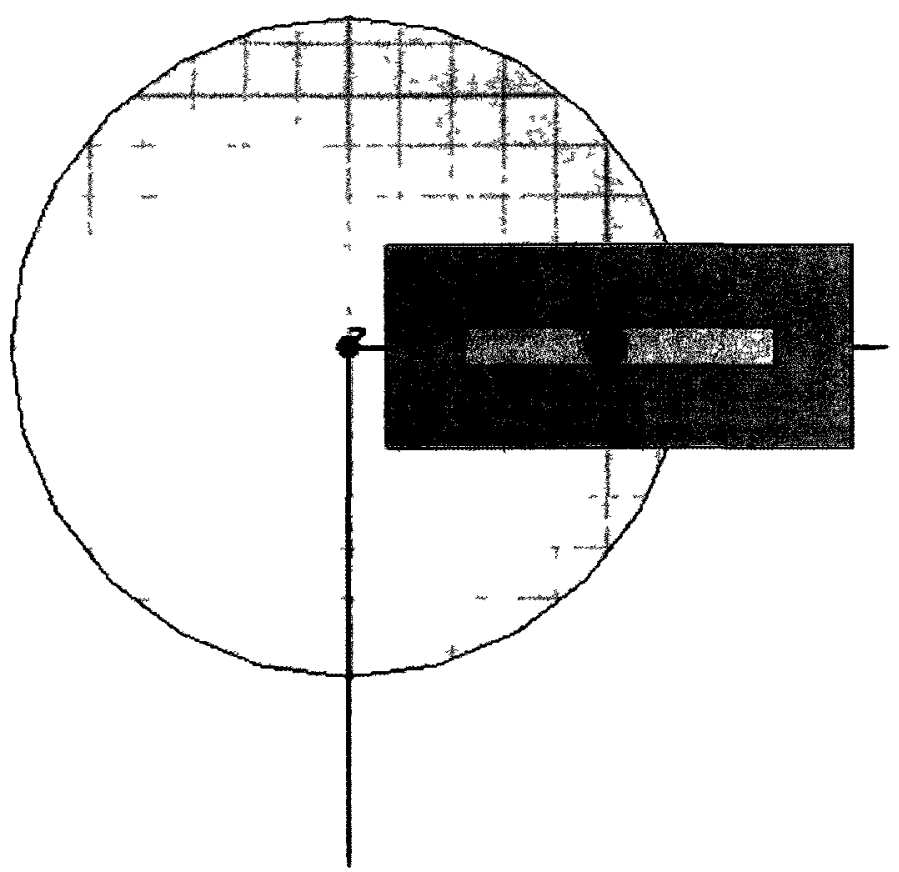

Figure 4-7: Top view of cavity resonator 


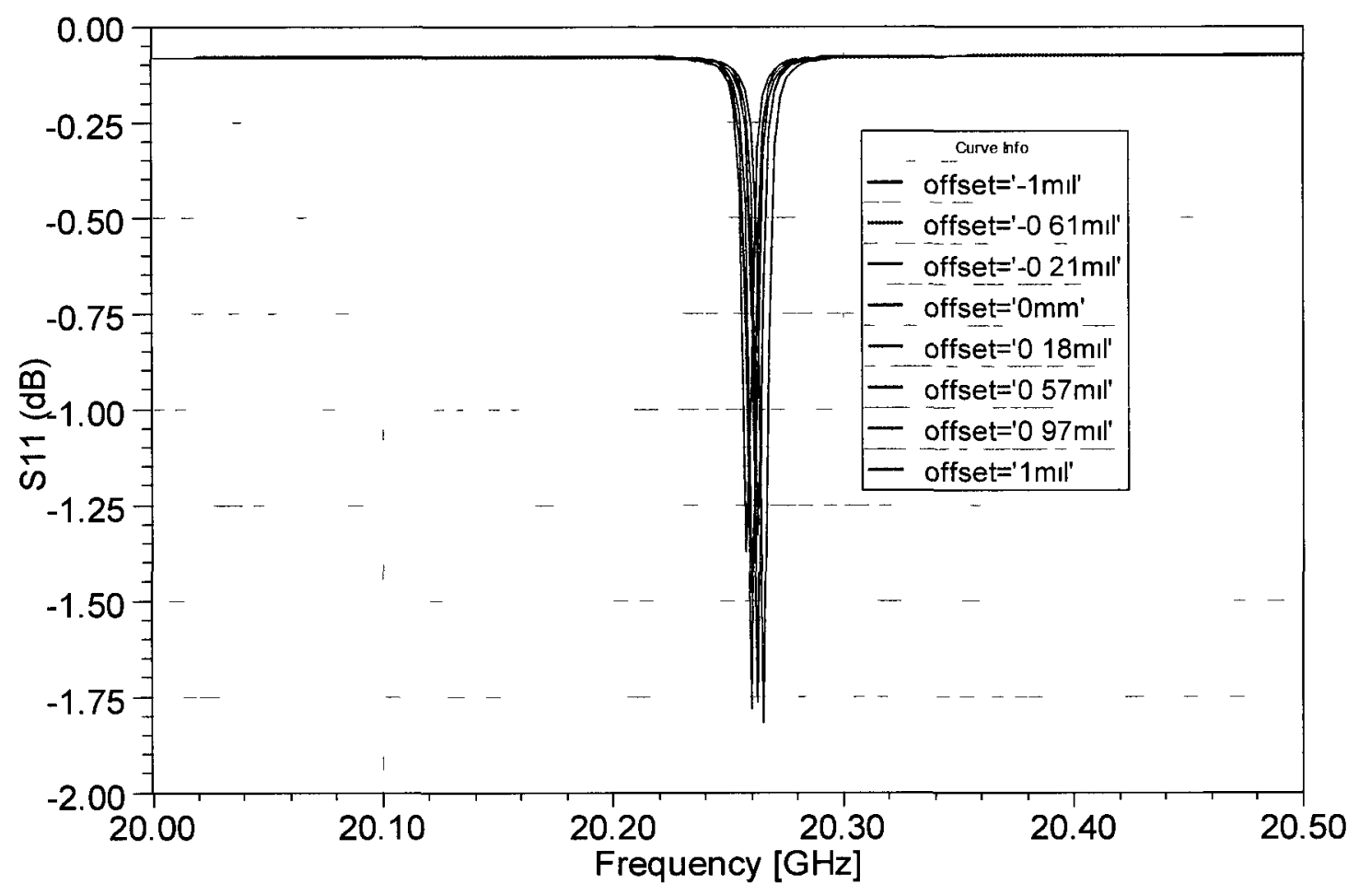

Figure 4-8: Return loss vs. frequency for variable offset

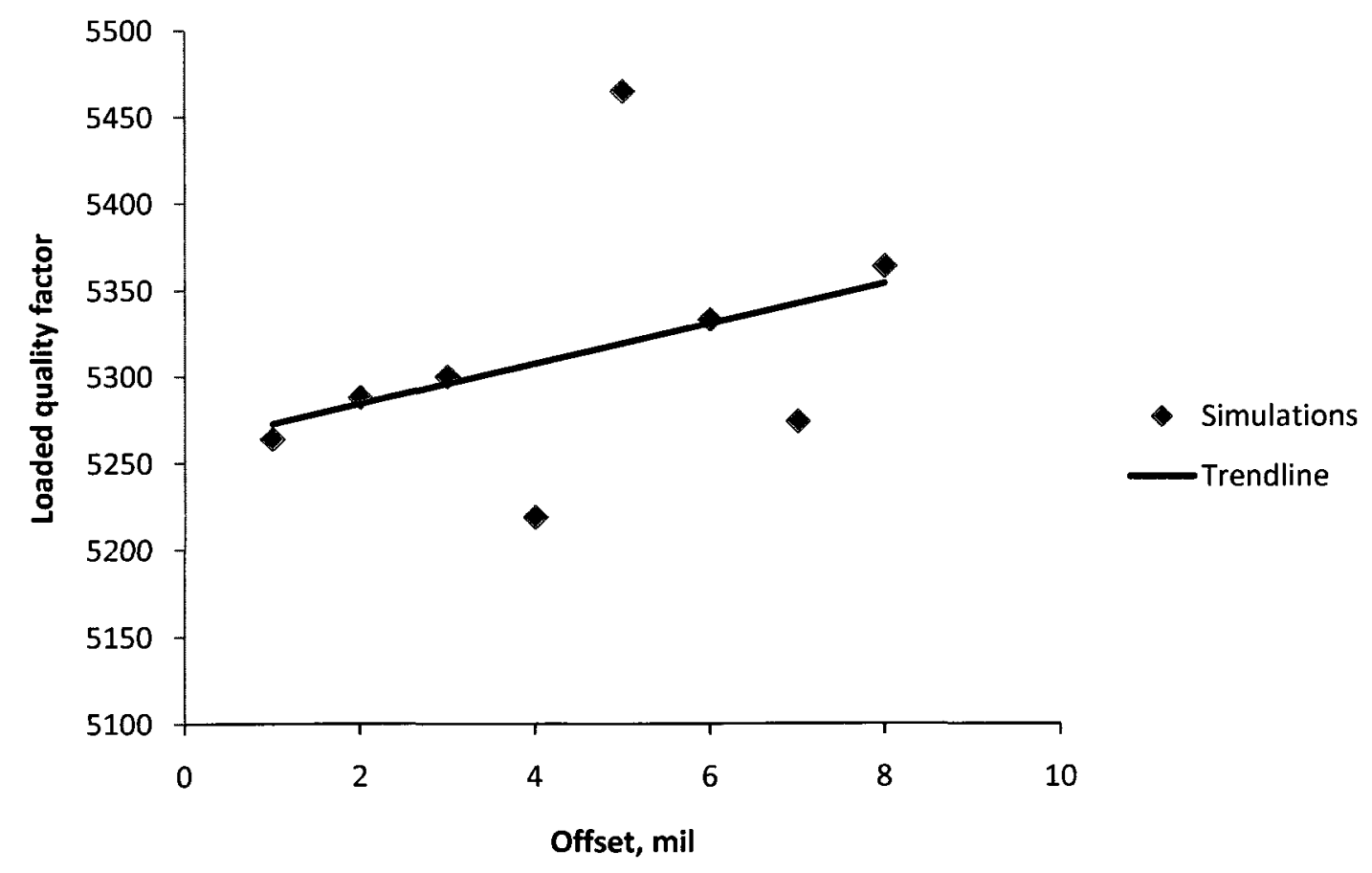

Figure 4-9: Offset vs. loaded quality factor 
The loaded quality factor was also plotted versus the offset and although there are some minor differences, a \pm 1 mil change in the position of the microstrip feed in the $\pm x$ direction will have no major effect on the loaded quality factor and therefore on the performance of the resonator.

\subsection{Bond Wire Analysis}

Bond wires are the most common way of interconnecting microwave ICs thanks to their low cost, flexibility and good return loss performance. The characterization and modeling of bond wires is an important factor to take into account for high frequency applications, as it is the high impedance of the bond wire that causes reflections and inductive discontinuities. Although there has been a lot of research in the modeling and experimentation of bond wires for frequencies up to $100 \mathrm{GHz}$ [43] it is interesting to see the effects of the bond wires specific to our structure and to our frequency.

A simple structure was implemented in HFSS for a typical wire bond and for a ribbon bond, as shown in Figure 4-10 and 4-11 respectively, in order to study the return loss of the bond wire and therefore to determine if it would present a significant degradation in performance. 
a)

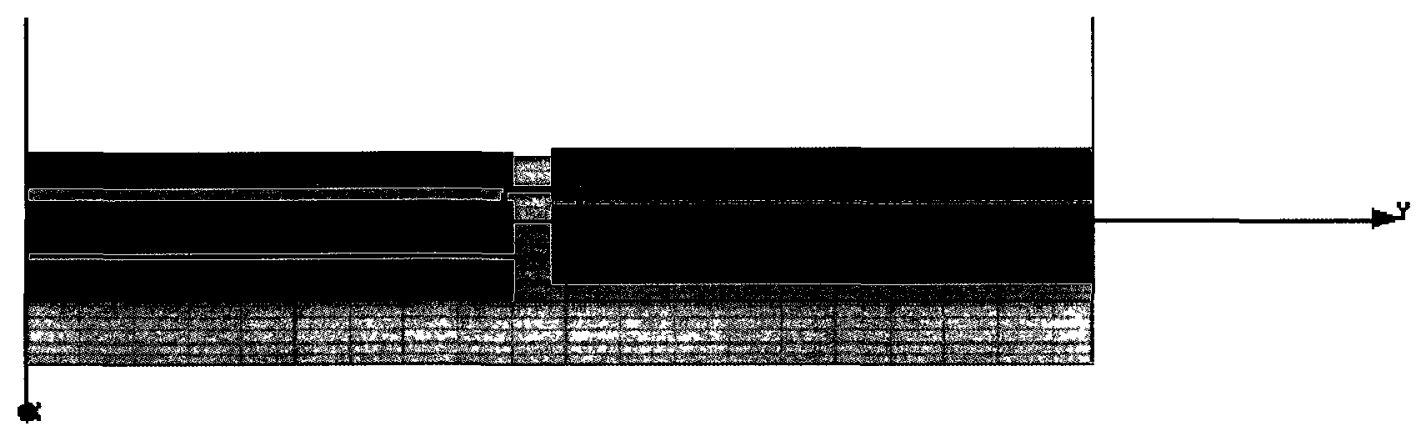

b)

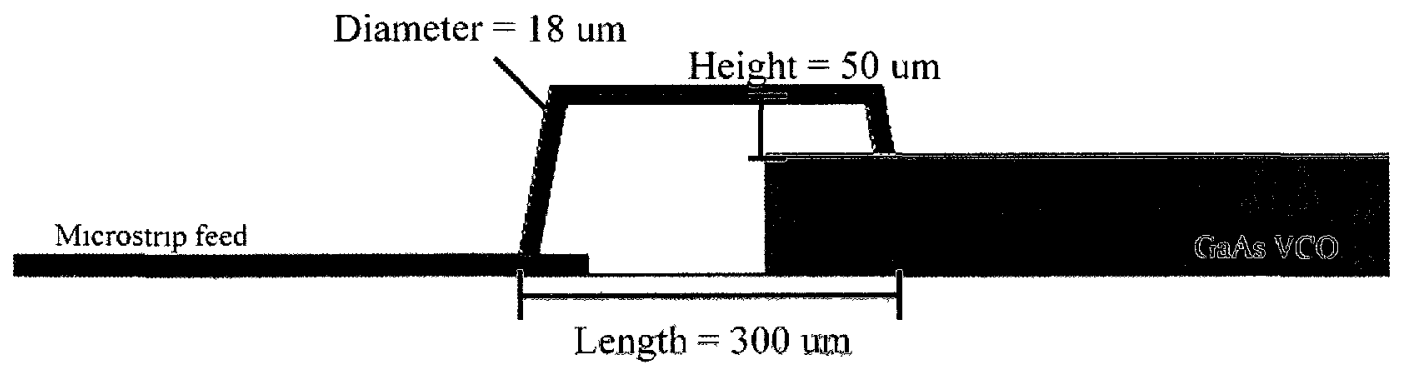

Durotd 5880

Figure 4-10: Wire bond setup a) 3D view, b) side zoom of wire bond 
a)

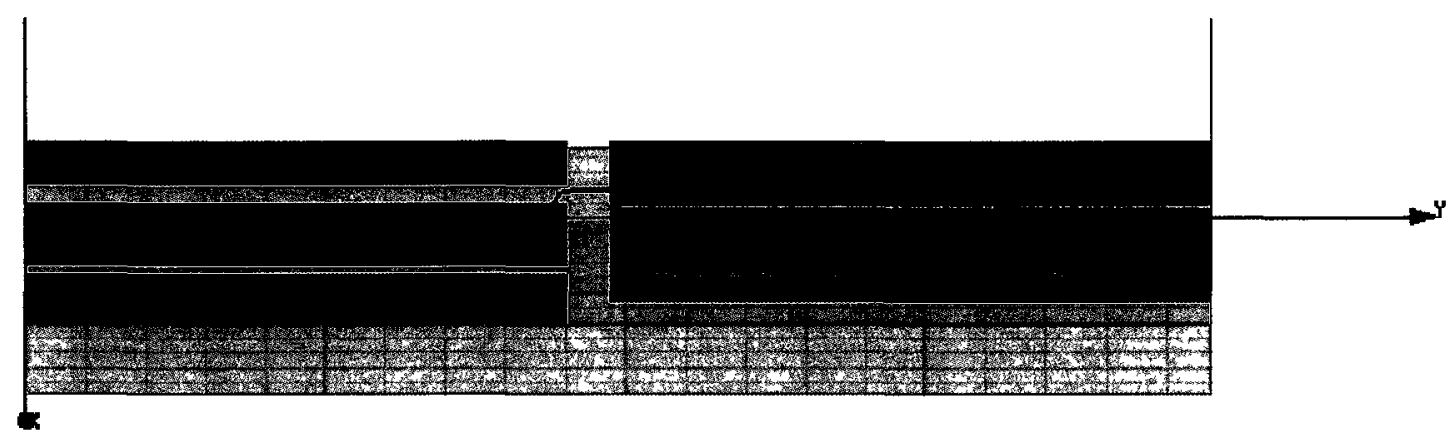

b)

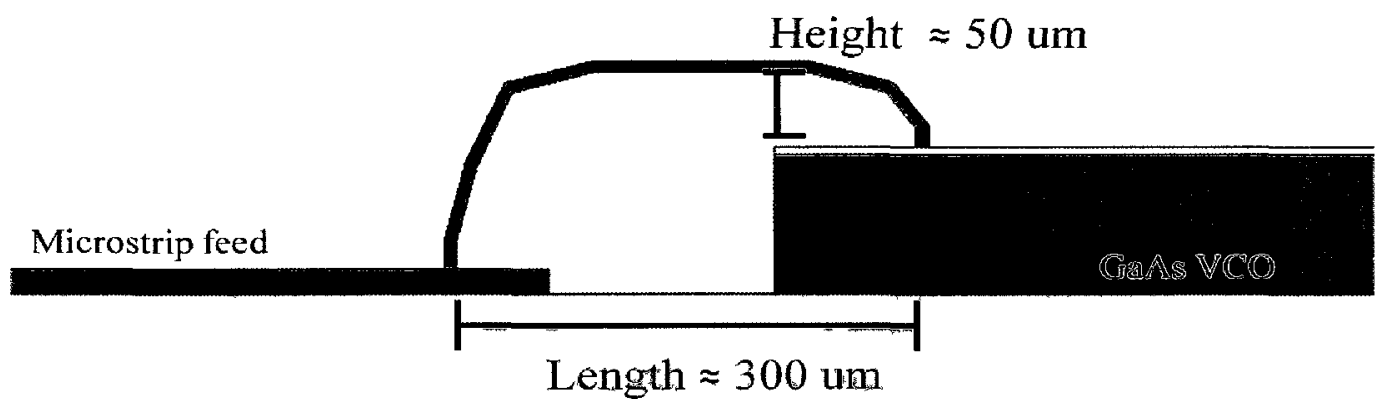

Duroid 5880

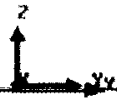

Figure 4-11: Ribbon bond setup a) 3D view, b) side zoom of ribbon bond

Both bonds are made of $99.99 \%$ gold but are of different sizes. The wire bond is $0.0007 "$, while the ribbon bond is approximately $0.0005 " \mathrm{x} 0.0003$ " since it was created manually to best resemble the ribbon shape. The bond that was analyzed is the one connecting the microstrip of the resonator to a pad on the GaAs VCO chip. Proper de-embedding was also taken care of in HFSS. Figure 4-12 shows S-parameter data for the wire bond, collected from HFSS, with relative data at $20 \mathrm{GHz}$. 


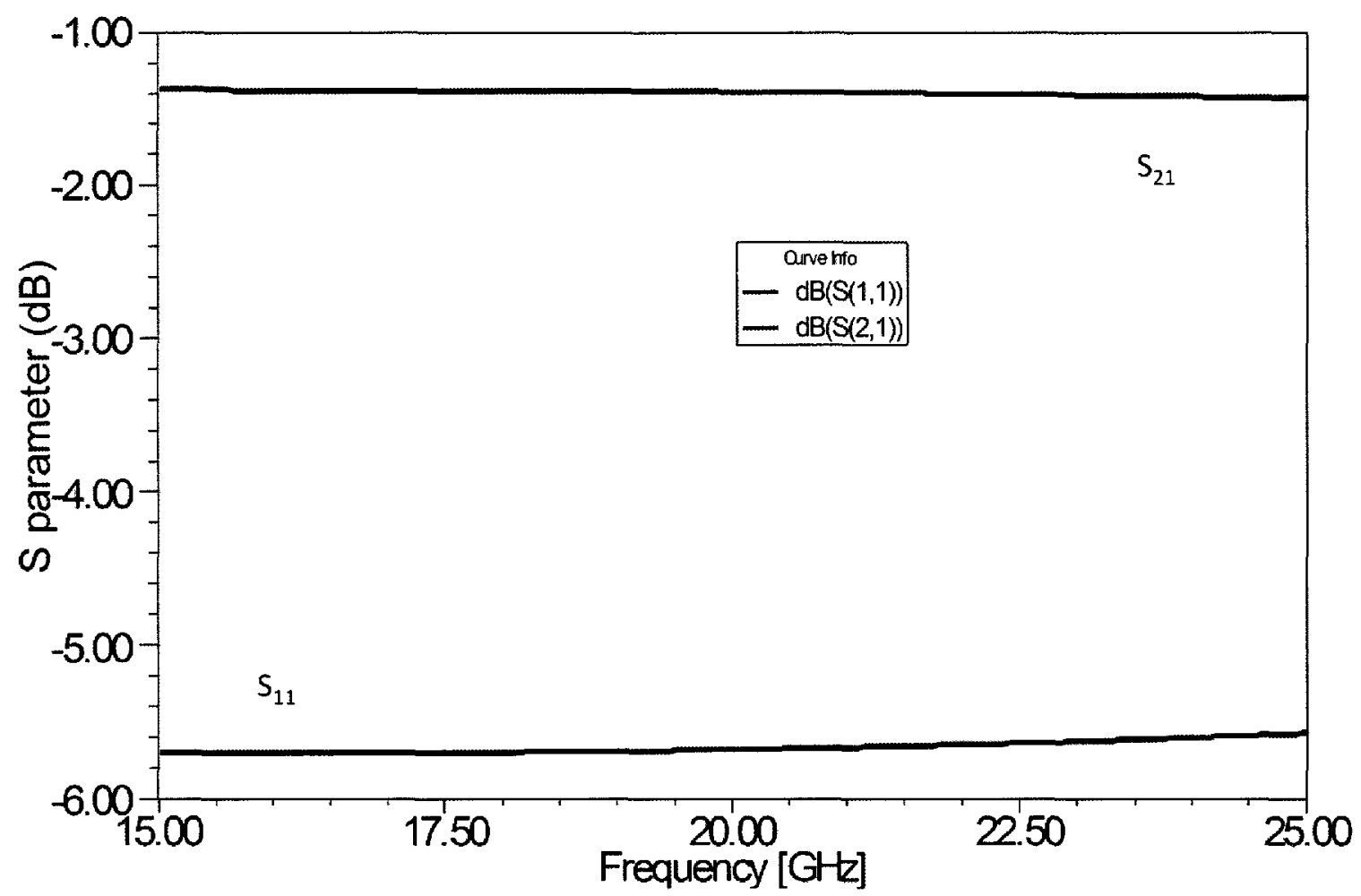

\begin{tabular}{|c|c|c|}
\hline @ $20 \mathrm{GHz}$ & $\mathbf{S}_{\mathbf{1}}$ (Mag/Phase (deg)) & $\mathbf{S}_{\mathbf{2}}$ (Mag/Phase (deg)) \\
\hline $\mathbf{S}_{\mathbf{1}}$ (Mag/Phase (deg)) & $(0.52,-10.5)$ & $(0.85,-22.1)$ \\
\hline $\mathbf{S}_{\mathbf{2}}$ (Mag/Phase (deg)) & $(0.85,-22.1)$ & $(0.51,146)$ \\
\hline
\end{tabular}

Figure 4-12: S-parameter data for wire bond.

The insertion loss is therefore:

$$
I L=20 \log \left|S_{21}\right|=-1.4 d B
$$

A parametric analysis was also conducted for the effects of the length of the wire bond on the insertion loss. Figure 4-13 presents the trend of the insertion loss versus the length of the wire bond at a frequency of $20 \mathrm{GHz}$. As the length increases, the insertion loss also increases due to the increased inductance and loss in the wire bond. 


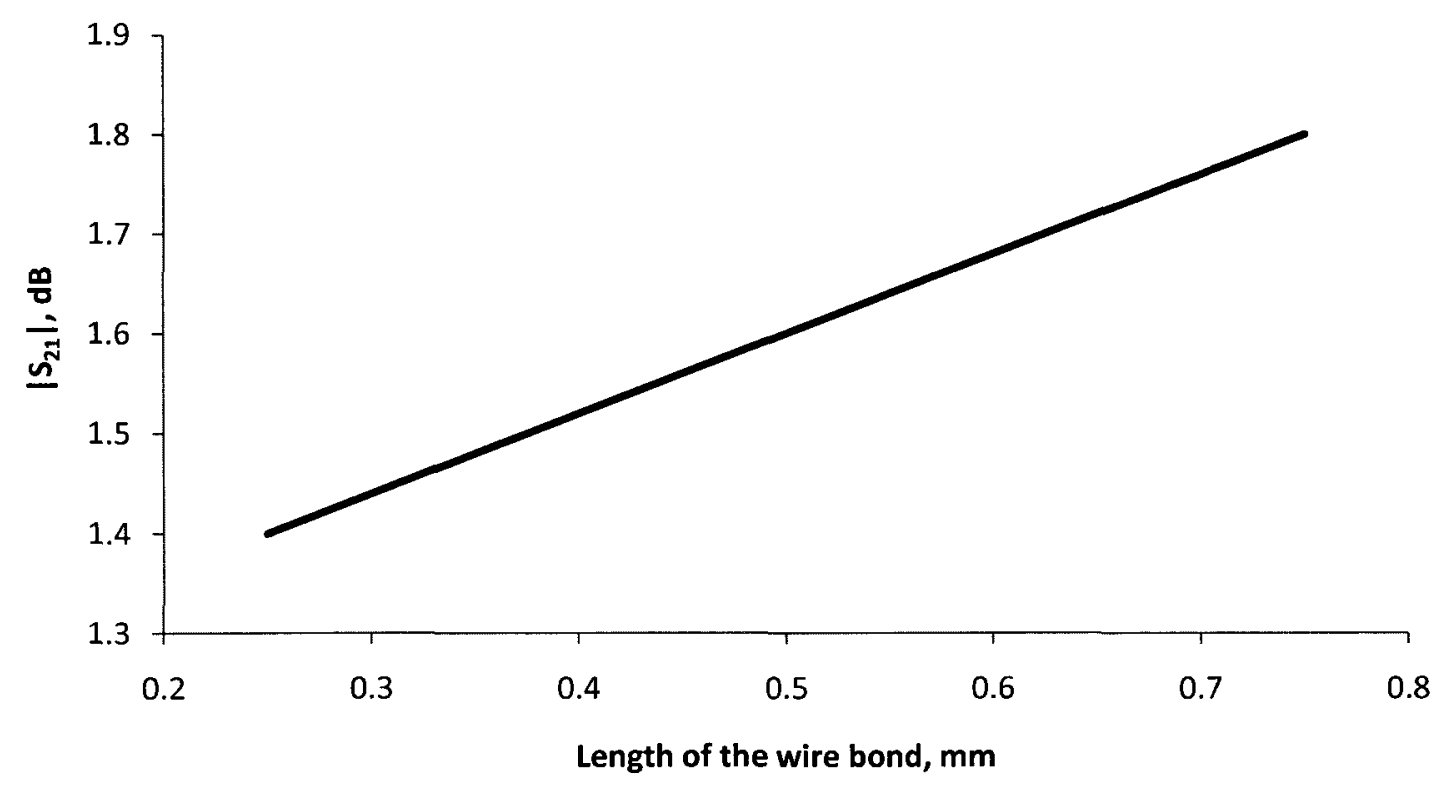

Figure 4-13: Length of wire bond vs. $\left|\mathrm{S}_{21}\right|$

To determine the change in the quality factor of the resonator with the wire bond taken into consideration, the wire bond setup was added to the resonator design as shown in Figure 4-14. S-parameter data was extracted, Figure 4-15, and the quality factor was analyzed with QZERO. 


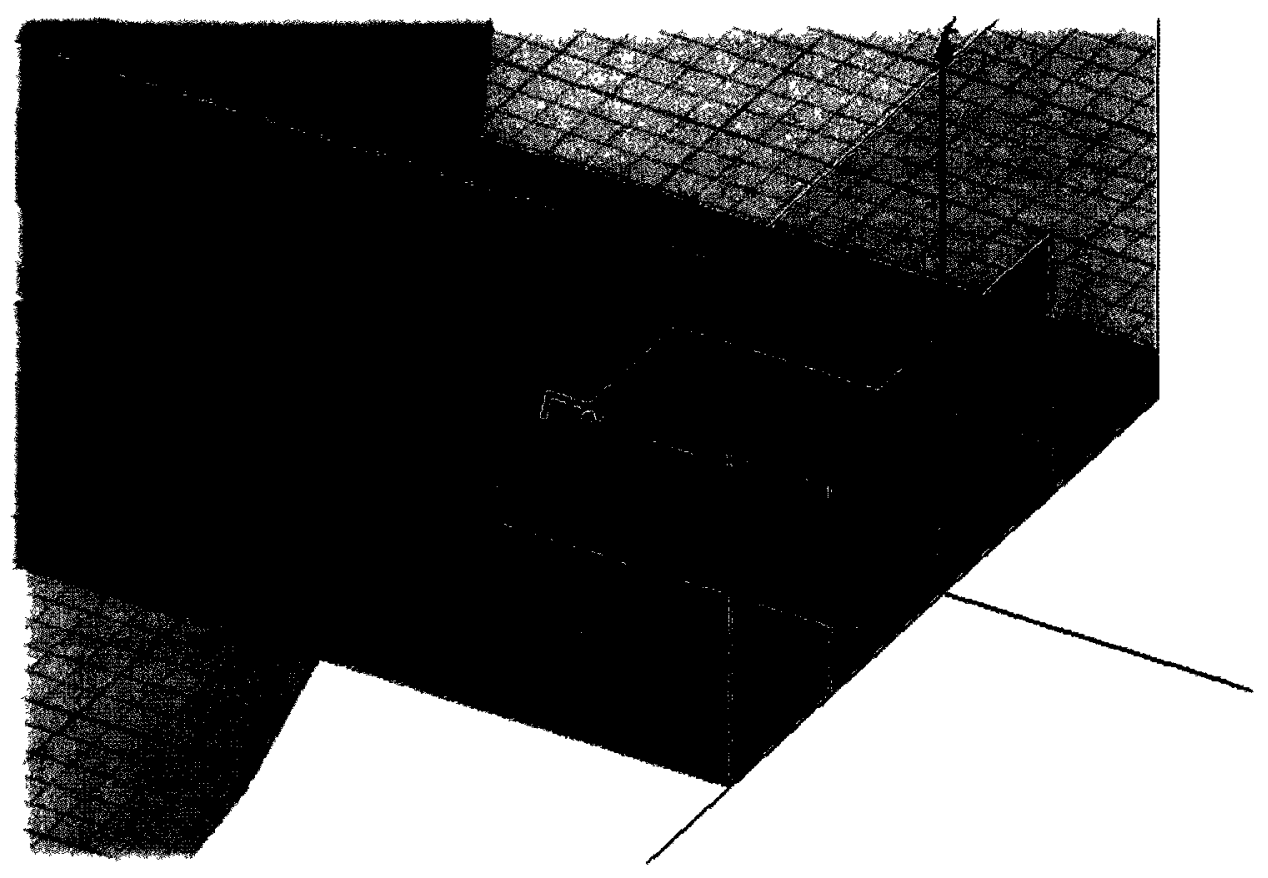

Figure 4-14: Wire bond and resonator setup

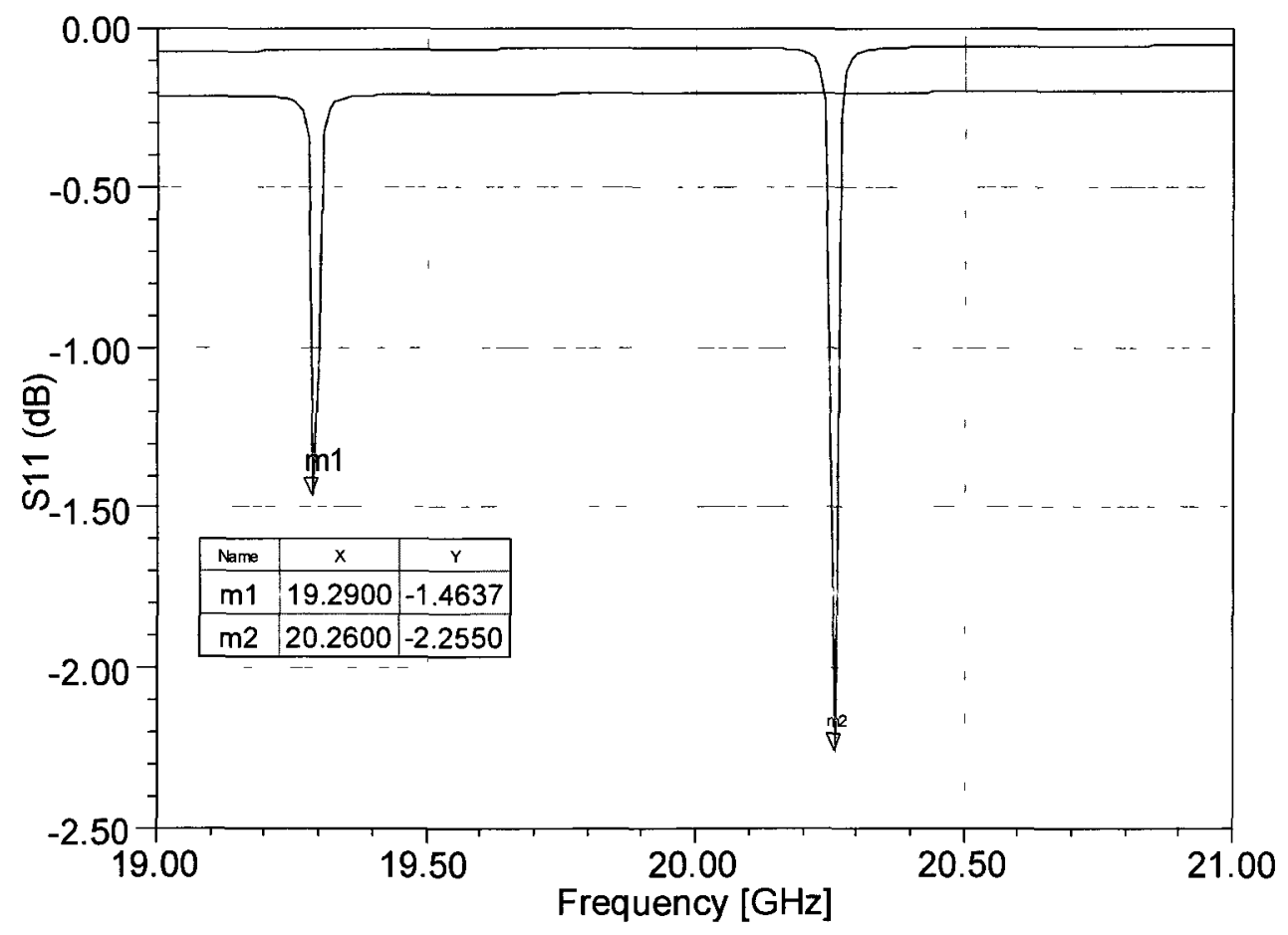

Figure 4-15: S-parameter data for wire bond and resonator setup, $\mathrm{ml}=$ with bond wire, $\mathrm{m} 2$ = without bond wire. 
The analysis of the wire bond presented a frequency shift as shown in Figure 4-15. This is due to the inductance and the parallel capacitances present in the wire bond. The unloaded and loaded quality factors at this frequency were constant and did not present a significant change. The same setup and procedure was completed for the ribbon bond and although it presented a slightly lower insertion loss, by about $0.1 \mathrm{~dB}$, it also did not alter the quality factor by a significant amount.

\subsection{RT/duroid® 5880 Microstrip Resonator}

It is interesting to consider how a simple microstrip resonator compares to the aperture coupled hemispherical cavity resonator pursued up to now. A simple $\lambda / 4$ short microstrip resonator in RT/duroid 5880, was implemented in the electronic design software ADS (Advanced Design System) from Agilent Technologies. Calculations were done to convert the $N / 4$ short microstrip resonator into its parallel lumped components with simple microwave resonator equations with results as shown in Figure 4-16.

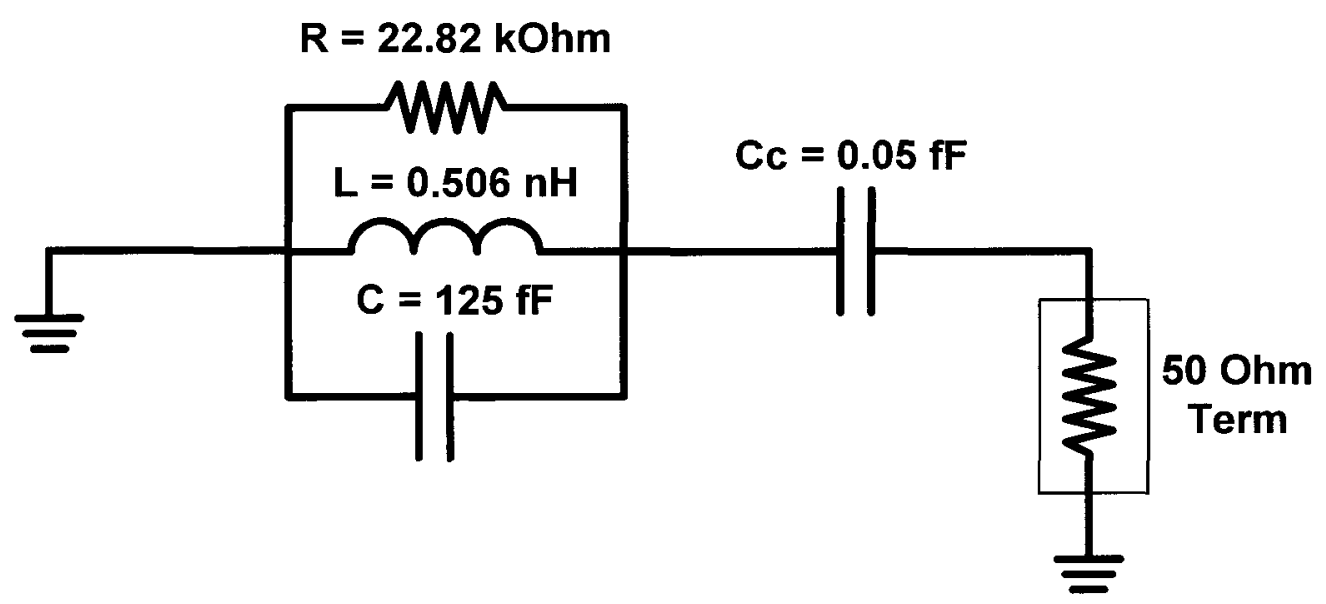

Figure 4-16: Circuit of parallel lumped $N 4$ short microstrip resonator. 
S-parameter data was collected in order to compare the loaded and unloaded quality factors of the resonator. Figure 4-17 shows S-parameter data and a resonant frequency of 19.96 GHz. This data was analyzed by QZERO, as was done with the hemispherical cavity resonator, and provided an unloaded quality factor of 360 and a loaded quality factor of 358 . This would obviously not be a high enough loaded quality factor for the purpose of this thesis and for the E-band oscillator proposed [7].

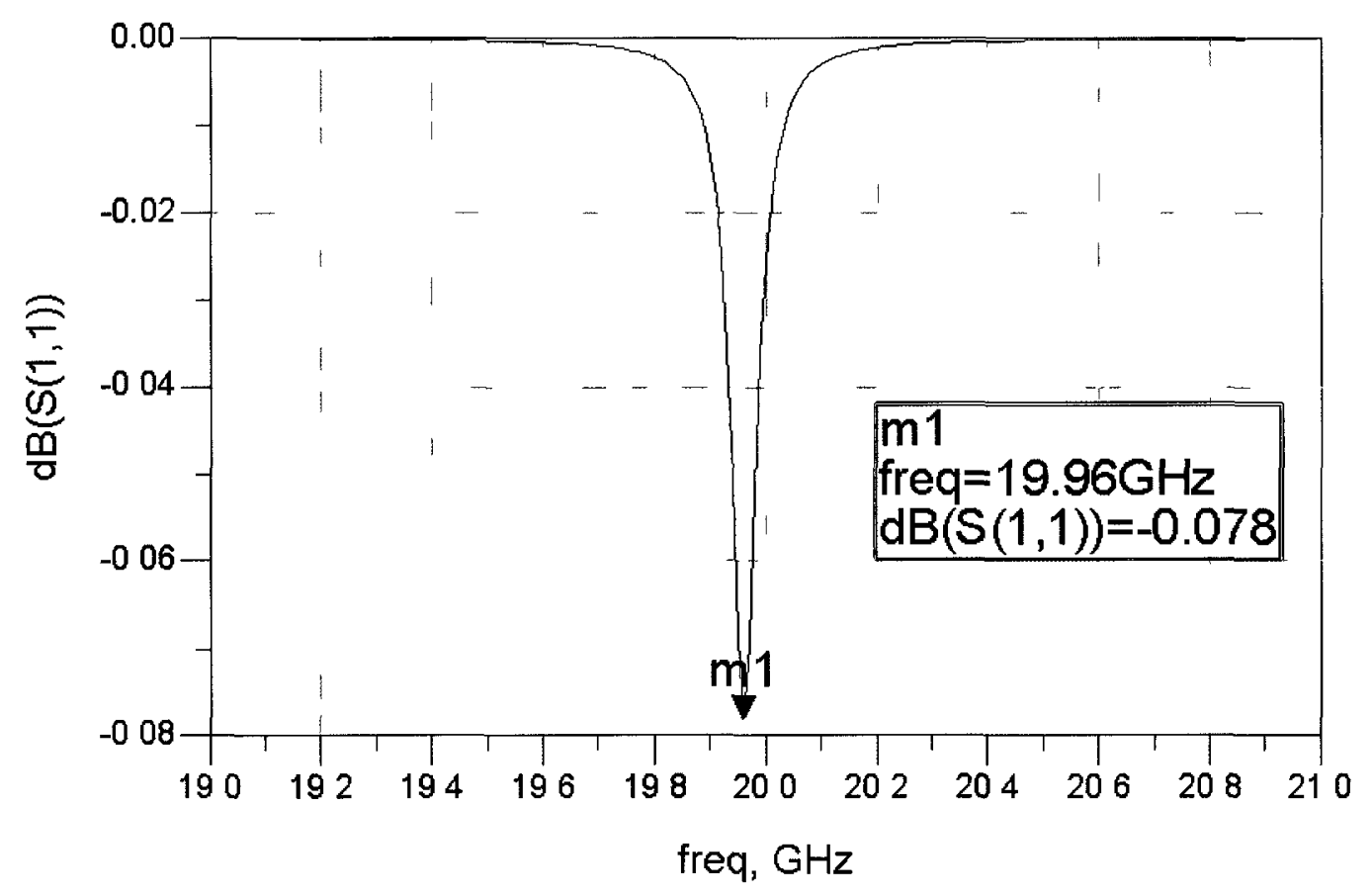

Figure 4-17: Resonant frequency of $\lambda / 4$ short microstrip resonator.

\subsection{Manufacturing Issues of the Resonator Package}

The cross section in Figure 4-18, illustrates the components of the oscillator package. The hemispherical cavity resonator was embedded into the brass casing by using a flat headed drill bit of correct radius and depth to resemble a hemisphere. The downfall of this method is that the bottom center of the hemisphere has a small flat area caused by the drill bit. In the future, a custom milling tool could be made for drilling the hemispherical cavity. 
The PCB card made of Rogers Duroid 5880 is placed on the resonator and brass jig package but is not secured with epoxy. The use of conductive epoxy was ruled out in view of the fact that there was a high chance of it leaking into the hemisphere of the resonator and consequently altering its performance and resonant frequency. In addition, the epoxy has a much lower conductivity than the gold plated walls and would have therefore decreased the quality factor drastically. As an alternative, a gold plated clamp, securing the PCB to the brass casing with four screws, shown in Figure 4-19, was used in order to ensure that the duroid card was sitting flat onto the resonator with proper contact.

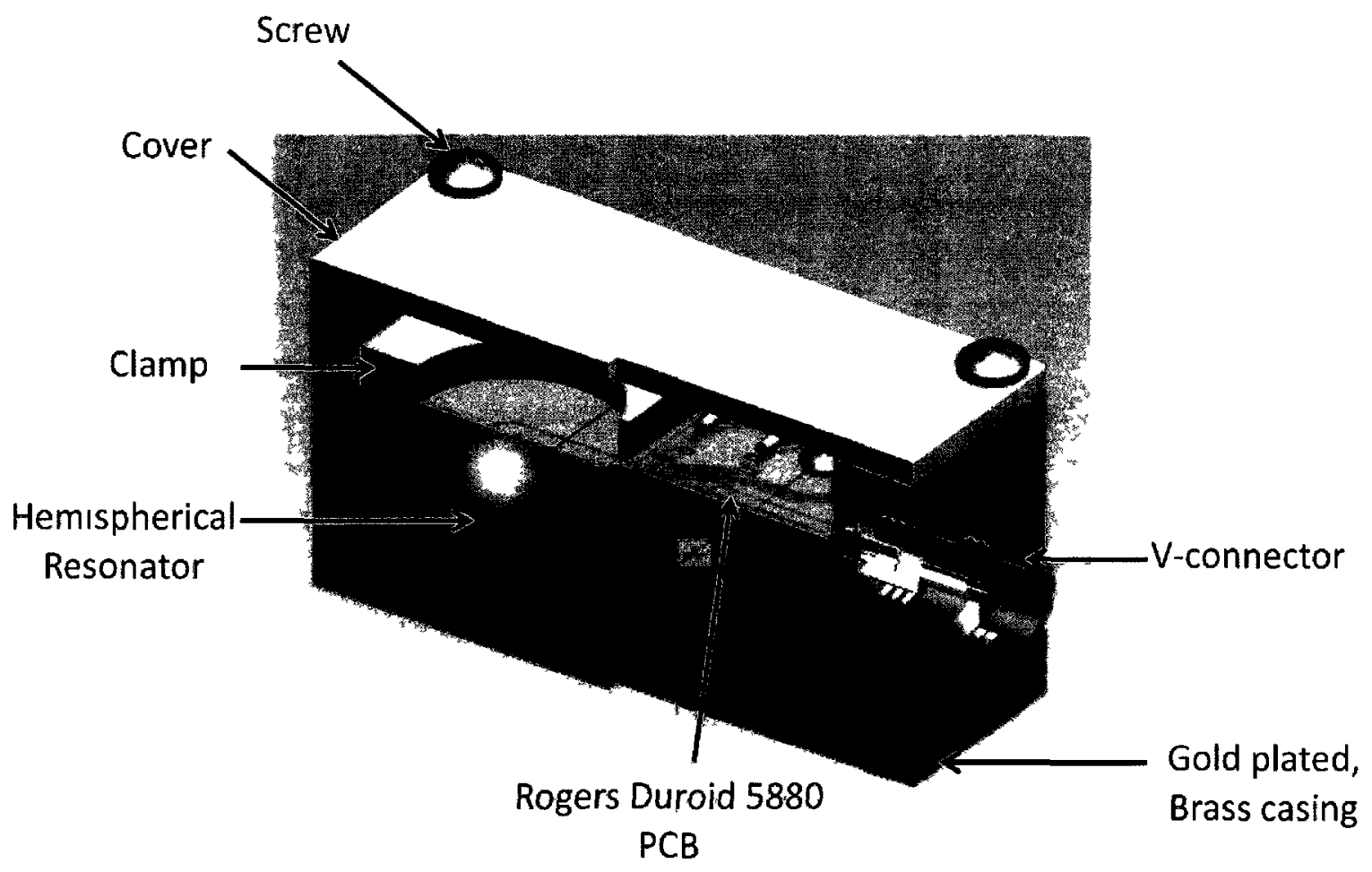

Figure 4-18: Cross section of oscillator package. 


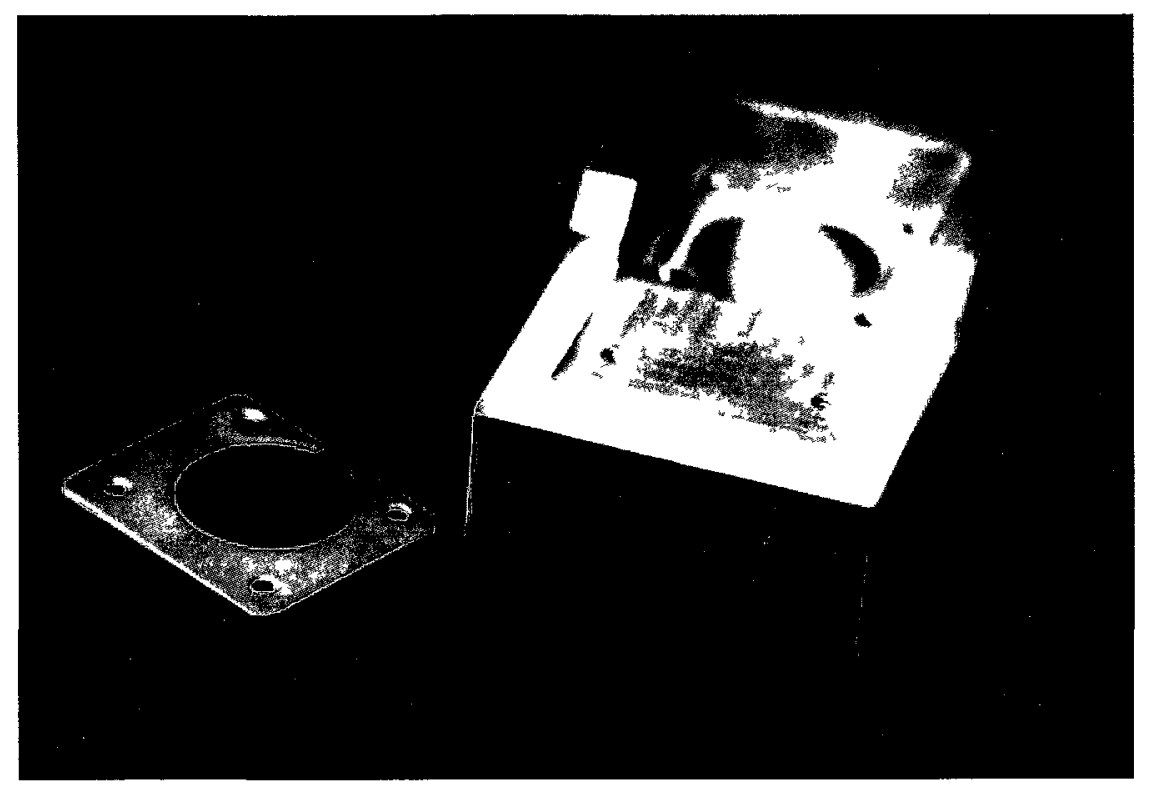

Figure 4-19: Gold plated clamp and resonator package

The gold-plated brass cavity was modified for resonator measurements as shown in Figure 4-20. The side walls on the other side of the hemispherical cavity were shaved off in order to allow the probes to come in safely onto the microstrip feed line without being damaged.

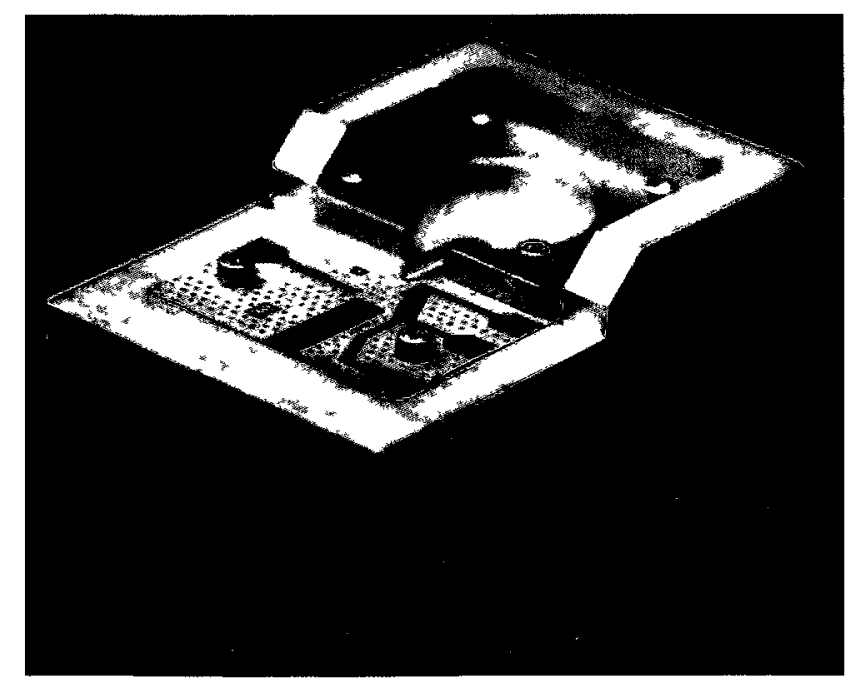

Figure 4-20: Modified package for probing of the resonator. 
The printed circuit board, from simulations and fabricated, designed by fellow student Han-ti Chuang, is shown in Figure 4-21 and Figure 4-22 respectively. Section A has been designed to accommodate the hemispherical cavity resonator and its loading mechanisms. Section B can be found in more detail in [7] with a complete description of each component in the oscillator. In section A, the hemispherical cavity resonator is embedded in the brass package and is therefore not visible from the top side of the duroid card. Similarly, the coupling aperture is located on the ground or bottom side of the duroid card and is also not noticeable from the top. This generated a big challenge when it came to the proper alignment of the coupling slot given that it has to be in the correct place in order to guarantee proper coupling and correct measurements. The problem was solved by using some of the components in section $\mathrm{B}$ as a reference system such as the Vconnector and the DC bias lines. The glass bead in the V-connector would be aligned perfectly with the launcher for measurements, ensuring good alignment in the horizontal plane, whereas the DC bias lines would be aligned with the filter con holes to ensure alignment in the vertical plane. This procedure allowed for the coupling aperture to be at the appropriate location for coupling to the microstrip feed and for the appropriate location with respect to the hemispherical cavity. 


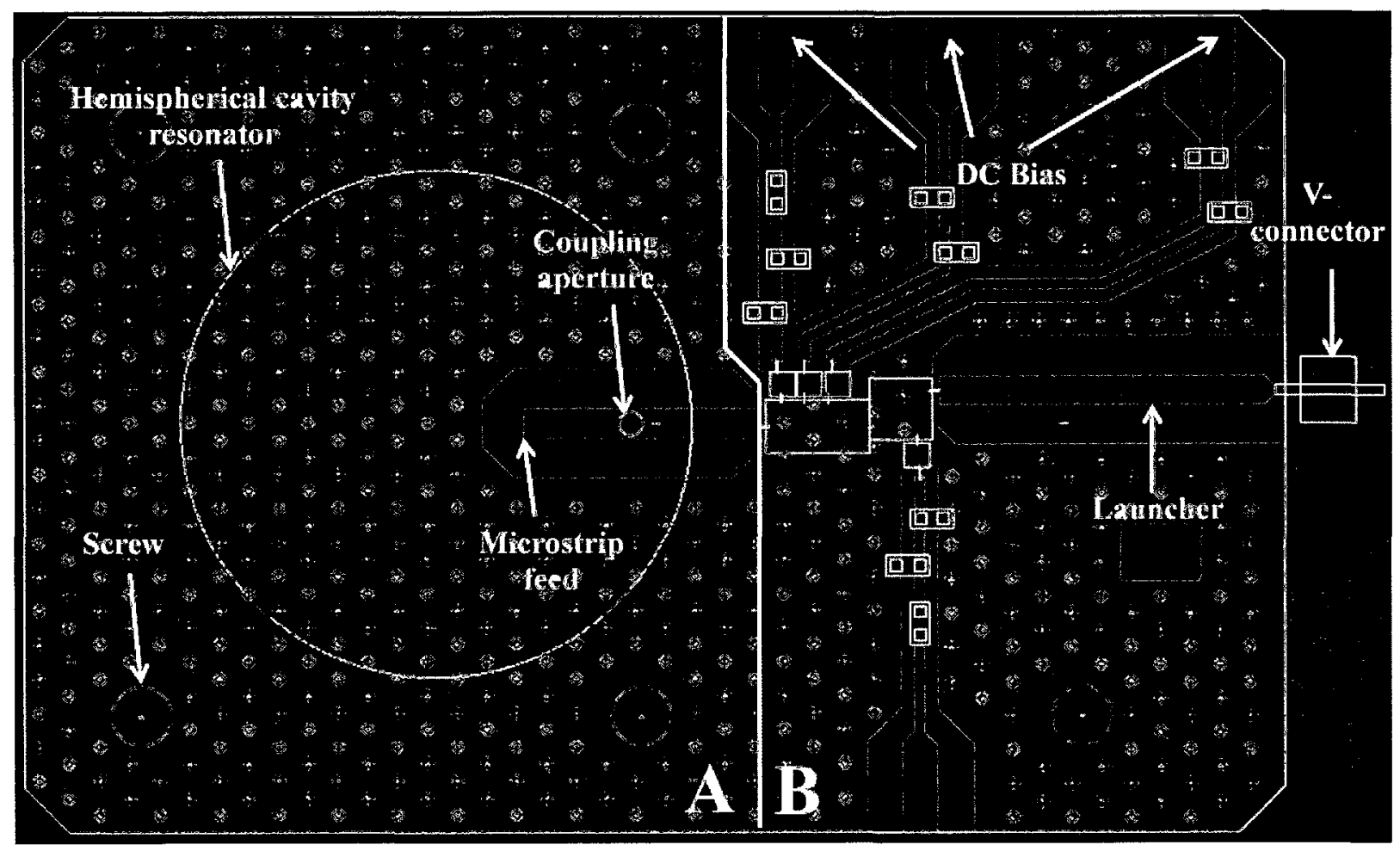

Figure 4-21: Floor plan of printed circuit board (from s1mulations)
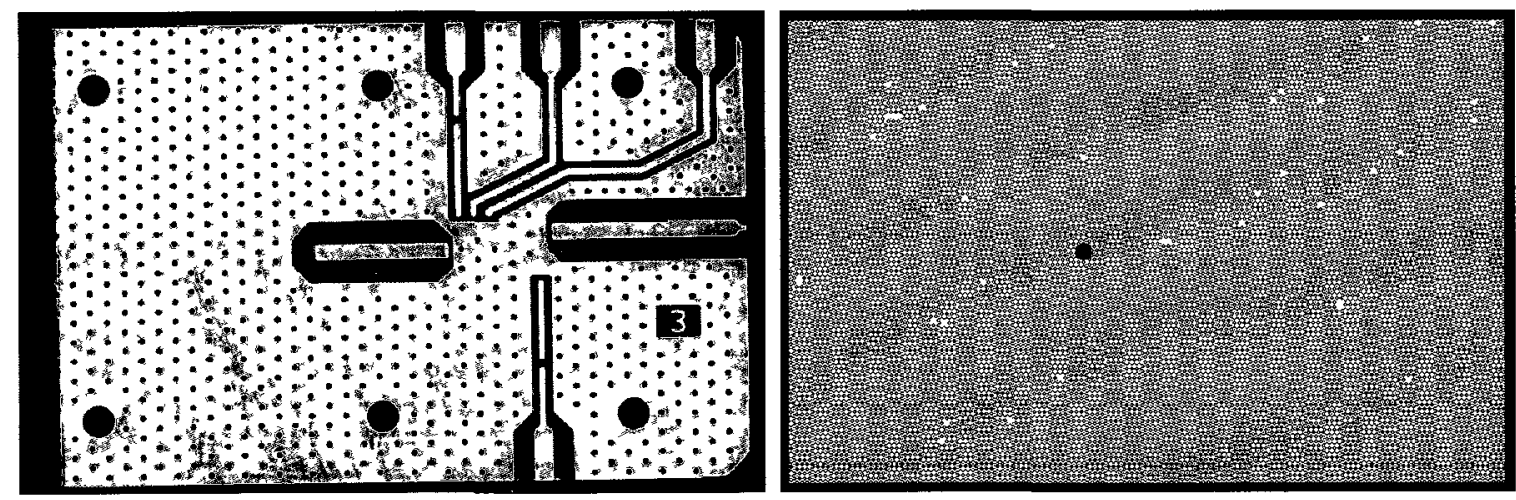

Figure 4-22: Floor plan of printed circuit board (fabricated),

top view and bottom view respectively. 


\subsection{A Tunable Hemispherical Cavity Resonator}

The hemispherical cavity resonator in Chapter 3 was designed at a frequency of 20 $\mathrm{GHz}$ to accommodate the oscillator for Han-ti Chuang's thesis. As previously stated in Eqns. (3.1-0) and (3.1-1), the frequency of the cavity resonator depends solely on the size of the cavity at a specific mode. This thesis also explores the possibility of realizing a hemispherical cavity resonator which can be tuned to a desired frequency.

Following manufacturing processes, the resonator cavity, as shown in Figure 4-23, will not be of exact dimension as calculated. This may change the frequency slightly and in this case, the tuning capability can become useful for a specific frequency required.

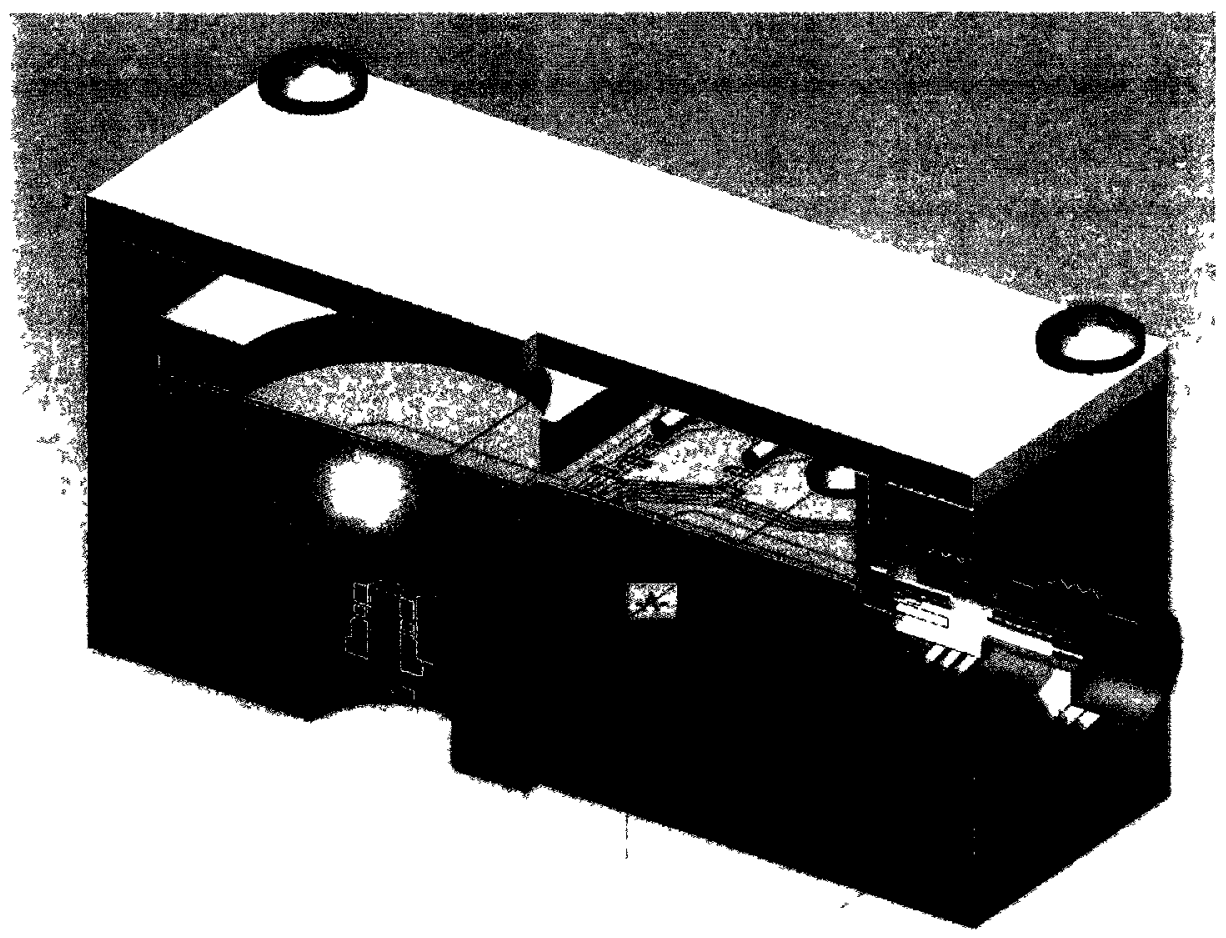

Figure 4-23: Tunable Oscillator Package 
The concept behind the tunability of the resonator was realized by inserting a gold plated screw in the bottom centre of the cavity, as shown in Figure 4-24, where the electric field is high and can be disturbed slightly.

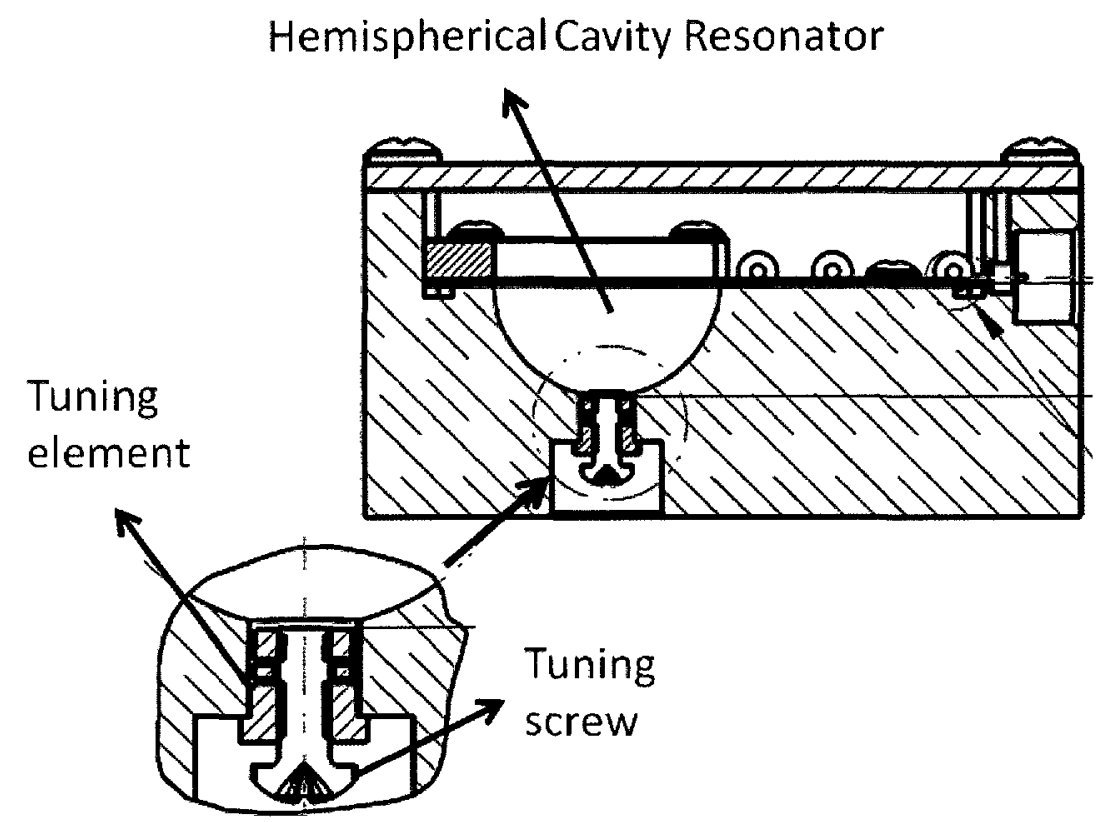

Figure 4-24: The concept behind the tunable cavity resonator.

The tuning element in Figure 4-24 is a Johanson posi-torque bushing with thread size $0.060 "$ " 44]. This tuning element was chosen for low loss and precision tuning as there is no need for locking nuts due to the self-locking, constant torque mechanism. A commercially available gold plated $0-80$ screw is also used for the precision tuning.

An analysis was done in HFSS to determine if the cavity resonator would be tunable. A cylinder made of a perfect electric conductor, mimicking the gold plated screw, is inserted in the centre of the bottom of the cavity, as shown in Figure 4-25. The depth of the screw into the cavity was varied in order to slightly disturb the electric field and determine if a change in frequency occurred. Figure 4-26 presents the change in frequency and loaded quality factor as the screw is inserted in the cavity resonator. 


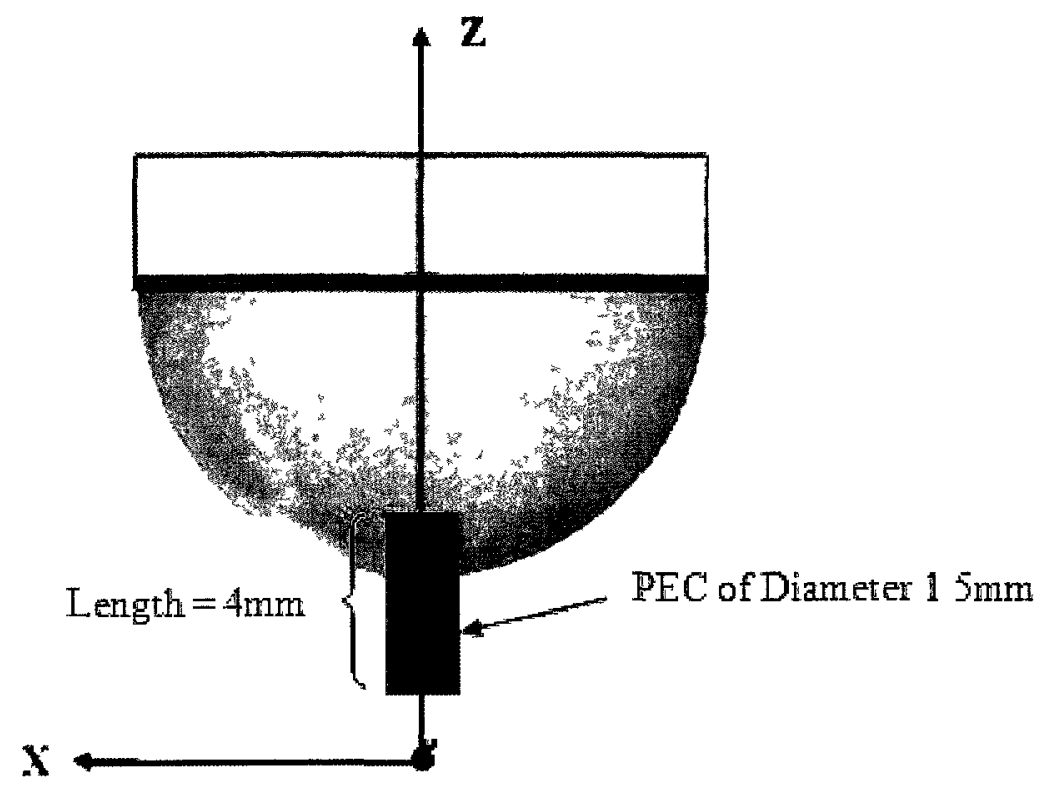

Figure 4-25: Tuning screw setup

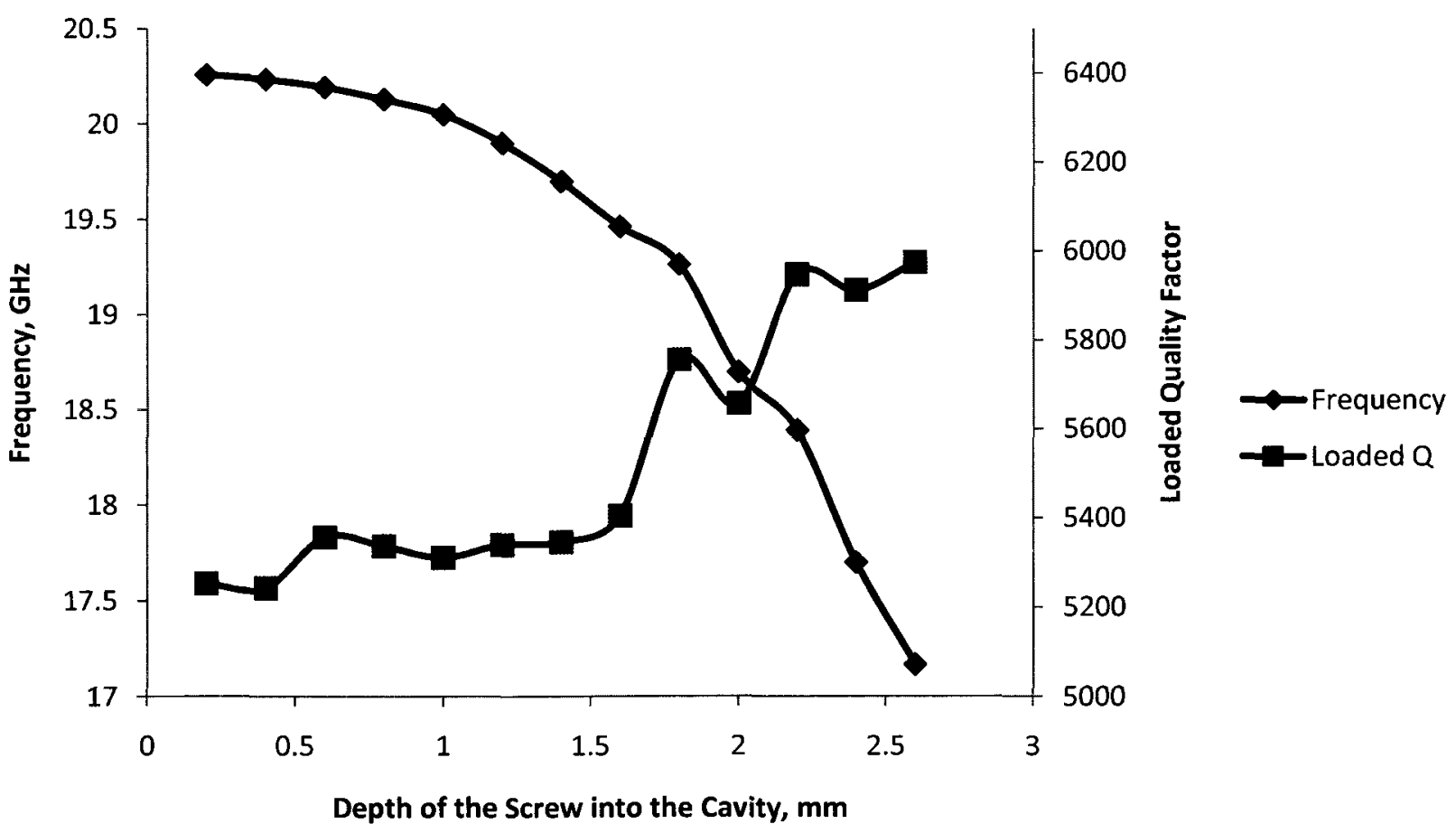

Figure 4-26: Depth of screw vs. Frequency vs. Loaded quality factor 
As can be seen from Figure 4-26, as the screw is inserted deeper into the bottom of the cavity, the resonant frequency decreases and the loaded quality factor increases slightly. This results in a tuning capability of about $3 \mathrm{GHz}$. 


\section{Chapter 5}

\section{Measurements and Performance of the Hemispherical Cavity Resonator}

This Chapter will present a comparison between measurement and expected simulated results using commercial EM software. Furthermore, the measurement setup used to characterize the hemispherical cavity resonator will be described. Finally, an analysis and discussion of some of the possible discrepancies between the measured and simulated results will be given.

\subsection{Resonator Fabrication and Dimensional Tolerances}

This section will introduce the actual resonator fabrication and will discuss some issues associated with dimensional tolerances in the Duroid card, in the hemispherical cavity resonator itself and will address issues that arose during the complete assembly. 


\subsubsection{RT/Duroid 5880 PCB layout issues}

The printed circuit board discussed in Section 4.4 was fabricated in RT/Duroid 5880 which in many respects is a non ideal material; it easily bends and can be subject to manufacturing tolerances such as over- and under-etching. The feature sizes on the PCB were measured under a microscope with high precision and can be found in Table 5-1 along with the values used during simulations. As can be seen, the radii of the measured aperture are larger than simulated. This is due to the under-etching during the manufacturing process. On the other hand, the width of the microstrip feed was found to be over-etched at the edges and is approximately 60-80 $\mu \mathrm{m}$ less than the simulated width.

Table 5-1: Simulated vs. Measured features on PCB

\begin{tabular}{|c|c|c|c|c|}
\hline \multicolumn{1}{|c|}{ PCB } & \multicolumn{2}{|c|}{ Aperture Radius, mm } & \multicolumn{2}{c|}{ Width of microstrip feed, $\boldsymbol{\mu m}$} \\
\cline { 2 - 5 } & Simulated & Measured & Simulated & Measured \\
\hline b3 & 0.300 & 0.318 & 763 & 688 \\
\hline b4 & 0.400 & 0.421 & 763 & 696 \\
\hline b5 & 0.500 & 0.522 & 763 & 683 \\
\hline b6 & 0.600 & 0.611 & 763 & 695 \\
\hline
\end{tabular}

Figure 5-1 presents the PCB card and the jig specifically designed to simplify resonator measurements. Gold plated vias were added in between conductors to secure proper ground plane for all components. CRC only has drill bits to create vias which are 10 mils, 12 mils and 13.5 mils in diameter and 12 mil drill bits were used in this project. An issue that was encountered in other versions of the PCB card was the plating of the vias. Some vias were not properly punched through and could be seen plugged to the naked eye when brought up to the light. This most probably was not a significant source of error due to the fact that there were so many vias providing the proper ground contact. 


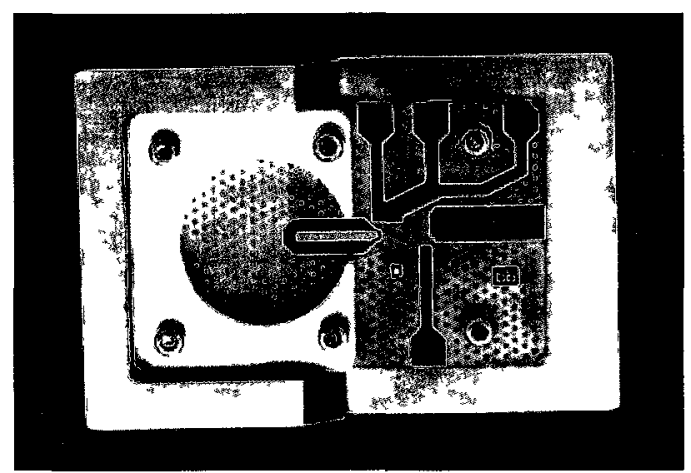

Figure 5-1: Resonator package and PCB complete assembly for measurements.

The de-embedding of the probe-to-pad transitions was also included in the measurements by tapering the microstrip feed on the duroid card. The coplanar GroundSignal-Ground probes were placed on the tapered feed as shown in Figure 5-2 and a probe-to-pad transition was replicated and measured for de-embedding. Figure 5-3 presents a zoomed picture of the probes.

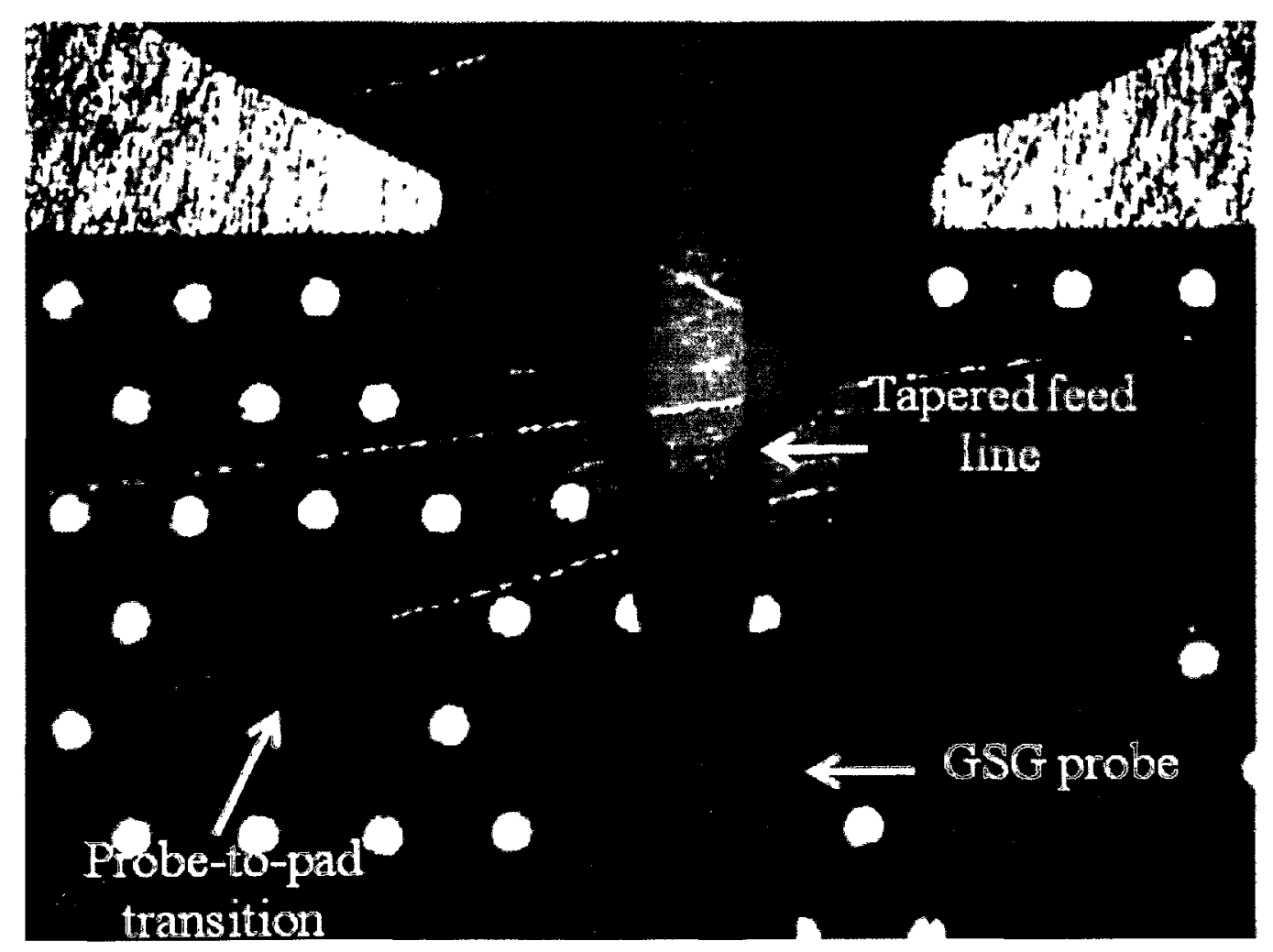

Figure 5-2: Actual layout of the probe-to-pad transition for de-embedding. 


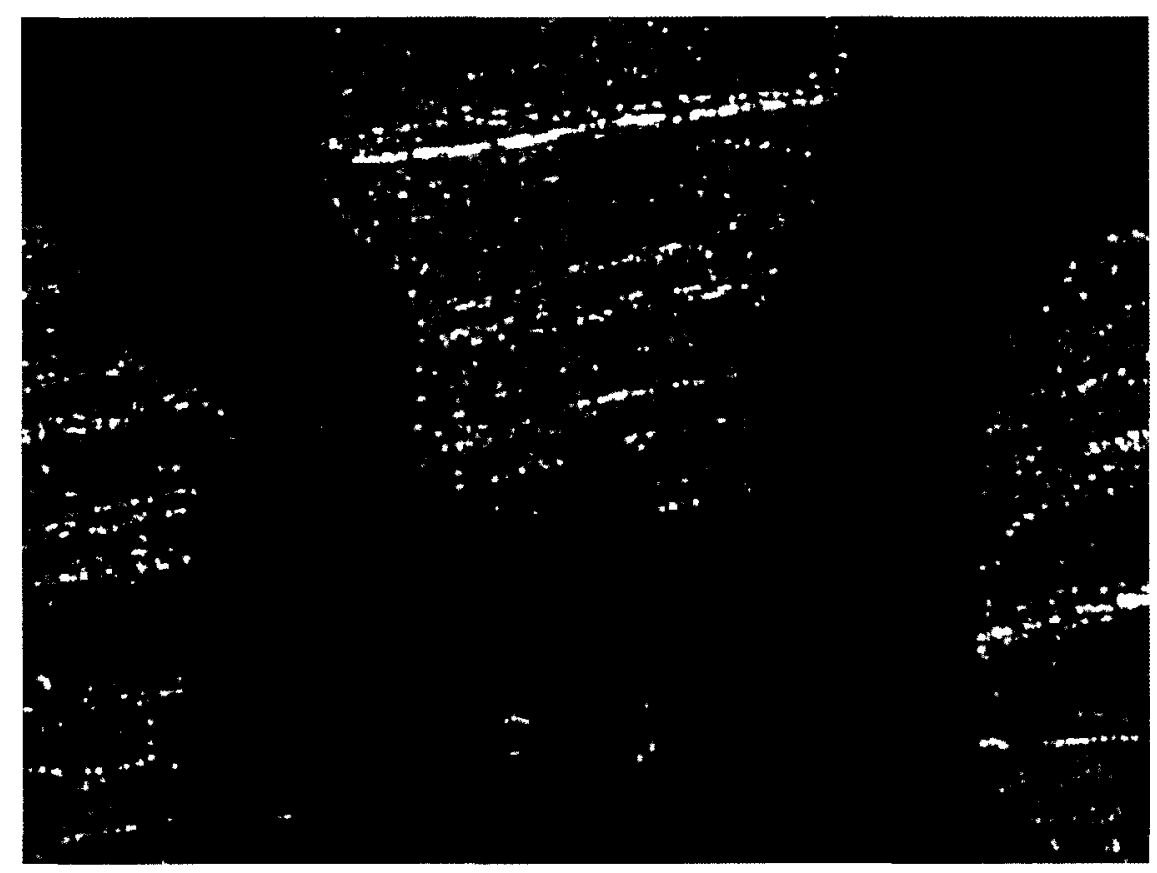

Figure 5-3: Zoom of the landing of the probes on the microstrip feed.

More specifically, the diagram in Figure 5-4 breaks up the steps taken to de-embed the pad. The complete setup along with equations used in ADS can be found in Appendix A. The $S_{11}$, meas data was collected by probing the tapered microstrip feed line as shown in Figure 5-2. The actual measurement needed is $S_{11}$, sim which is at a different reference plane that the actual measurement needed in order to compare the measured resonator with the simulated resonator. The probe-to-pad transition was replicated on the side of the board and measured as well. To de-embed the probe-to-pad transition with this setup and

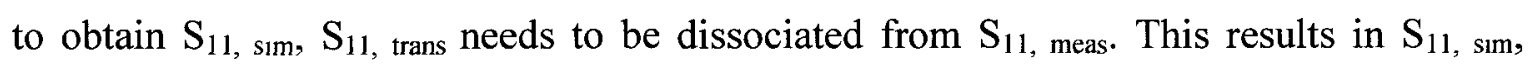
allowing the proper measurements to be compared as shown in Figure 5-4. 

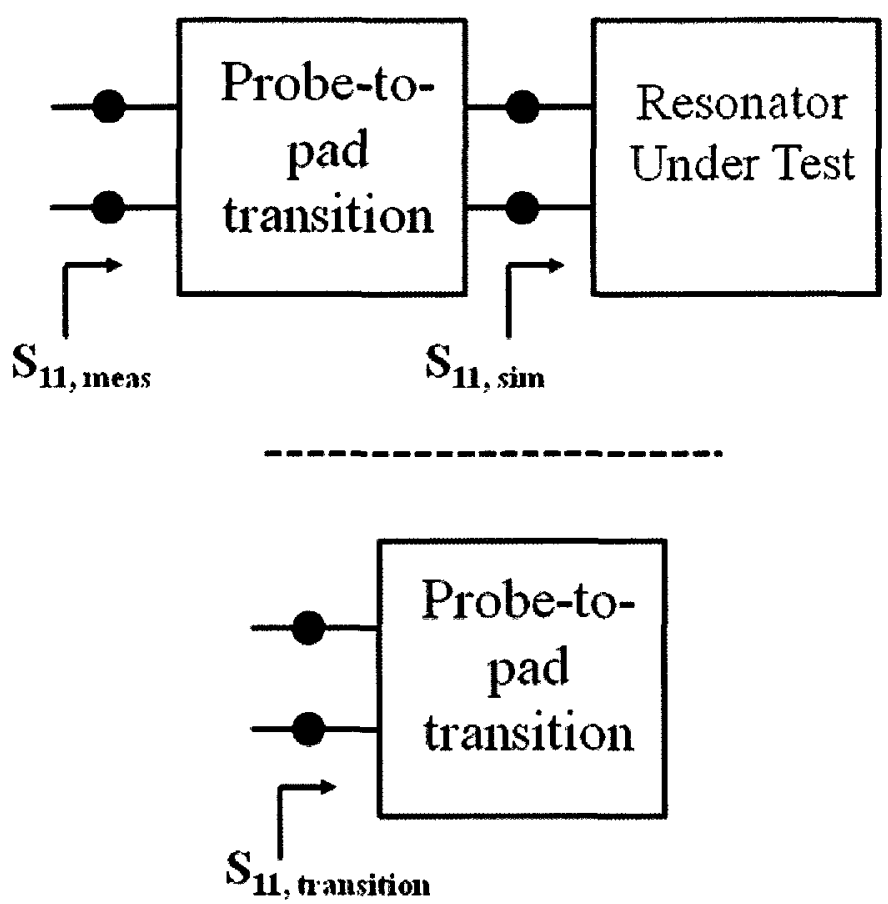

Figure 5-4: De-embedding the probe-to-pad transition

\subsubsection{Hemispherical Cavity machining}

The hemispherical cavity resonator embedded in the brass package was machined by using conventional machining techniques which are also subject to tolerances. The measured diameter of the fabricated hemispherical cavity resonator is $6.545 \mathrm{~mm}$ which is fairly accurate compared to the actual simulated diameter which is $6.54 \mathrm{~mm}$. It only presents about a $0.03 \%$ increase compared to the Rogers Duroid manufacturing errors with the aperture radius and width of the microstrip feed which had about a $50 \%$ increase and $9 \%$ decrease respectively. Other than this issue, the hemispherical cavity proved to be quite accurate as the same \% error was found in all jigs to be the same. This provides consistency if nothing else and proves that conventional machining is a safe way to go. 


\subsubsection{Complete Assembly}

The various components comprising the active circuitry of the oscillator were populated onto the PCB as per the layout which was in turn mounted onto the gold plated brass jig. The complete assembly can be seen in Figures 5-5, 5-6 and 5-7. The assembly of the package is critical for components such as the V-connector and the DC bias lines. Concerns such as alignment start coming into play. Although the latter are not directly related to the resonator, they serve as guidelines for some of the resonator parameters, as seen in Figure 5-6.

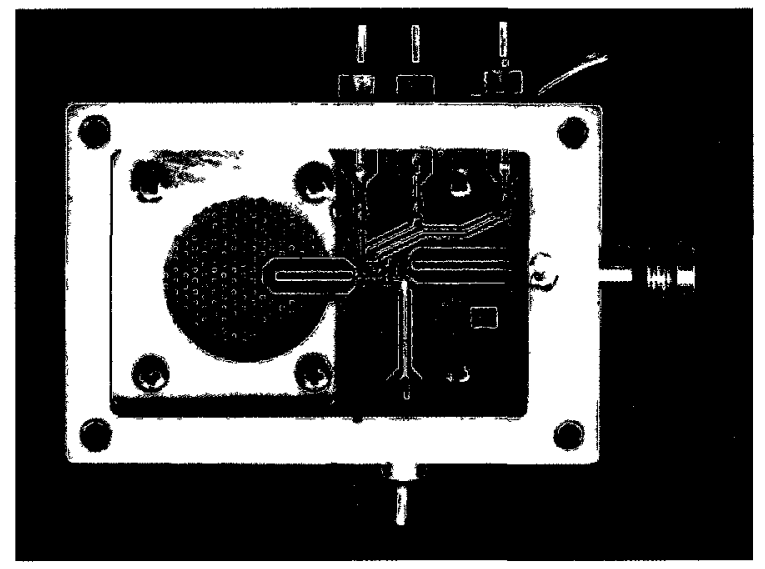

Figure 5-5: Top view of complete and assembled package.

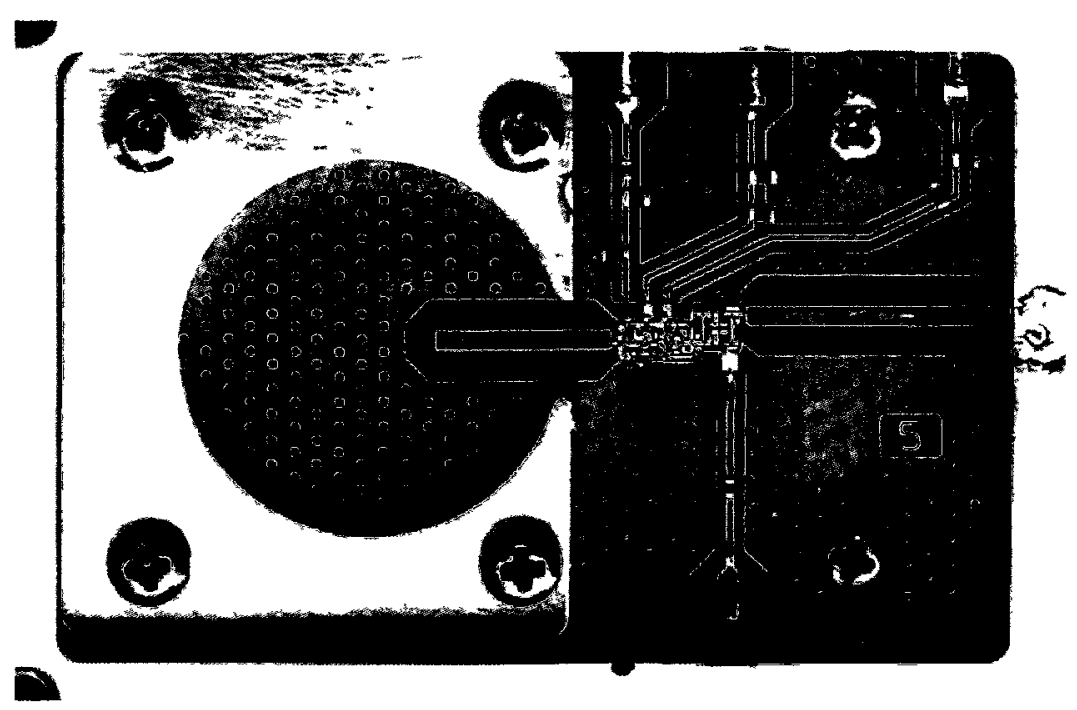

Figure 5-6: Populated PCB card for final assembly, detail in [7] 


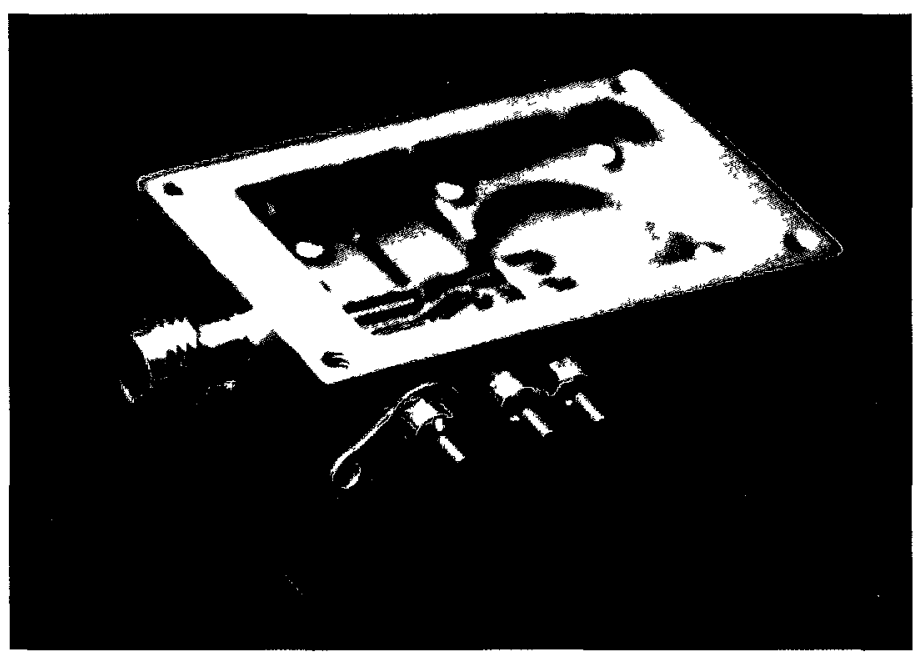

Figure 5-7: 3D view of complete and assembled package.

The aperture coupling slot located on the bottom ground plane of the substrate is not visible from the top of the card. This makes it difficult to align it properly $5 \mathrm{~mm}$ away from the centre of the hemispherical cavity even though the PCB was designed for it. When the PCB is being screwed into the jig, careful attention must be taken in order to keep the card as straight as possible. The alignment marks used for the complete PCB were also kept on the PCB for the resonator measurements only to ensure that the card was aligned correctly and to ensure that the coupling aperture was in fact at the location of maximum magnetic field.

\subsection{Equipment Setup}

The equipment used in the measurement setup for the hemispherical cavity resonator is shown in Figure 5-8 and consists of a prober from Karl-Süss and GSG probes with 500 $\mu m$ probe pitch. The gold plated brass jig was mounted on the chuck of the probing 
station and connected to an Agilent E8361C Network Analyzer via coaxial cables. This is in turn connected to a computer in order to save and analyze data.

Before measurements began, proper calibration was completed with an SHORTOPEN-LOAD-THROUGH configuration using the calibration kit from GGB industries SOLT_CS9_500 with Ground-Signal-Ground (GSG) configuration [45] in order to compensate and take into account the parasitic effects of some of the tools such as the cables and the probes themselves.

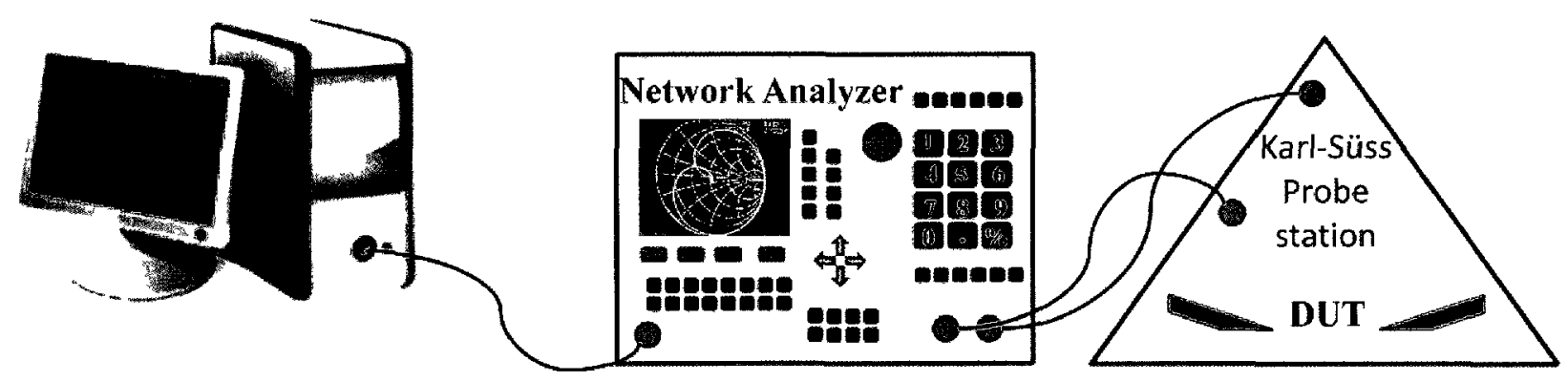

Figure 5-8: Measurement equipment and setup

The S-parameters obtained from the network analyzer were recorded and imported into QZERO [42] in order to extract the measured quality factors and compare them to the simulated S-parameter data already presented in Chapter 4.

\subsection{Performance and measurements of the hemispherical cavity resonator}

Section 3.6 presented a comparison between the calculated and the simulated loaded and unloaded quality factors of the hemispherical cavity resonator. This Section will present the results obtained using the equipment setup in Section 5.2 of the fabricated 
package embedded resonator. S-parameter narrowband measurement ( $19 \mathrm{GHz}-21 \mathrm{GHz})$ and wideband measurement $(10 \mathrm{GHz}-40 \mathrm{GHz})$ files were gathered and analyzed through the QZERO program. Narrowband files with 1601 points were used for the QZERO program to capture the resonant frequency and better estimate the quality factor. Figures 5-9 and 5-10 illustrate the measured S-parameters versus the simulated S-parameters for aperture radii of $0.5 \mathrm{~mm}$ and $0.6 \mathrm{~mm}$ respectively.

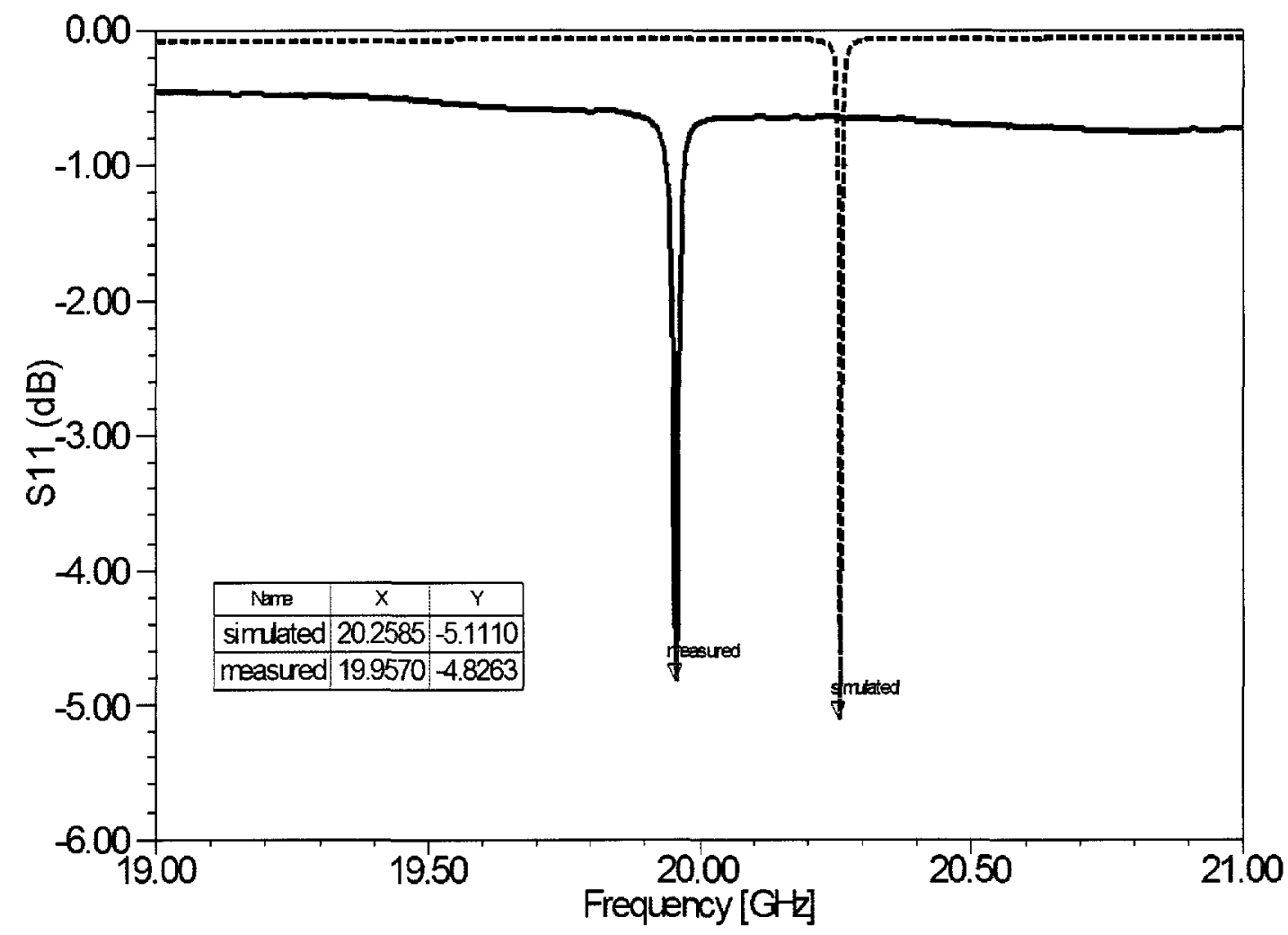

Figure 5-9: Measured $\mathrm{S}_{11}$ of cavity with coupling aperture $0.5 \mathrm{~mm}$ with corresponding simulated $\mathrm{S}_{11}$. 


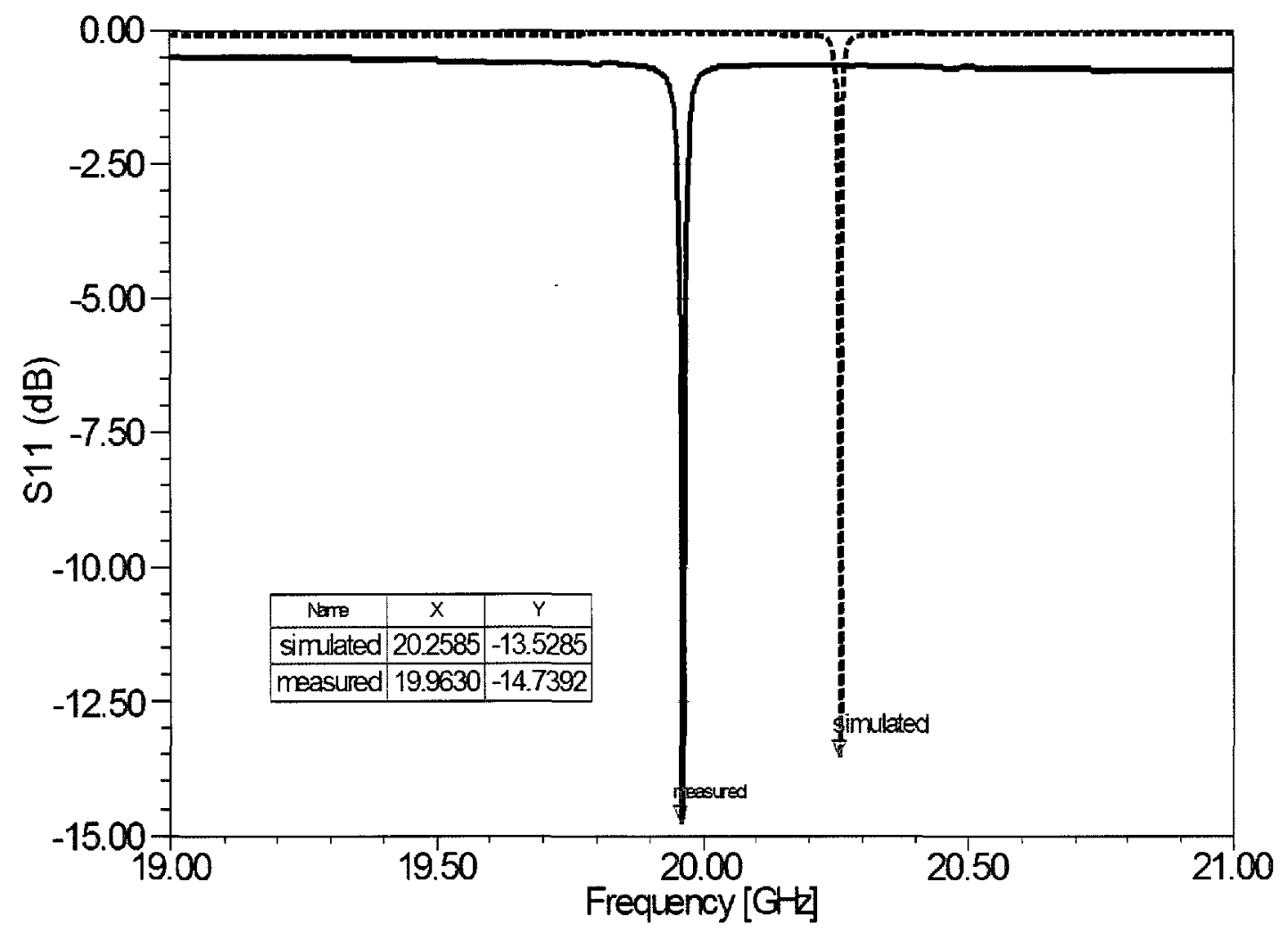

Figure 5-10: Measured $S_{11}$ of cavity resonator with coupling aperture $0.6 \mathrm{~mm}$ with corresponding simulated $\mathrm{S}_{11}$.

The Smith chart in Figure 5-11 provides a comparison between the measured and the simulated S-parameter data for aperture radii varying from $0.3 \mathrm{~mm}$ to $0.6 \mathrm{~mm}$. As can be seen, despite the resonator being designed at $20 \mathrm{GHz}$, the frequency of the measured cavity is slightly lower than the simulated frequency. The frequency change that can be seen see in Figures 5-9 and 5-10 can be explained by the fact that the actual measured cavity diameter is slightly bigger than the simulated diameter as was mentioned in Section 5.1.2. In addition, a revised simulation showed a similar down shift in frequency and hence explaining the phenomenon observed.

The simulated and measured quality factors are compared in Table 5-2 for different aperture radii. As expected, the measured unloaded quality factors are lower than predicted due to additional losses not fully captured in simulation. 


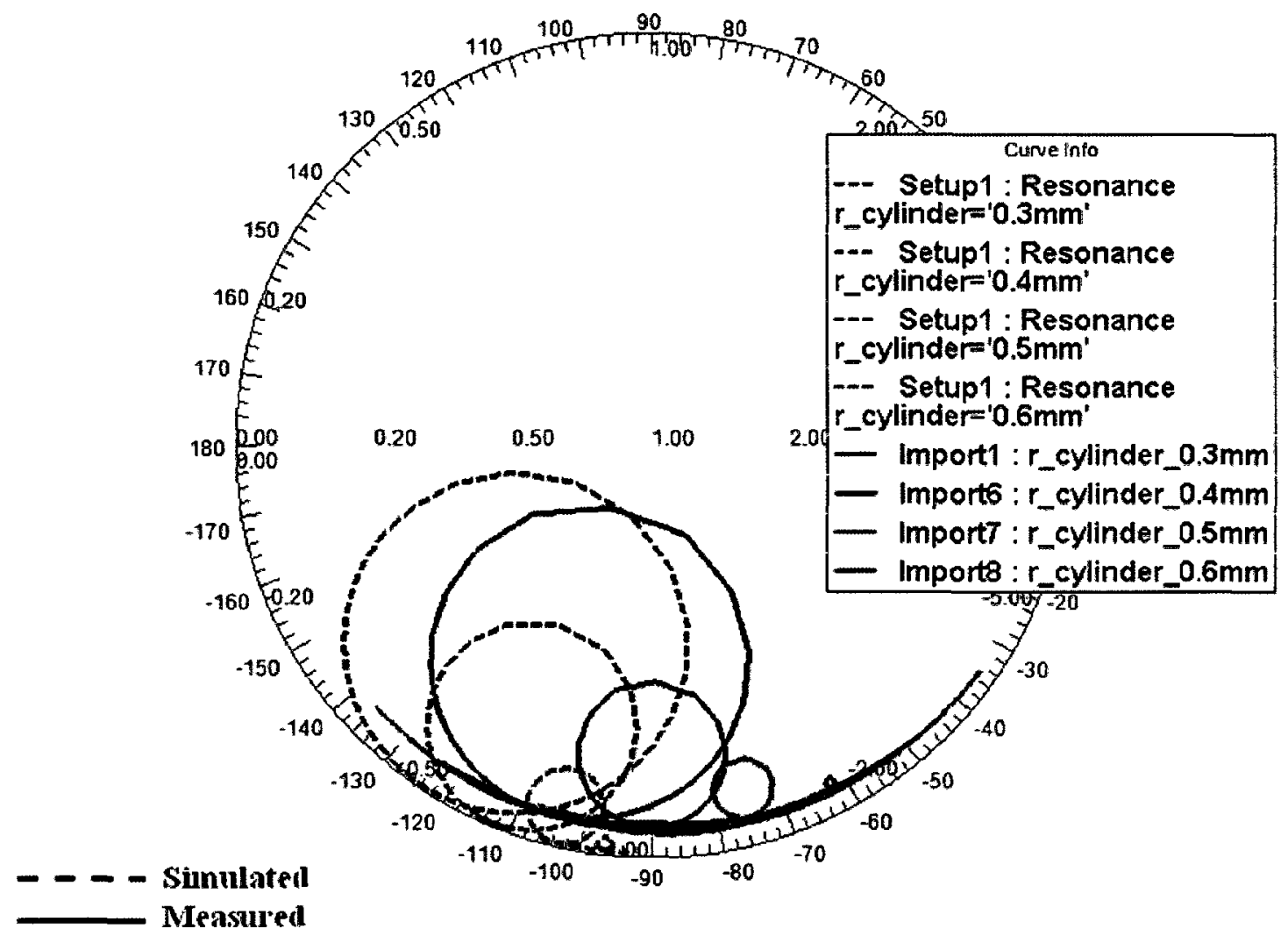

Figure 5-11: Measured versus simulated S-parameter data for various aperture radii.

Table 5-2: Simulated and measured quality factors for various aperture radii.

\begin{tabular}{|c|c|c|c|c|}
\hline \multirow{2}{*}{ PCB } & \multicolumn{2}{|c|}{ Unloaded Quality Factor } & \multicolumn{2}{|c|}{ Loaded Quality Factor } \\
\cline { 2 - 5 } & Simulated $^{*}$ & Measured $^{*}$ & Simulated & Measured \\
& & \\
\hline b3 & $5890 \pm 12$ & $2565 \pm 6.6$ & $5839 \pm 13$ & $2532 \pm 6.4$ \\
\hline b4 & $5402 \pm 9.2$ & $2298 \pm 14$ & $5399 \pm 7.1$ & $2125 \pm 13$ \\
\hline b5 & $5311 \pm 22$ & $2197 \pm 17$ & $4357 \pm 4.3$ & $1514 \pm 13$ \\
\hline b6 & $4800 \pm 19$ & $1855 \pm 25$ & $3086 \pm 5.6$ & $1318 \pm 14$ \\
\hline
\end{tabular}

*Note: error provided by QZERO. 
Following these results, an extra board was fabricated which included the effects of the ribbon wire used to connect to the VCO chip on the active side of the circuit. The measured S-parameters obtained from the network analyzer were inconclusive due to measurement issues. The gold plating on the substrate was very thin and did not allow for proper bonding of the wire, therefore connections were not properly established. Simulations however suggest that the slight change in frequency can be due to the ribbon bond.

The wideband spectrum was measured for a given aperture coupling radius of $0.6 \mathrm{~mm}$ and can be seen in Figure 5-12.

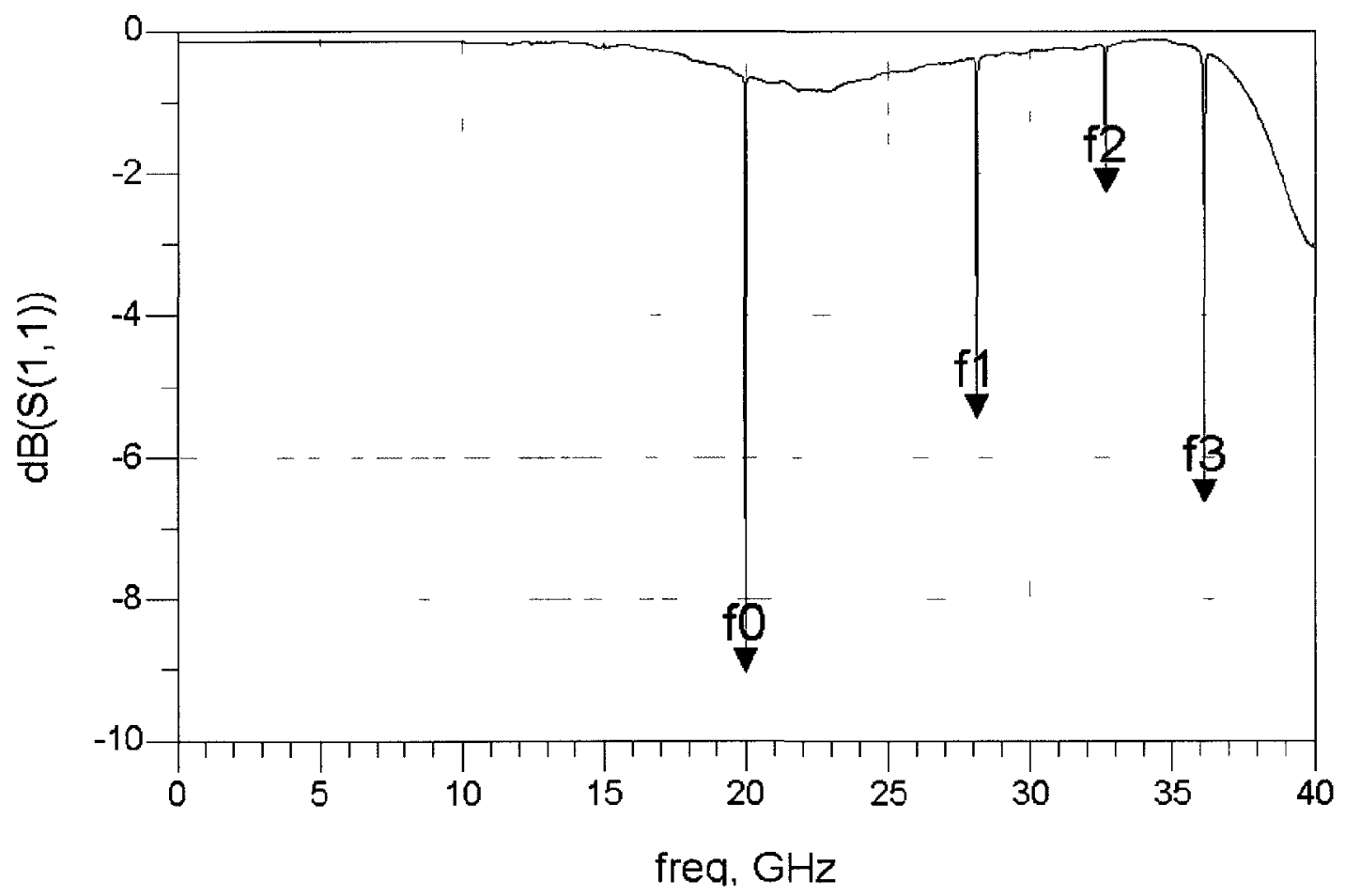

Figure 5-12: Measured wideband spectrum for an aperture radius of $0.6 \mathrm{~mm}$.

The centre resonant frequency $f_{0}$ is $19.96 \mathrm{GHz}$ with a return loss of $-9.03 \mathrm{~dB}, f_{l}$ is 28.12 $\mathrm{GHz}$ with a return loss of $-5.44 \mathrm{~dB}, f_{2}$ is $32.64 \mathrm{GHz}$ with a return loss of $-2.23 \mathrm{~dB}$ and finally $f_{3}$ is $36.13 \mathrm{GHz}$ with a return loss of $-6.64 \mathrm{~dB}$. Other than a slight shift in 
frequency in the measured wideband spectrum, it appears to be very comparable to the simulation results of the wideband spectrum obtained at the end of Chapter 3.

\subsection{Discussion of Results and Sources of Error}

The discrepancies between loaded and unloaded quality factor found in Table 5-2 can be caused by various sources which will be divided into categories and analyzed further in this section.

\subsubsection{Material Related}

One of the main sources of error is material related which can cause unexpected and additional losses. In simulations, the conductivity of gold was assumed pure $\left(4.1 \times 10^{7}\right.$ $\mathrm{S} / \mathrm{m}$ ) but in reality surface roughness and contamination may have decreased the conductivity and consequently increased losses. The thickness of the copper on the PCB may also have been reduced from $1 / 2 \mathrm{oz}$ to some lower amount contributing to the losses.

\subsubsection{Assembly and Design}

Another very probable source of error can come from the assembly of the package. Although a clamp was used to allow for the PCB to have a proper ground contact with the package, it may not have been applying uniform contact over the surface of the hemispherical cavity. This may have introduced a thin layer of air between the substrate conductor and the cavity itself. This was a hard task to simulate in HFSS. 


\subsubsection{Process Tolerances}

Process tolerances are also a great source of potential error. As seen in Table 5-1 the change in dimension of the microstrip feed results in a change of characteristic impedance and electrical length. Closely related to the dimensions is the fact that some of the boards had some contamination issues caused during fabrication. The microstrip feed often had some sputtering of copper close by which may have caused uneven lines and dimensions. Likewise, the coupling aperture occasionally had some dust-like particles of copper in it which had to be cleaned out manually and which may not have been cleaned fully.

A problem which occurred during the collection of the measured data was concerning the probes and probing machine. As mentioned in Section 5.2, before measurements began, a calibration kit was used to calibrate the machine in order to eliminate parasitic effects coming from the prober. After a couple of hours into the testing process, some of the S-parameter data became very noisy, presenting some irregularities. This was due to the fact that the probing machine began heating up and the calibration which was performed hours before was not longer valid. Recalibration was needed and although the problem was finally solved, some discrepancies may have occurred along the way for the other measurements. This problem was noted down and brought to the attention of the probing machine manufacturer.

Moreover, when the PCB boards were manufactured, the technical team advised us that a thin layer of gold was deposited onto the original substrate and onto the $1 / 2$ oz of copper already present. This was not accounted for in simulations and could have caused some additional losses.

\subsubsection{Simulation Assumptions}

In addition to the above sources mentioned, a further critical factor is the various assumptions made during simulations. The skin depth at a frequency of $20 \mathrm{GHz}$ is only approximately $0.55 \mu \mathrm{m}$ for gold. This suggests that even if the surface roughness is very little, the loss created is not negligible. The surface roughness in simulations was 
assumed to be zero, signifying that this could potentially be one of the major areas where losses were neglected when they should not have been. At low frequencies, the depth of the current penetration (and hence the electromagnetic fields), will usually exceed the surface roughness, and therefore the surface roughness will have no effect. At high frequencies $>1 \mathrm{GHz}$ however, the fields will concentrate on the outside of the conductor, or in a very thin layer inside. Although the brass package had about $3 \mu \mathrm{m}$ of gold, the surface roughness was not taken into account, hence the high loaded quality factor in simulations. Similarly, as discussed in Section 3.3, the surface resistance $R$, was taken into account, but is not ideal and in reality lies between $R$ and $2 R$. Ideal surface resistance assumes no roughness, a perfect shape and perfect alignment [8] making it very difficult to determine which factor is creating the greatest loss.

\subsection{E-band Oscillator Phase noise}

The hemispherical cavity resonator designed in this work was intended to be used as a high quality factor resonator for application in an E-band Oscillator [7]. As discussed in Chapter 1 and 2, the phase noise of a microwave oscillator can be decreased by utilizing a high quality factor resonator as shown in Leeson's equation in Eqn (5.5-1):

$$
L=10 \log \left\{\frac{G F K T}{2 P}\left[\left(\frac{f_{0}}{2 Q}\right)^{2}\left(\frac{f_{\alpha}}{f^{3}}\right)+\left(\frac{f_{0}}{2 Q}\right)^{2}\left(\frac{1}{f^{2}}\right)+\frac{f_{\alpha}}{f}+1\right]\right\}
$$

As can be seen, the phase noise is inversely proportional to the square of the quality factor; therefore a resonator with high quality factor will present a lower phase noise than that of a lower quality factor resonator.

The system specifications for the E-band oscillator are revisited in Table 5-3. 
Table 5-3: Review of System Specifications

\begin{tabular}{|c|c|}
\hline Parameter & Required Specification \\
\hline Frequency & $80 \mathrm{GHz}$ \\
\hline Modulation Type & 256 QAM \\
\hline Data Rate & $1.5 \mathrm{Gbps}$ \\
\hline Oscillator Phase noise & -106 dBc/Hz@100 KHz \\
\hline Resonator loaded Q & $>2000$ \\
\hline
\end{tabular}

Although the high quality factor resonator did not present the quality factor expected in simulations, measurement results still show a loaded quality factor greater than 2000 which is sufficient to meet the system specifications of the oscillator. Increasing the quality factor beyond 2000 would not drastically affect the performance of the oscillator since simulations were performed and proved that having a resonator with a quality factor greater than 2000 would provide virtually no improvement of the phase noise [7]. Figure 5-13 provides a plot of the phase noise captured by the Signal Source Analyzer (SSA) for the E-band oscillator [7]. 


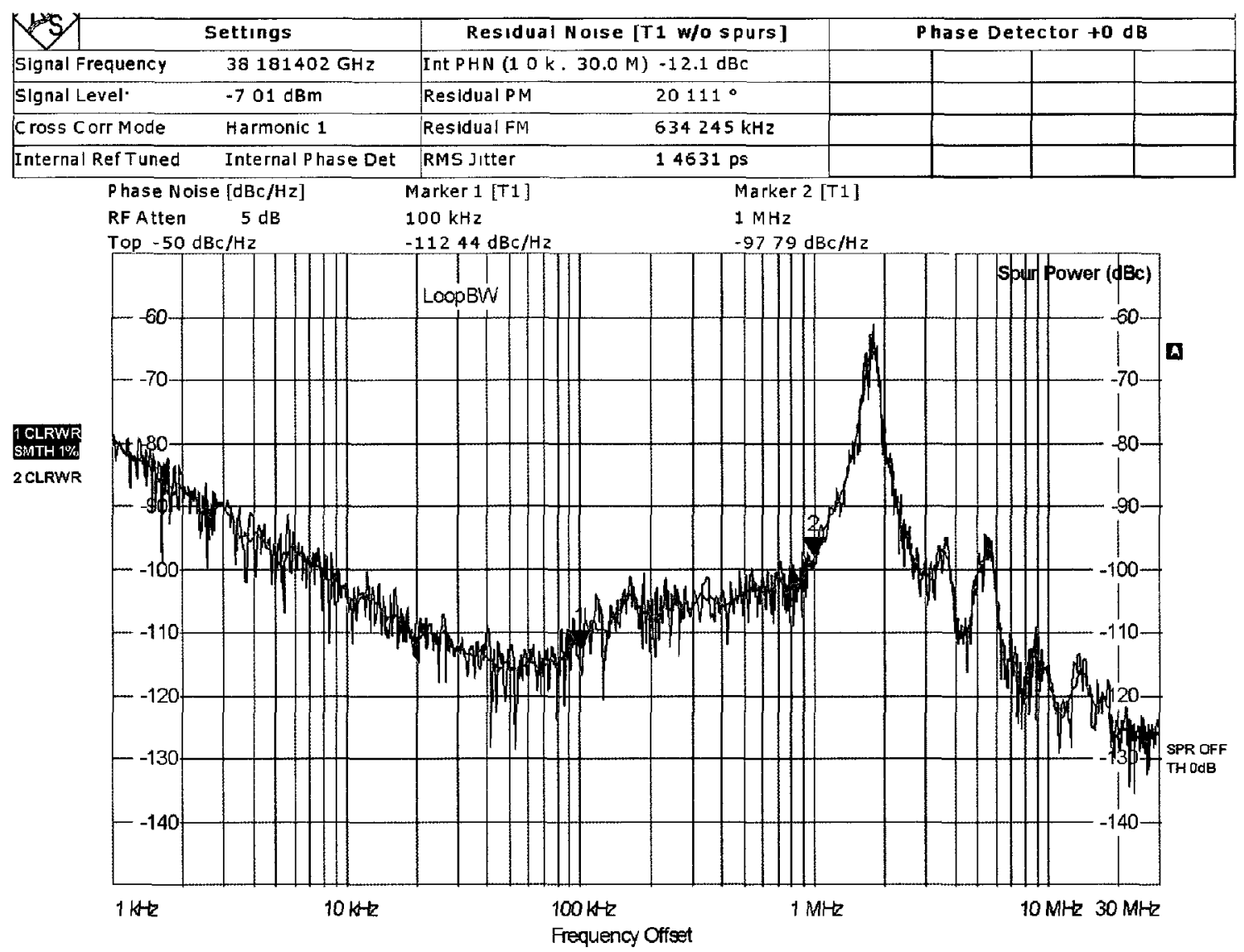

Figure 5-13: Phase noise of the E-band oscillator

The resonator, at $20 \mathrm{GHz}$, was ribbon bonded to the active signal source with an internal multiplier which brought the output frequency up to $40 \mathrm{GHz}$. The signal frequency in Figure $5-13$ is approximately $38.18 \mathrm{GHz}$, which is close to the $40 \mathrm{GHz}$ expected at the output of the signal source. The phase noise obtained at the frequency offset of $100 \mathrm{KHz}$ is $-112.44 \mathrm{dBc} / \mathrm{Hz}$ which is very close compared to the phase noise expected at $40 \mathrm{GHz}$ which is $-112 \mathrm{dBc} / \mathrm{Hz}$. The phase noise at $80 \mathrm{GHz}$ is expected to be $-106 \mathrm{dBc} / \mathrm{Hz}$ and therefore due to the $-6 \mathrm{~dB}$ degradation it results in $-112 \mathrm{dBc} / \mathrm{Hz}$ at $40 \mathrm{GHz}$.

The irregularities and the odd behavior that can be seen at frequency offsets greater than $100 \mathrm{KHz}$ in Figure 5-13 are due to tracking issues of the measurement device and have been present in other projects as well. It is a problem that needs to be solved by the manufacturer of the device and has been signaled. Also, it was quite difficult to lock the carrier frequency in order to obtain a steady phase noise plot which explains the fact that 
half the plot shows a representative phase noise measurement while the second half of the plot (beyond $100 \mathrm{kHz}$ offset) shows a variable signal.

The phase noise plot can however be extrapolated as shown in Figure 5-14. At a frequency offset of $100 \mathrm{kHz}$ the extrapolated phase noise is even better than previously stated and reaches approximately $-120 \mathrm{dBc} / \mathrm{Hz}$.

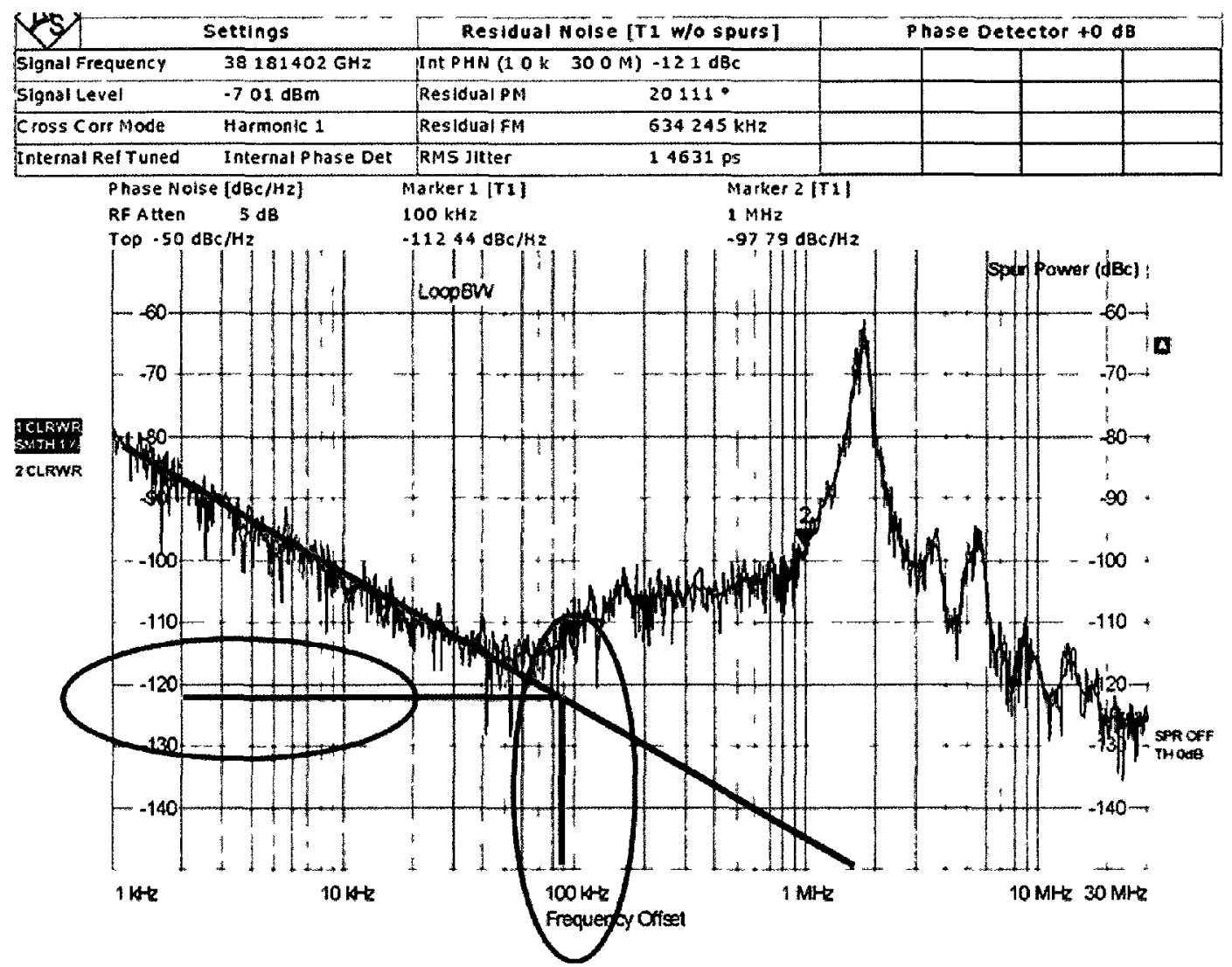

Figure 5-14: Extrapolated phase noise at $100 \mathrm{kHz}$ offset.

\subsection{Manufacturing issues with the tuning capability of the resonator}

The tuning capability of the resonator as described in Chapter 4 has been implemented and simulated. A tunable version of the package embedded resonator has been fabricated 
but due to some manufacturing issues with the drilled hole in the centre of the cavity, measurements have been delayed.

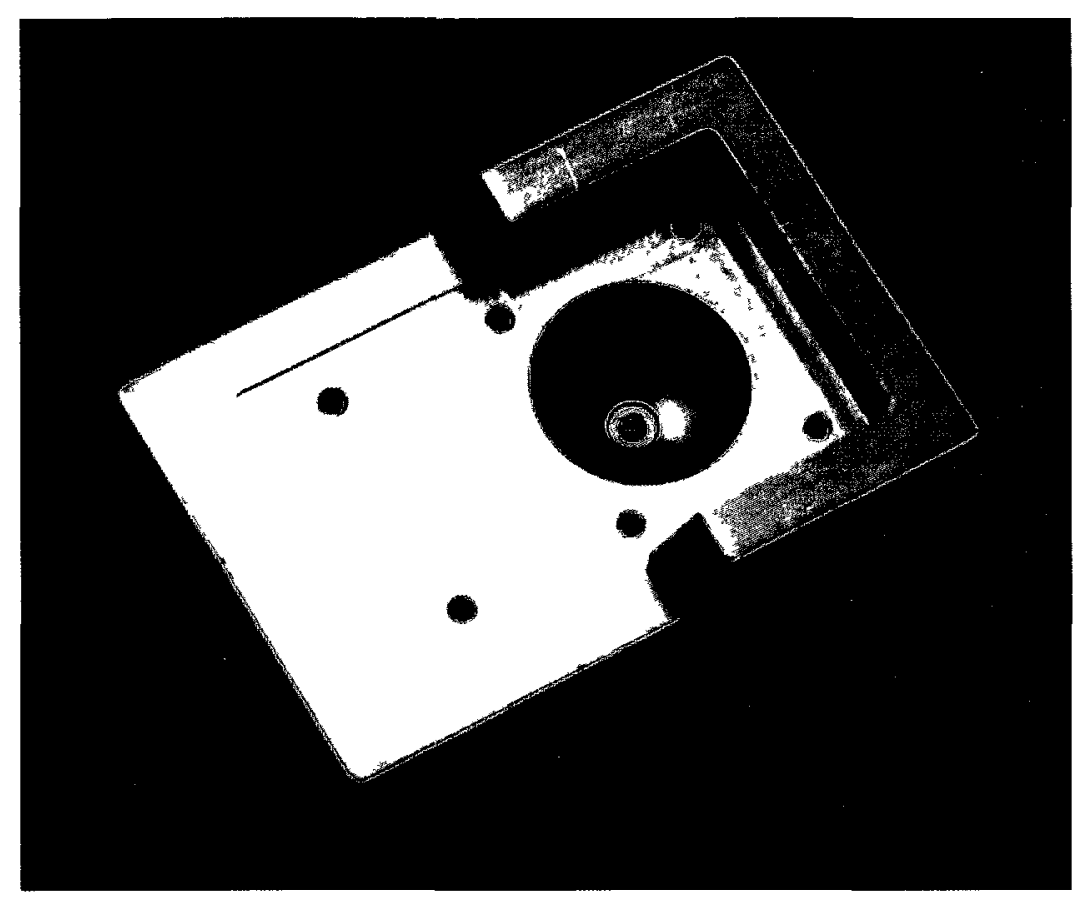

Figure 5-15: Manufacturing issue of the tunable resonator.

Figure 5-15 provides a 3D view of the resonator. As can be seen, the gold plated positorque element for the screw is currently not sitting tangential to the drilled hole. This is causing a gap of air whereas it should be completely filled up and the only thing moving back and forth should be the screw. In fact the screw is not penetrating the hemispherical cavity enough for tuning. This is issue is most likely due to the drilled hole being too small for the posi-torque element to fit properly. To fix this issue, the drilled hole will be slightly enlarged. 


\section{Chapter 6}

\section{Conclusions and Future Work}

The main goal of this thesis has been achieved in spite of the fact that the hemispherical cavity resonator described in this project proved to have some weaknesses in the design. The objective was to design a high quality factor resonator at a resonant frequency of $20 \mathrm{GHz}$. Although the loaded and unloaded quality factors are not as promising as the simulated results, a loaded quality factor of 2532 and an unloaded quality factor of 2565 were achieved at a resonant frequency of $19.96 \mathrm{GHz}$.

The hemispherical cavity resonator was successfully embedded in the gold plated brass package and successfully fabricated.

This final Chapter summarizes the results obtained, briefly retraces the steps which were involved from the design process to the measured results and highlights some research ideas for future projects.

\subsection{Summary}

The study and research of wireless applications and communications at high frequencies is a continuous and on-going process which is becoming more promising as the years go by. Creating these applications at E-band frequencies in a quick and low cost fashion is what more and more industries are striving for nowadays. The E-band channels are allowing users to transmit greater data rates while still using simple architecture, making it a very attractive band to study. 
This thesis expanded on the application of a high quality factor hemispherical cavity resonator which was modified to be embedded in a gold plated brass cavity for application in a low phase noise E-band oscillator.

Chapter 2 provided an overview of basic resonator theory along with a literature review of some typical resonators with relative pros and cons. Chapter 3 presented the theoretical design concept and initialized unloaded simulations with the electronic simulator Ansoft HFSS.

Chapter 4 comprised of an in depth analysis of the parameters which might have affected the quality factor of the resonator in order to ensure a loaded quality factor greater than 2000 to accommodate the E-band oscillator. The low phase noise of the oscillator is highly dependent of the resonator designed in this work.

The initial design was inspired by S. R. McLelland's $\mathrm{PhD}$ thesis on micromachined hemispheroidal cavity resonators [8]. Although a different approach was taken for the actual machining of the cavities and for the package which was needed to accommodate the E-band oscillator as well.

The main focus of this research project was centered around a low-cost and high quality factor resonator. These two objectives were brought to life by creating a brass package to house the circuit. Brass is relatively inexpensive and although it was sent out to Montreal for $3 \mu \mathrm{m}$ of gold plating, it has proved to be easy to work with at the Communication Research Centre (CRC). The hemispherical cavity is simple to reproduce in large quantities thanks to the accuracy of the machining process. Once the tool is designed for the shape required, reproduction accuracy does not become an issue. Furthermore, an abundant economical millimeter wave material, RT/Duroid 5880, was used to facilitate testing thanks to its quick return time for fabrication and attractive and high performance properties such as a low loss tangent of 0.0009. This allowed for various versions of the $\mathrm{PCB}$ to be made and allowed more room for adjusting last minute details.

Multiple PCB cards were fabricated in order to compare the different sizes of aperture radii with corresponding loaded and unloaded quality factors. A summary of the measured results is as shown: 
Table 6-1: Summary of measured results.

\begin{tabular}{|c|c|c|c|}
\hline PCB & Aperture Radius, $\mathbf{m m}$ & Unloaded Q & Loaded Q \\
\hline b3 & 0.318 & $2565 \pm 6.6$ & $2532 \pm 6.4$ \\
\hline b4 & 0.421 & $2298 \pm 14$ & $2125 \pm 13$ \\
\hline b5 & 0.522 & $2197 \pm 17$ & $1514 \pm 13$ \\
\hline b6 & 0.611 & $1855 \pm 25$ & $1318 \pm 14$ \\
\hline
\end{tabular}

Similarly, a summary of the simulated results is as follows:

Table 6-2: Summary of simulated results.

\begin{tabular}{|c|c|c|c|}
\hline PCB & Aperture Radius, mm & Unloaded Q & Loaded Q \\
\hline b3 & 0.300 & $5890 \pm 12$ & $5839 \pm 13$ \\
\hline b4 & 0.400 & $5402 \pm 9.2$ & $5399 \pm 7.1$ \\
\hline b5 & 0.500 & $5311 \pm 22$ & $4357 \pm 4.3$ \\
\hline b6 & 0.600 & $4800 \pm 19$ & $3086 \pm 5.6$ \\
\hline
\end{tabular}

The measured and simulated results exhibit some divergence from one another. The measured loaded quality factor differs in the range of approximately $\sim 1700-3300$ compared to the simulated loaded quality factor whereas the unloaded quality factor differs of about $\sim 2900-3300$ compared to the simulated unloaded quality factor. As discussed in Section 5.4 this large discrepancy is most likely due to the fact that too many assumptions were made in the simulation environment when it came to the losses of the board and of the cavity resonator itself. Although the $3 \mu \mathrm{m}$ of gold plating was taken into account, surface resistance and surface roughness were assumed ideal. This is obviously 
not the case especially at frequencies in the $\mathrm{GHz}$ range and can easily explain the origin of the losses in the circuit and hence the difference in the loaded and unloaded quality factors.

\subsection{Contributions}

The work outlined in this thesis contributed a novel package embedded hemispherical cavity resonator with a high loaded quality factor.

The primary contributions are listed in the following points:

- A hemispherical cavity resonator was effectively designed at a frequency of 20 $\mathrm{GHz}$ with the use of the 3D FEM simulator HFSS. The simulation resonant frequency was determined to be $20.26 \mathrm{GHz}$ and a maximum loaded quality factor of 4800 was achieved with a small aperture coupling slot.

- An in depth sensitivity analysis was performed on the parameters most likely to affect the loaded and unloaded quality factors of the resonator in order for future debugging to be simpler and more logical.

- The hemispherical cavity resonator embedded in the gold plated brass package was successfully built using commercial machining techniques and gold plated $3 \mu \mathrm{m}$ of gold.

- Testing of the cavity resonator was also completed, yielding a resonant frequency of $\sim 19.96 \mathrm{GHz}$ for all boards tested. The highest loaded and unloaded quality factors were obtained for the smallest aperture coupling slot and were 2565 and 2532, respectively, providing required specifications for the application of the E-band oscillator [7]. 


\subsection{Future Work}

This final Section reflects on the potential studies which could be done to help strengthen the work that has been presented in this thesis as well as to come up with ideas for future applications in this field.

To improve and strengthen the design of this thesis two things can be done:

- In order to improve the work discussed in this thesis one should attempt to understand the origins of the losses in the design. Some possibilities have been discussed and should be taken into account in the next iteration of the design. Although it has been concluded that many losses are difficult to accurately quantify, too many assumptions were made and the design was assumed mostly ideal. The next step would be to calculate and add some more realistic assumptions for the resonator and material losses.

- The material used in the PCB card can be replaced by a more durable and dependable material such as alumina. Duroid created some problems during the fabrication which can be avoided by using a material that does not bend or etch as easily.

Future efforts and projects could include:

- Creating the same hemispherical cavity resonator but adding several in parallel. This would increase the loaded quality factor further and could be promising especially at higher frequencies. At higher frequencies, the cavity is smaller in size and therefore adding a couple in parallel would not take up an excessive amount of room. 
- Furthermore, the concept of tunability can be further explored by using dielectric screws instead of commercially available gold plated screws. Dieletric tuning screws would allow for a finer more accurate tuning which would be useful for precision tuning. 


\section{Appendix A}

\section{De-embedding Setup for resonator measurements}

From Advanced Design System:

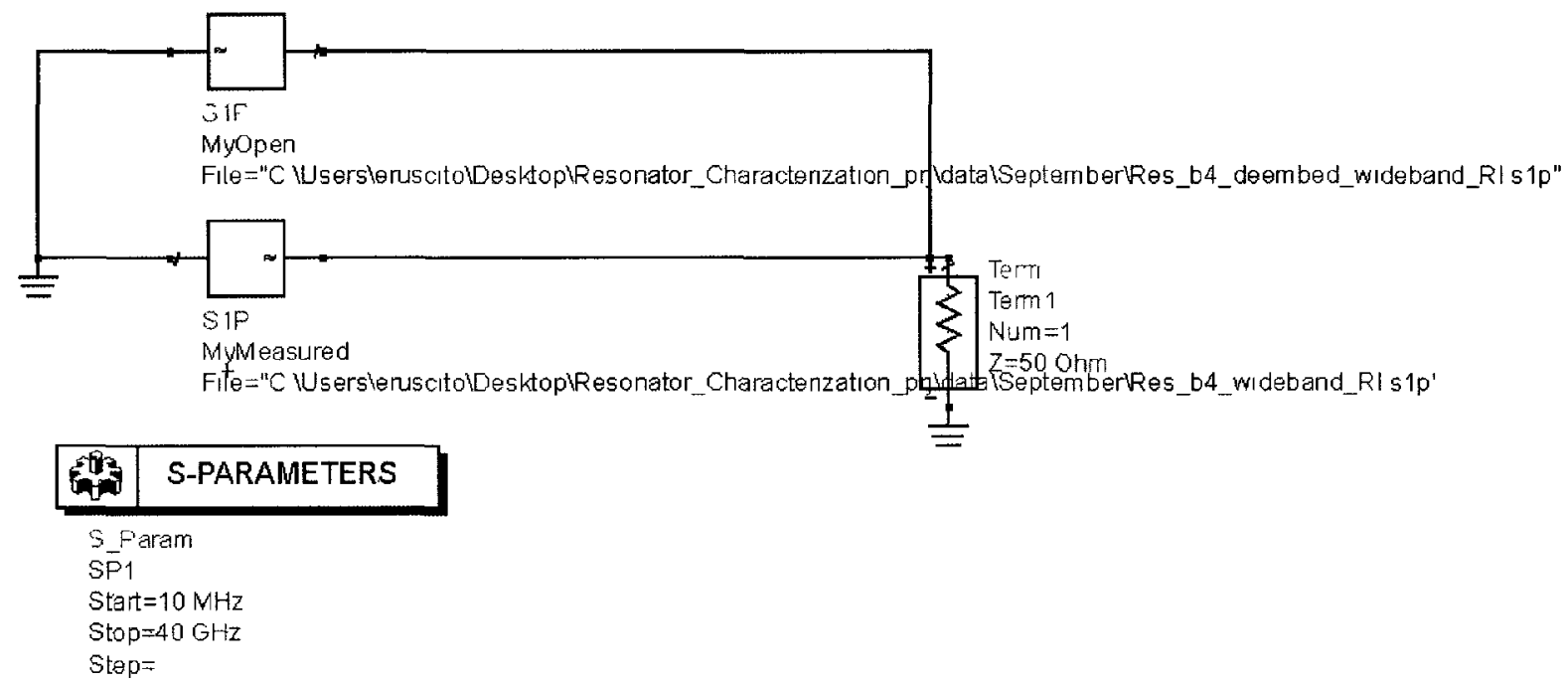

Eqn MyMeasured=stoy(Measured_sept11..S(1,1),50)

Eqn MyOpen=stoy $(S(1,1), 50)$

Eqn MyDUTy=MyMeasured-MyOpen

Eqn MyDUT=ytos(MyDUTy,50) 


\section{References}

[1] K. H. Johansson. (2010). Communication systems of the future. Available: http://www.kth.se/en/ees/omskolan/organisation/centra/access/newsandevents/2.1 1592/communication-systems-of-the-future- 1.56063

[2] J. Wells, "Multigigabit wireless technology at $70 \mathrm{GHz}, 80 \mathrm{GHZ}$ and $90 \mathrm{GHz}$," $R F$ Design, pp. 50-58, 2006.

[3] J. Wells, "E-band Wireless Technology," 2008.

[4] E. C. C. Ng, "Microwave Transceiver Circuit Building Blocks," Graduate Division, Engineering-Electrical Engineering and Computer Sciences, University of California, Berkeley.

[5] J. Esterline, "Phase noise: theory versus practicality," Microwave Journal, vol. 10, 2008.

[6] D. Leeson, "A simple model of feedback oscillator noise spectrum," Proceedings of the IEEE, vol. 54, pp. 329-330, 1966.

[7] H. Chuang, "A low cost and low phase noise oscillator for E-band applications," MASc Thesis, Department of Electronics, Carleton University, Ottawa, Ontario, September 2011.

[8] S. R. McLelland, "Micromachined hemispheroidal cavity resonators," $\mathrm{PhD}$ Disseration, Department of Electronics Carleton University, Ottawa, Ontario, Canada, 2007.

[9] D. M. Pozar, Microwave Engineering. New York, USA: John Wiley \& Sons, Inc., 2005.

[10] A. Chenakin, "Phase Noise Reduction in Microwave Oscillators," Microwave Journal, vol. 52, p. 124, 2009.

[11] P. Patel, "A Better Resonator," Technology Review, 2007.

[12] W. Gautier, "High Q Micro-machined Cavity Resonator Filter in Low-Cost Silicon Technology," 2008.

[13] P. Khanna, "Microwave Oscillators: The state of the Technlogy," Microwave Journal, April 5, 2006.

[14] S. B. Cohn, "Microwave Bandpass Filters Containing High-Q Dielectric Resonators," 1968.

[15] L. Harle and L. Katehi, "A silicon micromachined four-pole linear phase filter," IEEE Trans. Microwave Theory Tech., vol. 52, pp. 1598-1607, 2004. 
[16] N. Faure-Muret, et al., "A high-Q quasi-planar filtering solution for $60 \mathrm{GHz}$ applications," IEEE Trans. Microwave Theory Tech., vol. 15, 2005.

[17] A. Brown, et al., "Microwave and millimeter-wave hight-Q micromachined resonators," International Journal of RF and Microwave Computer-Aided Engineering, vol. 9, pp. 326-337, 1999.

[18] M. Hill, et al., "High-Q micromachied resonant cavities in a K-band diplexer configuration," Microwaves, Antennas and Propagation, IEE Proceedings -, vol. 148, pp. 307-312, 2001.

[19] S. R. McLelland, "Micromachined Hemispheroidal Cavity Resonators," IEEE Trans. Microwave Theory Tech., vol. 56, pp. 982-990, April 2008.

[20] R. F. Harrington, "Time Harmonic Electromagnetic Fields," 1961.

[21] Microwave Enciclopedia. (November 2010). Skin Depth. Available: http:/www.microwaves 101.com/encyclopedia/skindepth.cfm

[22] H. A. Bethe, "Theory of Diffraction by Small Holes," The Physical Review, vol. 66, Oct. 1 and 15, 1944.

[23] N. Marcuvitz, Waveguide Handbook vol. 1st edition. New York: McGraw-Hill, 1951.

[24] R. Collin, Field Theory of Guided Waves vol. 1st ed. New York: McGraw-Hill, 1960.

[25] H. Wheeler, "Coupling Holes Between Resonant Cavities or Waveguides Evaluated in Terms of Volume Ratios," IEEE Trans. Microwave Theory Tech., pp. 231-244, Mar. 1964.

[26] R. Yang and A. Omar, "Analysis of thin inclined rectangular aperture with arbitrary location in rectangular waveguide," IEEE Trans. Microwave Theory Tech., vol. 41, pp. 1461-1463, Aug. 1993.

[27] D. Kreinheder and T. Lingren, "Improved selectivity in cylindrical $\mathrm{TE}_{011}$ filters by $\mathrm{TE}_{211} / \mathrm{TE}_{311}$ mode control," IEEE Trans. Microwave Theory Tech., vol. 82, pp. 1383-1387, Sept. 1982.

[28] D. James, et al., "Aperture coupling between microstrip and resonant cavities," IEEE Trans. Microwave Theory Tech., vol. 25, pp. 392-396, May 1977.

[29] G. Painchaud, "A novel microstrip to cylindrical cavity transition and its application in the design of highly stable $12 \mathrm{GHz}$ GaAs FET oscillators," M.A.Sc. Thesis, University of Ottawa, 1976.

[30] Rogers Corporation. (2011). RT/duroid $® 5870 / 5880$ High Frequency Laminates. Available: $\quad$ http://www.rogerscorp.com/acm/products/10/RT-duroid-5870-58805880LZ-High-Frequency-Laminates.aspx

[31] J. M. Drozd and W. T. Joines, "Determining Q using s-parameter data," IEEE Trans. Microwave Theory Tech., vol. 44, pp. 2123-2127, 1996.

[32] E.-Y. Sun and S.-H. Chao, "Unloaded Q measurements - The Critical-Points Method," IEEE Trans. Microwave Theory Tech., vol. 43, pp. 1983-1986, August 1995.

[33] D. Kajfez, "Q factor measurements, analog and digital," presented at the Workshop on The Q-factor Analysis and Measurement, 2000 Asia-Pacific Microwave Conference, December 2000.

[34] D. Kajfez and E. Hwan, "Q-factor measurement with network analyzer," IEEE Trans. Microwave Theory Tech., vol. 32, pp. 666-670, July 1984. 
[35] R. S. Kwok and J. F. Liang, "Characterization of high-Q resonators for microwave-filter applications," IEEE Trans. Microwave Theory Tech., vol. 47, pp. 111-114, January 1999.

[36] L. H. Chua and D. M. Syahkal, "Accurate and direct characterization of high-Q microwave resonators using one-port measurement," IEEE Trans. Microwave Theory Tech., vol. 51, pp. 978-985, 2003.

[37] S. Shahid, et al., "Reflection type Q-factor measurement using standard least squares methods," IET Microw. Antenna Propag., vol. 5, pp. 426-432, 2011.

[38] K. Leong and J. Mazierska, "Precise measurements for the Q factor of dielectric resonators in the transmission mode -accounting for noise, crosstalk, delay of uncalibrated lines, coupling loss, and coupling reactance," IEEE Trans. Microwave Theory Tech., vol. 50, pp. 2115-2127, 2002.

[39] J. Bray and L. Roy, "Measuring the unloaded, loaded and external quality factors of one- and two-port resonators using scattering-parameter magnitudes at fractional power levels," Microwaves, Antennas and Propagation, IEE Proceedings -, vol. 151, pp. 345-350, August 2004.

[40] D. Kajfez, "Graphical analysis of Q circuits (Correspondence)," IEEE Trans. Microwave Theory Tech., vol. 11, pp. 453-454, September 1963.

[41] D. Kajfez, "Random and systematic uncertainties of reflection-type Q-factor measurement with network analyzer," IEEE Trans. Microwave Theory Tech., vol. 51, pp. 512-519, February 2003.

[42] D. Kajfez, Q factor measurments using MATLAB: Artech House, 2011.

[43] D. Nicholson and H. Lee, "Characterization and modeling of bond wires for highfrequency applications," Microwave Engineering Europe, 2006.

[44] Johanson Manufacturing Corporation. Tuning Elements. Available: http://www.johansonmfg.com/pdf/Tuning-Elements.pdf

GGB Industries Inc. (2011). Calibration Substrates. Available: http://www.ggb.com/calsel.html 